\title{
VARIÂNCIAS DE CONTRASTES DE MÉDIAS NOS ENSAIOS EM PARCELAS SUB-SUBDIVIDIDAS ENVOLVENDO UMA UNIDADE PERDIDA
}

\author{
NORBERTO ANTONIO LAVORENTI \\ Engenheiro Agrônomo
}

Orientador: Prof. Dr. HUMBERTO DE CAMPOS

Dissertação apresentada à Escola
Superior de Agricultura "Luiz de
Queiroz", da Universidade de São
Paulo, para obtenção do título
de Mestre em Agronomia. Área
de Concentração em "Estatística
$\theta$ Experimentação Agronômica".

$P|R A C| C A B A$

Estado de São Paulo - Brasil

Outubro, 1985 
A meus pais (JORGE e LEDA)

pelos ensinamentos e opor-

tunidades na vida

A minha esposa (LEILA) pela compreensão e a meus filhos (TIAGO e DIOGO) DEDICO com amor. 


\section{$A G R A D E C I M E N T O S$}

Ao Professor Doutor e acima de tudo amigo Humberto de Cam pos do Departamento de Matemática e Estatística da ESALQ, pelo irrestrito apoio e segura orientação.

Ao PLANALSUCAR - Programa Nacional de Melhoramento da Ca na-de-Açūcar - pela oportunidade e facilidade para a realização desse curso.

A Seção de Mecanização da Estação Regional Norte do PLANALSUCAR pela cessão dos dados experimentais utilizados neste trabalho.

Aos Demais professores do Departamento de Matemática e Es tatística da ESALQ, pelos ensinamentos, amizade e sugestões apresentadas.

Aos Colegas de curso pela amizade e companheirismo.

A Rosa Maria Alves pela boa vontade na decifração dos meus hieróglifos, para o trabalho de datilografia.

A Todos que de alguma forma colaboraram na concretização deste trabalho. 


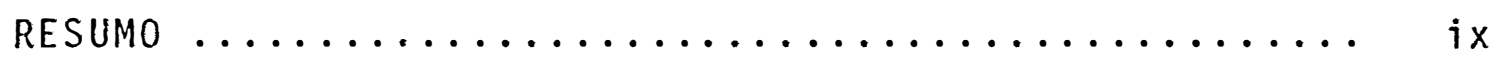
SUMMARY $\ldots \ldots \ldots \ldots \ldots \ldots \ldots \ldots \ldots \ldots \ldots \ldots \ldots \ldots \ldots \ldots \ldots \ldots$

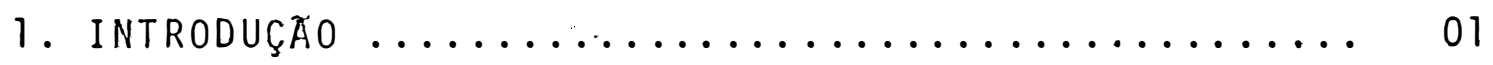

2. REVISATO DE LITERATURA .................... 04

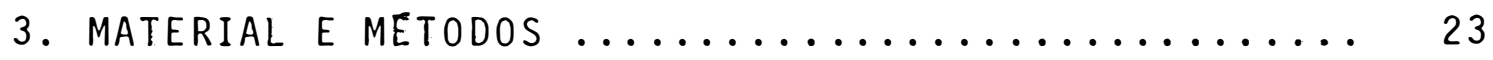

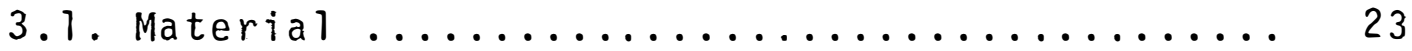

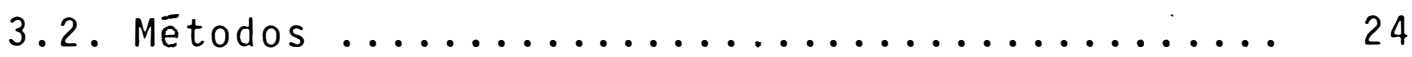

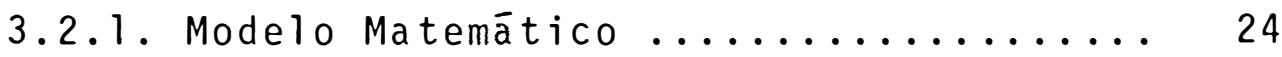

3.2.2. Decomposição da Soma de Quadrado do

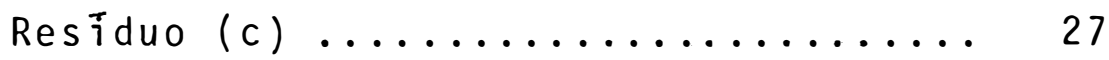

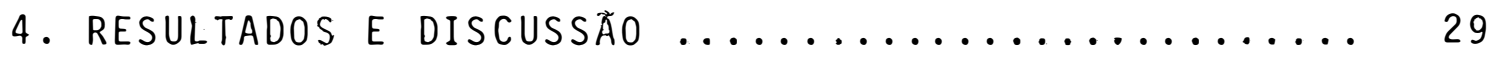

4.1. Decomposição da Soma de Quadrado do Resi-

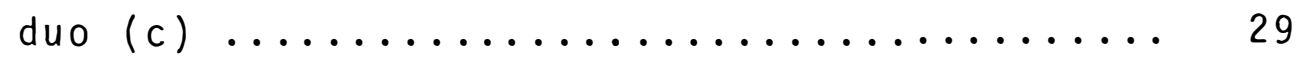

4.2. Esperança Matemātica das Somas de Quadrado dos Quadrados Médios .................. 33

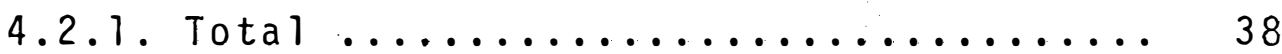

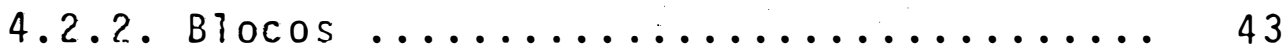

4.2.3. Tratamentos (A) ..................4 47

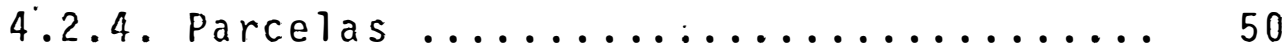

4.2.5. Resíduo (a) ..................... 54

4.2 .6 . Tratamentos (B) ................. 55 
Continuação.

Pàg.

4.2.7. Interacão de Tratamentos ( $A \times B) \ldots \ldots 60$

4.2.8. Subparcelas ................. 64

4.2.9. Residuo (b) ................ 67

4.2.10. Tratamentos (C) ...............6 68

4.2.11. Interação de Tratamentos $(A \times C) \ldots \ldots 72$

4.2.12. Interação de Tratamentos (BxC) .... 76

4.2.13. Interacão de Tratamentos $(A \times B \times C) \ldots 81$

4.2.14. Residuo (c) ................... 85

4.2.15. Exemplo Ilustrativo ............ 88

4.3. Estimadores de Variâncias de Contrastes de Médias Envolvendo o Tratamento com a Sub-Subparcela Perdida ................. 90

4.3.1. Contraste Entre Duas Médias de Tratamentos (A) ................... 90

4.3.2. Contraste Entre Duas Médias de Tratamentos (B) .................... 95

4.3.3. Contraste Entre Duas Médias de Tratamentos (c) .................... 98

4.3.4. Contraste Entre Duas Médias de Tratamentos (B) Dentro do Tratamento

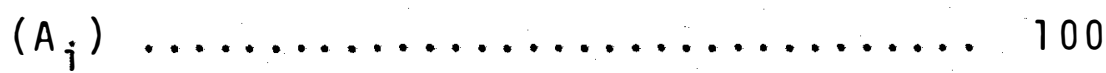


Continuação.

Pāg.

4.3.5. Contraste Entre Duas Médias de Tratá mentos (A) Dentro do Tratamento $\left(B_{k}\right) .104$

4.3.6. Contraste Entre Duas Médias de Trata mentos (C) Dentro do Tratamento $\left(A_{j}\right) .107$

4.3.7. Contraste Entre Duas Médias de Trata mentos (A) Dentro do Tratamento $\left(C_{\ell}\right) .110$

4.3.8. Contraste Entre Duas Médias de Trata mentos (C) Dentro do Tratamento $\left(B_{k}\right)$. 114

4.3.9. Contraste Entre Duas Médias de Trata mentos (B) Dentro do Tratamento $\left(C_{\ell}\right) .117$

4.3.10. Contraste Entre Duas Médias de Trata mentos (C) Dentro dos Tratamentos $\left(A_{j}\right)$ e $\left(B_{k}\right) \ldots \ldots \ldots \ldots \ldots \ldots \ldots \ldots \ldots$

4.3.11. Contraste Entre Duas Médias de Trata mentos (B) Dentro dos Tratamentos $\left(A_{j}\right)$ e $\left(C_{\ell}\right) \ldots \ldots \ldots \ldots \ldots \ldots \ldots \ldots \ldots$

4.3.12. Contraste Entre Duas Médias de Trata mentos (A) Dentrodos Tratamentos $\left(B_{k}\right)$ e $\left(c_{\ell}\right) \ldots \ldots \ldots \ldots \ldots \ldots \ldots$ 
viii.

Continuação.

Pāg.

4.3.13. Exemplo Ilustrativo ........... 130

5. CONCLUSOES .......................... 132

6. LITERATURA CITADA $\ldots \ldots \ldots \ldots \ldots \ldots \ldots \ldots \ldots \ldots \ldots$

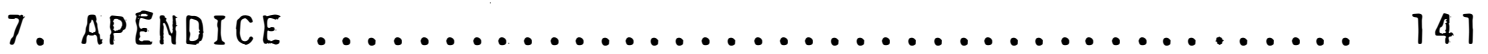


ix.

VARIÂNCIAS DE CONTRASTES DE MEDIAS NOS ENSAIOS EM PAR

CELAS SUB-SUBDIVIVIDAS ENVOLVENDO UMA UNIDADE PERDIDA

Autor: NORBERTO ANTONIO LAVORENTI

Orientador: HUMBERTO DE CAMPOS

R E S U M O

Na experimentação, de um modo geral, quando se têm três fatores para serem estudados simultaneamente, u ma das opções é a utilização do delineamento em parcelas sub-subdivididas. Qualquer que seja o delineamento adotado, o número de unidades experimentais é geralmente elevado, aumentando consequentemente os riscos de perda de uma ou mais unidades experimentais.

No caso de perda de uma ou mais sub-subparce las o valor dessa unidade pode ser estimado e a análise de variância é levada a efeito sem maiores dificuldades. Uma dificuldade surge na estruturação dos estimadores de variâncias de contrastes de médias envolvendo unidades perdidas. 
O objetivo do presente trabalho é a obtenção dos estimadores de variâncias de contrastes envolvendo uma sub-subparcela perdida. Dentre os casos possiveis, foram es tudados os 12 de maior interesse, cujos resultados estão apresentados a seguir:

1. Entre médias de tratamentos primärios A:

$$
\theta\left(\hat{\mathrm{P}}_{i}\right)=\frac{2}{J K L}\left[\mathrm{E}_{\mathrm{a}}+\mathrm{f}(\mathrm{I}-2) \mathrm{E}_{\mathrm{C}}\right] \text {; }
$$

2. Entre médias de tratamentos secundários B:

$$
\hat{\nabla}\left(\hat{Y}_{k}\right)=\frac{2}{I J L}\left[E_{b}+f(K-2) E_{C}\right] ;
$$

3. Entre médias de tratamentos terciários C:

$$
\hat{V}\left(\hat{\mathrm{Y}}_{\ell}\right)=\frac{2 \mathrm{E}_{\mathrm{C}}}{I J K}(1+\mathrm{f} \cdot \mathrm{L}) \quad ;
$$

4. Entre médias de tratamentos B dentro de um mesmo ní vel do tratamento $A: \quad \theta\left(\hat{x}_{K / i}\right)=\frac{2}{J L}\left[E_{b}+f(I K-2) E_{C}\right]$;

5. Entre médias de tratamentos A dentro de um mesmo ní vel do tratamento $\mathrm{B}$ :

$$
\theta\left(\hat{Y}_{i / k}\right)=\frac{2}{J K L}\left[E_{a}+(K-1) E_{b}+f\left(I K^{2}-2 K\right) E_{C}\right] ;
$$

6. Entre médias de tratamentos $\mathrm{C}$ dentro de um mesmo ní vel do tratamento $\left.A: \hat{V}_{\ell / i}\right)=\frac{2 E_{C}}{J K}(1+f \cdot I L)$;

7. Entre médias de tratamentos A dentro de um mesmo ní vel do tratamento $\mathrm{C}$ : 


$$
\hat{v}\left(\hat{Q}_{i / l}\right)=\frac{2 E_{a}}{J K L}+\frac{2 E_{c}}{J K L}\left[(L-1)+f\left(I L^{2}-2\right)\right]
$$

8. Entre médias de tratamentos $C$ dentro de um mesmo ní vel do tratamento $\left.B: \quad V_{\ell / k}\right)=\frac{2 E_{C}}{I J}(I+f \cdot K L)$;

9. Entre médias de tratamentos B dentro de um mesmo ní vel do tratamentos $\mathrm{C}$ :

$$
\nabla\left(\hat{Y}_{k / \ell}\right)=\frac{2 E_{b}}{I J L}+\frac{2 E_{C}}{I J L}\left[(L-1)+f\left(K L^{2}-2\right)\right] ;
$$

10. Entre médias de tratamentos $C$ dentro de mesmo níveis dos tratamentos A e B: $\nabla\left(\hat{P}_{\ell / i k}\right)=\frac{2 E_{C}}{J}(I+f . I K L)$;

11. Entre médias de tratamentos B dentro de mesmo níveis dos tratamentos A e C:

$$
\hat{\theta}\left(\hat{\mathrm{Y}}_{\mathrm{k} / \mathrm{i} \ell}\right)=\frac{2 \mathrm{E}_{\mathrm{b}}}{J L}+\frac{2 \mathrm{E}_{\mathrm{c}}}{\mathrm{JL}}\left[(L-1)+f\left(I K L^{2}-2\right)\right] ;
$$

12. Entre médias de tratamentos A dentro de mesmo níveis dos tratamentos $\mathrm{B}$ e C:

$$
\nabla\left(\hat{Y}_{i / K l}\right)=\frac{2 E_{a}}{J K L}+\frac{2(K-1) E_{b}}{J K L}+\frac{2 E_{c}}{J K L}\left[K(I-1)+f\left(I K^{2} L^{2}-2 K\right)\right],
$$

onde:

$$
\begin{aligned}
& E_{a}=\text { Quadrado médio do resíduo (a); } \\
& E_{b}=\text { Quadrado médio do resíduo (b); } \\
& E_{c}=\text { Quadrado médio do resíduo (c); } \\
& f=\frac{1}{2 I K(J-I)(L-1)}
\end{aligned}
$$


xii.

e ainda:

$$
\begin{aligned}
& I=\text { Número de tratamentos primários }(A) ; \\
& K=\text { Número de tratamentos secundārios }(B) ; \\
& I=\text { Número de tratamentos terciārios }(C), \text { e: } \\
& J=\text { Número de blocos. }
\end{aligned}
$$


VARIANCES OF CONTRASTS AMONG MEANS IN SPLIT-SPLIT-PLOT DESIGNS INVOLVING ONE MISSING SUB-SUB-PLOT

\section{Author: NORBERTO ANTONIO LAVORENTI}

Adviser: HUMBERTO DE CAMPOS

\section{$S U M M A R Y$}

In agricultural research, in general, when three factors have to be studied simultaneously, one option is the using of Split-Split-Plot Designs. Whichever design been adopted, the number of experimental units is generally high, increasing, consequently, the risk of missing one or more experimental units.

In case of missing one or more sub-sub-plots, the experimental unit value can be estimated and the varian ce analysis effectuated whithout large difficulties.

One difficulty appears in the calculus of the variance estimators of the means contrasts involving the missing units.

The objective of this work is to obtain the variance estimators of contrasts involving one missing sub- 
-sub-plot. Among the possible cases, the twelve most interesting were studied. They are:

1. Among means of the primary treatment A:

$$
\theta\left(Q_{i}\right)=\frac{2}{J K L}\left[E_{a}+f(I-2) E_{c}\right]
$$

2. Among means of the secondary treatments $B$ :

$$
\hat{V}\left(\hat{Q}_{\mathrm{k}}\right)=\frac{2}{\mathrm{IJL}}\left[\mathrm{E}_{\mathrm{b}}+\mathrm{f}(\mathrm{K}-2) \mathrm{E}_{\mathrm{C}}\right]
$$

3. Among means of the tertiary treatments $\mathrm{C}$ :

$$
\theta\left(\hat{Q}_{\ell}\right)=\frac{2 E_{C}}{I J K}(I+f \cdot L) \text {; }
$$

4. Among means of the treatments $B$ whithin the same le vel of treatment $A: \quad \theta\left(\Theta_{k / i}\right)=\frac{2}{J L}\left[E_{b}+f(I K-2) E_{c}\right]$;

5. Among means of the treatments $A$ whithin the same level of treatment $B$ :

$$
\hat{\nabla}\left(\hat{Y}_{i / k}\right)=\frac{2}{J K L}\left[E_{a}+(K-I) E_{b}+f\left(I K^{2}-2 K\right) E_{c}\right] ;
$$

6. Among means of the treatments $C$ whithin the same level of treatment $A: \quad \theta\left(\hat{Y}_{l / i}\right)=\frac{2 E_{C}}{J K}(1+f$. IL);

7. Among means of the treatments $A$ whithin the same level of treatment $\mathrm{C}$ :

$$
\theta\left(\hat{Y}_{i / \ell}\right)=\frac{2 E_{a}}{J K L}+\frac{2 E_{c}}{J K L}\left[(I-1)+f\left(I L^{2}-2\right)\right] ;
$$


8. Among means of the treatments $C$ whithin the same level of treatment $B: \quad \theta\left(\hat{Y}_{\ell / k}\right)=\frac{2 E_{C}}{I J}(1+f \cdot K L)$;

9. Among means of the treatments $B$ whithin the same level of treatment $\mathrm{C}$ :

$$
\theta\left(\hat{Y}_{k / \ell}\right)=\frac{2 E_{b}}{I J L}+\frac{2 E_{c}}{I J L}\left[(L-I)+f\left(K L^{2}-2\right)\right] ;
$$

10. Among means of the treatments $C$ whithin the same levels of treatments $A$ and $B$ :

$$
\theta\left(\hat{Y}_{\ell / i k}\right)=\frac{2 E_{C}}{J}(I+f \cdot I K L) ;
$$

11. Among means of the treatments $B$ whithin the same levels of treatments $A$ and $C$ :

$$
\theta\left(Q_{k / 1 l}\right)=\frac{2 E_{b}}{J L}+\frac{2 E_{C}}{J L}\left[(L-1)+f\left(I K L^{2}-2\right)\right] ; \text { and }
$$

12. Among means of the treatments $A$ whithin the same levels of treatments $B$ and $C$ :

$$
\theta\left(\hat{Y}_{i / K l}\right)=\frac{2 E_{a}}{\pi L}+\frac{2(K-1) E_{b}}{J K L}+\frac{2 E_{c}}{\pi K L}\left[K(I-1)+f\left(I K^{2} I^{2}-2 K\right)\right]
$$

where:

$$
\begin{aligned}
& E_{a}=\text { Error (a) mean square; } \\
& E_{b}=\text { Error (b) mean square; } \\
& E_{c}=\text { Error (c) mean square; } \\
& f=\frac{1}{2 \operatorname{IK}(\mathrm{J}-\mathrm{l})(\mathrm{L}-\mathrm{l})}
\end{aligned}
$$


xvi.

and:

$$
\begin{aligned}
& I=\text { Number of primary treatments }(A) ; \\
& K=\text { Number of secondary treatments }(B) ; \\
& L=\text { Number of tertiary treatments }(C), \text { and: } \\
& \mathrm{J}=\text { Number of blocks. }
\end{aligned}
$$


1. INTRODUÇAOO

Os ensaios em parcelas sub-subdivididas são maito üteis para se estudar três fatores quando, por circunstân cias diversas, de execução por exemplo, não se pode instalar o experimento num esquema fatorial.

Com três fatores sendo estudados concomitantemente, 0 número de unidades experimentais aumenta consideravelmente, e em igual proporção os riscos de perdas de ob servações devido as mais diversas causas, alheias ou não ao controle do experimentador.

As vezes não há propriamente perda da observação, mas o valor encontrado, representativo daquela sub-sub-unidade, discrepa consideravelmente dos demais valores do ensaio ou não é fidedigno. Nesses casos é recomendável ad mitir a perda da observação. 
Não raro o número de observações perdidas é tanto que se for utilizado o processo usual de estimar os seus valores, o ensaio ficará de tal forma artificial, que comprometerá a validade das conclusões obtidas.

Quando se estimam unidades experimentais, 0 nümero de graus de liberdade do residuo correspondente, seja ao nivel de subparcelas ou de sub-subparcelas, fica reduzido de 1 grau de liberdade por unidade estimada, pois essas estí mativas não têm erro experimental.

O procedimento de recuperar informações perdidas faz com que algumas somas de quadrados nas análises de variância fiquem alteradas, o que, conforme o caso, pode pre judicar as conclusões finais.

De forma prática, numa análise de variância quando se têm valores de $\mathrm{F}$ altamente significativos dispensa-se a correção da soma de quadrados. No caso de valores de F próximos ao limite de significância, a introdução da cor reção poderá modificâr as conclusões preliminares obtidas.

A maior dificuldade no caso de observações perdidas nos experimentos em parcelas sub-subdivididas se situa no emprego dos métodos de comparações múltiplas, onde é notó ria a multiplicidade de casos, correspondendo a cada um deles um distinto estimador de variância de contraste entre mê dias. 
O objetivo do presente trabalho é a obtenção dos estimadores de variâncias de contrastes, envolvendo uma observação perdida, nos casos de maior interesse prático. 
4.

\section{REVISATO DE LITERATURA}

Um dos primeiros trabalhos a respeito de per da de parcelas foi apresentado por ALLAN e WISHART (1930) no qual os autores estjmavam seus valores através de um pro cesso que, a princípio, pensou-se tratar do Método dos Míni mos Quadrados. Através de cálculo diferencial e aplicando certos artifícios esses autores conseguiram fórmulas que es timavam parcelas perdidas para os delineamentos em blocos casualizados e em quadrados latinos.

YATES (1933) utilizando incógnitas para subs tituir os valores das parcelas perdidas e aplicando o Método dos Mínimos Quadrados, chegou aos mesmos resultados apre sentados por ALLAN e WISHART (1930). 
Um trabalho bem mais completo foi realizado posteriormente por ANDERSON (1946) no qual são apresentadas fórmulas para o cálculo de parcelas perdidas nos mais dife rentes delineamentos, inclusive para o de parcelas subdivididas. Segundo o autor, o melhor estimador da parcela perdi da, e que torna mínimo o erro experimental a nível de subpar cela, è:

$$
Y=\frac{r\left(R_{j} A_{i}\right)+B\left(A_{i} B_{k}\right)-A_{i}}{(r-I)(B-1)}
$$

onde:

$$
\begin{aligned}
Y & \text { é a estimativa da subparcela perdida; } \\
A_{i}= & \text { total de todas as subparcelas com o tratamento } i ; \\
R_{j} A_{i}= & \text { total de todas as subparcelas com o tratamento i } \\
& \text { na repetição } j ; \\
A_{i} B_{k}= & \text { total de todas as subparcelas existentes envolven } \\
& \text { do a combinação dos tratamentos } i \mathrm{e} ; \\
r= & \text { número de repetições, e: } \\
B= & \text { número de tratamentos secundários. }
\end{aligned}
$$

Neste trabalho o autor afirma que para a ob tenção das somas de quadrados não há necessidade de se preo cupar com as observações perdidas. 
Assim, para o cálculo das variâncias das dị ferenças entre médias de tratamentos secundários ele apresenta as seguintes fórmulas:

(1) $\quad \overline{\mathrm{B}}_{\mathrm{k}}^{*}-\overline{\mathrm{B}}_{\mathrm{k}}, \quad \Longrightarrow \frac{2 \cdot \mathrm{E}_{\mathrm{b}}}{\mathrm{ra}-1}$

(2) $\overline{A_{i} B_{k}^{K}}-\overline{A_{i} B_{k}^{\prime}} \Longrightarrow \frac{2 \cdot E_{b}}{r-1}$

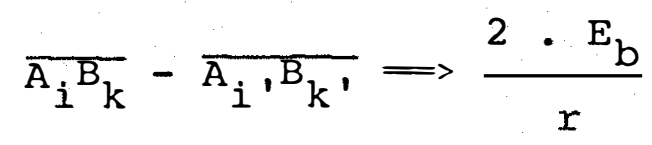

$$
\begin{aligned}
& \overline{A_{i} B_{k}^{*}}-\overline{A_{i}{ }^{\prime} B_{k}} \Longrightarrow\left[\frac{E_{a}+(\beta-1) \cdot E_{b}}{\beta}\right]\left[\frac{1}{r}+\frac{r}{r-1}\right] \\
& \overline{A_{i}{ }^{\prime} B_{k}}-\overline{A_{i}{ }^{\prime} B_{k}} \Longrightarrow \frac{2}{r}\left[\frac{E_{a}+(\beta-1) \cdot E_{b}}{\beta}\right]
\end{aligned}
$$

onde:

a é o número de tratamentos primários;

$\mathrm{E}_{\mathrm{a}}$ é a variância residual a nível de parcelas, e:

$E_{b}$ é a variância residual a nível de subparcelas;

* casos em que houve perda de uma subparcela

Afirma ainda que se o valor estimado $Y$ for utilizado na análise, todas as somas de quadrados ficarão ligeiramente superestimadas com exceção da S.Q. Resíduo (b) e que o teste de significância para os tratamentos a nível 
7.

de parcelas não fica seriamente afetado, devido à pequena tendenciosidade introduzida pelo uso do valor estimado da $\underline{\text { u}}$ nidade perdida.

Os autores TRUITT e SMITH (1956) fazem men ção ao ajuste feito através do uso de covariância e de variāveis "dumny", e citam ainda as metodologias empregadas por ANDERSON e BARTLETT, O mesmo ocorrendo com os autores JOHN e QUENOUILLE (1977) que colocam essa técnica como a mais apropriada.

Fórmulas mais genéricas de estimativas das variâncias de contrastes entre duas médias, para ensaios em parcelas subdivididas, foram introduzidas por COCHRAN e COX (1957), e são apresentadas a seguir:

\begin{tabular}{ll} 
Contraste & Variância \\
\hline$a_{i}-a_{i}$, & $\frac{2 \cdot\left(E_{a}+f \cdot E_{b}\right)}{r b}$ \\
$b_{j}-b_{j}$, & $\frac{2 \cdot E_{b}(1+f \cdot b / a)}{r a}$ \\
$a_{i} b_{j}-a_{i} b_{j}$, & $\frac{2 \cdot E_{b}(1+f \cdot b / a)}{r}$ \\
$a_{i} b_{j}-a_{i} \cdot b_{j}$ ou & $\frac{2 \cdot E_{a}+2 \cdot E_{b}\left[(b-1)+f \cdot b^{2}\right]}{r b}$ \\
$a_{i} b_{j}-a_{i} \cdot b_{j}$, & $\frac{r}{}$
\end{tabular}


onde:

$E_{a}$ é o quadrado médio do resíduo (a);

$\mathrm{E}_{\mathrm{b}}$ è o quadrado médio do resíduo (b) :

b é o número de tratamentos secundários;

a é o número de tratamentos primários, e:

r è o número de repetições.

o fator f possui as seguintes característi-

cas :

$$
f=\frac{1}{2(x-1)(b-1)},
$$

para os casos em que somente uma subparcela foi perdida, e:

$$
f=\frac{k}{2(r-d)(b-k+e-1)}
$$

para os casos de mais de uma subparcela perdida e está na dependência da localização dessas perdas, onde:

$k$ é o número de observações perdidas;

e é o número de blocos que contém uma ou mais observações perdidas, e:

d é o número de observações perididas na unidade da combinação dos tratamentos $a_{i} b_{j}$ que é mais afetada. 
Esses autores citam ainda o caso de mais de uma subdivisão das parcelas, isto é, o caso de sub-subparce las, mas sem considerarem observaçōes perdidas. Apresentam os erros padrão das diferenças entre duas médias, conforme se verifica a seguir:

Comparações de Médias

Erros-padrão

$a_{i}-a_{i}$

$\sqrt{\frac{2 \cdot E_{a}}{r \cdot b \cdot c}}$

$b_{j}-b_{j}$

$\sqrt{\frac{2 \cdot \mathrm{E}_{\mathrm{b}}}{\mathrm{r} \cdot \mathrm{a} \cdot \mathrm{c}}}$

$a_{i} b_{j}-a_{i} b_{j}$

$\sqrt{\frac{2 \cdot E_{b}}{r \cdot c}}$

$\left[a_{i} b_{j}-a_{i}, b_{j}\right]$ ou $\left[a_{i} b_{j}-a_{i}, b_{j}\right]$

$\sqrt{\frac{2\left[\overline{\left.(b-1) E_{b}+E_{a}\right]}\right.}{r \cdot b \cdot c}}$

$c_{k}-c_{k}$

$\sqrt{\frac{2 \cdot E_{C}}{\text { r.a.b }}}$

$a_{i} c_{k}-a_{i} c_{k}$

$\sqrt{\frac{2 \cdot E_{c}}{r \cdot b}}$

$b_{j} c_{k}-b_{j} c_{k}$

$\sqrt{\frac{2 \cdot E_{c}}{I \cdot a}}$

$a_{i} b_{j} c_{k}-a_{i} b_{j} c_{k}$,

$\sqrt{\frac{2 \cdot E_{c}}{I}}$ 
Continuaçäo.

Comparaçöes de Médias Erros-padrão

$$
\begin{aligned}
& {\left[b_{j} c_{k}-b_{j}, c_{k}\right] \text { ou }\left[b_{j} c_{k}-b_{j}, c_{k^{\prime}}\right] \quad \sqrt{\frac{2\left[(c-1) E_{c}+E_{b}\right]}{r \cdot a \cdot c}}} \\
& a_{i} b_{j} c_{k}-a_{i} b_{j} \cdot c_{k} \\
& \sqrt{\frac{2\left[(c-1) E_{c}+E_{b}\right]}{r \cdot c}} \\
& {\left[a_{i} c_{k}-a_{i}, c_{k}\right] \text { ou }\left[a_{i} c_{k}-a_{i}, c_{k}\right] \quad \sqrt{\frac{2\left[(c-1) E_{c}+E_{a}\right]}{r \cdot b \cdot c}}} \\
& a_{i} b_{j} c_{k}-a_{i} \cdot b_{j} c_{k} \\
& \sqrt{\frac{2\left[b(c-1) E_{c}+(b-1) E_{b}+E_{a}\right]}{r \cdot b \cdot c}}
\end{aligned}
$$

onde:

$$
\begin{aligned}
& \text { c é o número de tṛatamentos terciários, e: } \\
& \text { E }_{C} \text { è o quadrado médio do resíduo (c). }
\end{aligned}
$$

CAMPOS (1964) apresenta um estudo bastante in teressante sobre o cálculo de parcelas perdidas, utilizando em seu trabalho o Método Iterativo ou das aproximações suces sivas na obtençäo de estimativas de duas ou mais parcelas per didas nos delineamentos em blocos casualizados e em quadrado latino, estendendo esse conceito para os ensaios em períodos sucessivos. 
Fórmulas para obtenção da estimativa de uma e de duas sub-subparcelas foram apresentadas por PINHO (1973), englobando todos os casos possíveis de perdas. Para o caso de uma observaçäo perdida a sua estimativa pode ser obtida a través da equação:

$$
y_{1}=\frac{r(S P)+c\left(A_{j} B_{k} C_{l}\right)-A_{j} B_{k}}{(r-1)(c-1)}
$$

onde:

$$
\begin{aligned}
& y_{1} \text { é a estimativa da unidade perdida; } \\
& \text { r é o número de blocos ; } \\
& \text { c é o nümero de tratamentos terciários; } \\
& \text { SP é o total das sub-subparcelas presentes na subpar }
\end{aligned}
$$

A autora apresenta ainda a análise de variân cia, com as equações para obtenção das somas de quadrados, bem como suas respectivas correções, que estão assim estrutú radas : 


$$
\text { S.O.Blocos }=\frac{1}{a b c}\left[\left(y_{1}+B L\right)^{2}+D\right]-C
$$

A correção correspondente é:

$$
\begin{aligned}
\mathrm{U}_{\mathrm{Blocos}}=\frac{(r-1)}{\mathrm{rabc}} \mathrm{y}_{1}^{2} & +2 \mathrm{y}_{1} \frac{\mathrm{rBL}-\mathrm{G}}{\mathrm{rabc}}-\frac{\mathrm{a}(\mathrm{rbc}-1)-(\mathrm{r}-1)}{\mathrm{rabc}} \mathrm{y}_{9}^{2}+ \\
& +\frac{\mathrm{rbc}-1}{\mathrm{rbc}} \mathrm{y}_{11}^{2}
\end{aligned}
$$

onde:

$$
\begin{aligned}
& \text { a é o número de tratamentos primários A; } \\
& \text { b é o número de tratamentos secundários B; } \\
& \text { BL é o total das unidades restantes no bloco da sub- } \\
& \text {-subparcela perdida ; } \\
& \text { D é a soma de quadrados dos totais de cada bloco ex } \\
& \text { cetuando o da sub-subparcela perdida; } \\
& \text { C é a correção dada por } \frac{\left(\underline{y}_{1}+G\right)^{2}}{r a b c} \text {; } \\
& G \text { é o total das sub-subparcelas restantes; } \\
& y_{9}=\frac{a_{j}+r B L-G}{(r a b c-a-r+1)} \text {; } \\
& A_{j} \text { é o total de todas as unidades relativas ao j-és } \\
& \text { mo tratamento A atribuido à parcela com a unidade } \\
& \text { perdida, e: }
\end{aligned}
$$


13.

$$
y_{11}=\frac{A_{j}}{r b c-1} \text {. }
$$

$$
\text { S.Q. Tratamentos }(A)=\frac{1}{\operatorname{rbc}}\left[\left(y_{1}+A_{j}\right)^{2}+E\right]-C
$$

com a correção:

$$
\begin{aligned}
U_{\mathrm{TA}}=\frac{a-1}{\mathrm{rabc} y_{1}^{2}} & +2 y_{1} \frac{a A_{j}-G}{r a b c}-\frac{a(r b c-1)-(r-1)}{r a b c} y_{9}^{2}+ \\
& +\frac{a b c-1}{a b c} y_{10}^{2}
\end{aligned}
$$

onde:

E é a soma dos quadrados dos totais de cada nível do tratamento $A$, exceto o relativo à sub-subparcela perdida

$$
y_{10}=\frac{B L}{a b c-1}
$$

$$
\text { S.Q.Parcelas }=\frac{1}{b c}\left[\left(y_{1}+P\right)^{2}+F\right]-C
$$

onde:

$P$ è o total das sub-subparcelas presentes na parcela onde se perdeu uma unidade ;

F é a soma de quadrados de cada parcela, excetuando-se o da observaçao perdida. 


$$
\begin{aligned}
\text { S.Q.Resíduo }(a)= & \text { S.Q.Parcelas - S.Q.Blocos - S.Q.Tra } \\
& \text { tamentos }(A) .
\end{aligned}
$$

A expressão da correção associada a este resíduo è:

$$
\begin{gathered}
U_{R A}=\frac{(a-1)(r-1)}{r a b c} y_{1}^{2}+2 y_{1} \frac{r a P-a A_{j}-r B L+G}{r a b c}-\frac{b c-1}{b c} y_{8}^{2}+ \\
+\frac{a(r b c-1)-(r-1)}{r a b c} y_{9}^{2}
\end{gathered}
$$

onde:

$$
y_{8}=\frac{P}{b c-1}
$$

$$
\text { S.Q.Tratamentos }(B)=\frac{1}{\operatorname{rac}}\left[\left(y_{1}+B_{k}\right)^{2}+H\right]-C
$$

e a correção:

$$
\begin{aligned}
U_{T B}=\frac{b-1}{r a b c} y_{1}^{2} & +2 y_{1} \frac{b B_{k}-G}{r a b c}-\frac{r a(b c-1)-(b-1)}{r a b c} y_{7}^{2}+ \\
& +\frac{b c-1}{b c} y_{8}^{2}
\end{aligned}
$$

onde:

$$
\begin{array}{r}
B_{k} \text { é o total de todas as observações relativas ao } \\
\text { k-ēsimo tratamento } B \text { atribuído à unidade perdida }
\end{array}
$$


H è a soma de quadrados dos totais de cada tratamen to $B$, excetuando-se o da sub-subparcela perdida, e

$$
y_{7}=\frac{r a P+b B_{k}-G}{(r a b c-r a-b+1)}
$$

(6) S.Q. Tratamentos $(A \times B)=\frac{1}{r C}\left[\left(y_{l}+A_{j} B_{k}\right)^{2}+M\right]-C-$

$$
\text { - S. ..Trat. (A) - S. O.Trat. (B) }
$$

e a correção:

$$
\begin{aligned}
U_{A B}= & \frac{(a-1)(b-1)}{r a b c} y_{1}^{2}+2 y_{1} \frac{a b\left(A_{j} B_{k}\right)-a A_{j}-b B_{k}+G}{r a b c}- \\
& -\frac{b(r c-1)-(r-1)}{r b c} y_{6}^{2}+\frac{r a(b c-1)-(b-1)}{r a b c} y_{7}^{2}
\end{aligned}
$$

onde:

M é a soma dos quadrados dos totais de cada combinação $A_{j} B_{k}$, excetuando-se o da sub-subparcela per dida, e

$$
y_{6}=\frac{r P+b\left(A_{j} B_{k}\right)-A_{j}}{(r b c-r-b+1)} .
$$

$$
\text { S.Q.Subparcelas }=\frac{1}{C}\left[\left(y_{1}+S P\right)^{2}+N\right]-C
$$


onde:

$\mathrm{N}$ é a soma de quadrados dos totais de cada subparcela, excetuando-se o da sub-subparcela perdida.

(8)

$$
\begin{aligned}
\text { S. . Resíduo (b) = } & \text { S.Q.Subparcelas - S.Q.Parcelas - } \\
& \text { - S.O.Tratamentos (B)-S.Q.Tratamen } \\
& \text { tos (AxB) }
\end{aligned}
$$

com a correção sendo expressa por:

$$
\begin{aligned}
U_{R A B}= & \frac{(b-1)(r-1)}{r b c} y_{1}^{2}+2 y_{1} \frac{r b(S P)-b\left(A_{j} B_{k}\right)-r P+A_{j}}{r b c}- \\
& -\frac{c-1}{c} y_{5}^{2}+\frac{b(r c-1)-(r-1)}{r b c} y_{6}^{2}
\end{aligned}
$$

com:

$$
y_{5}=\frac{S P}{c}-\frac{S}{1}
$$

$$
\text { S.Q. Tratamentos̀ }(C)=\frac{1}{\operatorname{rab}}\left[\left(y_{1}+C_{\ell}\right)^{2}+0\right]-C
$$

e a correção:

$$
U_{T C}=\frac{c-1}{r a b c} Y_{I}^{2}+2 Y_{1} \frac{c c_{\ell}-G}{r a b c}-\frac{(c-1)(r a b-1)}{r a b c} Y_{4}^{2}+\frac{c-1}{c} y_{5}^{2}
$$


onde:

$c_{\ell}$ é o total de todas as observações relativas ao l-ésimo tratamento $\mathrm{C}$ atribuído à unidade perdida;

Q é a soma dos quadrados dos totais de cada tratamento $C$, excetuando-se o da sub-subparcela perdi da, e:

$y_{4}=\frac{r a b(S P)+c C_{\ell}-G}{(c-1)(r a b-1)}$

(10) S.Q.Tratamentos $(A x C)=\frac{1}{r b}\left[\left(y_{1}+A_{j} C_{l}\right)^{2}+R\right]-C-$

- S.Q.Tratamentos (A) -

- S.Q.Tratamentos (C)

com:

$$
\begin{aligned}
U_{A C}= & \frac{(a-1)(c-1)}{r a b c} y_{1}^{2}+2 y_{1} \frac{a c\left(A_{j} c_{l}\right)-a A_{j}-c c_{\ell}+G}{r a b c}- \\
& -\frac{(c-1)(r b-1)}{r b c} y_{3}^{2}+\frac{(c-1)(r a b-1)}{r a b c} y_{4}^{2}
\end{aligned}
$$

$A_{j} C_{\ell} \bar{e} \circ$ total de todas as observaçöes relativas à $A_{j}$ e $c_{\ell}$, correspondentes à sub-subparcela perdida;

$R$ é a soma dos quadrados dos totais de cada nível de combinaçao $A_{j} C_{l}$, excetuando-se $\circ$ da sub-subparcela perdida, e : 
18.

$$
y_{3}=\frac{r b(S P)+c\left(A_{j} C_{l}\right)-A_{j}}{(c-1)(r b-1)} .
$$

$$
\text { S.2.Tratamentos }(\mathrm{BxC})=\frac{1}{\mathrm{ra}}\left[\left(\mathrm{y}_{1}+\mathrm{B}_{\mathrm{k}} \mathrm{C}\right)^{2}+\mathrm{s}\right]-\mathrm{C}-
$$

- S. . Tratamentos (B) - S.@.Tratamentos

com:

$$
\begin{aligned}
U_{B C}= & \frac{(b-1)(c-1)}{r a b c} y_{1}^{2}+2 y_{1} \frac{b c\left(B_{k} C_{l}\right)-b B_{k}-c_{l}+G}{r a b c}- \\
& -\frac{(c-1)(r a b-b-a+1)}{r a b c} y_{2}^{2}+\frac{(c-1)(r b-1)}{r b c}-y_{3}^{2}
\end{aligned}
$$

onde :

$\mathrm{B}_{k} \mathrm{C}_{\ell}$ é o total de todas as observações relativas à combinaçăo $\mathrm{B}_{k}$ e $\mathrm{C}_{\ell}$, correspondentes à sub-sub parcela perdida;

S é a soma dos quadrados dos totais de cada nível da combinação $B_{k} C_{\ell}$, excetuando-se aquele da sub-subparcela perdida, e:

$$
y_{2}=\frac{r a b(S P)+b c\left(B_{k} C_{\ell}\right)+a c\left(A_{j} C_{\ell}\right)-a A_{j}-b B_{k}-c C_{\ell}+G}{(c-1)(r a b-b-a+1)}
$$


(12) S.Q.Tratamentos $(A \times B \times C)=\frac{1}{r}\left[\left(y_{1}+A_{j} B_{k} C_{l}\right)^{2}+v\right]-C-$ - S.Q.Trat. (A) - S.Q.Trat. (B) -

- S.Q.Trat. (AXB) - S.Q.Trat. (AXC) -

- S.Q.Trat. (BXC) - S.Q.Trat. (C)

com a expressão de correção sendo dada por:

$$
\mathrm{U}_{\mathrm{ABC}}=\frac{(\mathrm{c}-\mathrm{l})(\mathrm{rab}-\mathrm{a}-\mathrm{b}+1)}{\mathrm{rabc}}\left(\mathrm{y}_{1}-\mathrm{y}_{2}\right)^{2}
$$

onde :

$\mathrm{V}$ é a soma dos quadrados dos totais de cada nível da combinação $\mathrm{A}_{j} \mathrm{~B}_{k} \mathrm{C}_{l}$, excetuando-se o da sub-subparcela perdida.

(i3) S.Q.Total $=\mathrm{y}_{1}^{2}+\mathrm{w}-\mathrm{C}$

onde:

W é a soma dos quadrados de cada sub-subparcela pre sente no ensaio. 
e neste caso não existe a correção dos casos anteriores.

Assim, conclui PINHO (1973), a correção U $\mathrm{ABC}$ será sempre positiva, implicando numa s.Q.tratamentos (AXBXC) sempre superestimada, enquanto que as demais correções podem assumir valores tanto positivos quanto negativos, depen dendo dos valores estimados para a sub-subparcela.

PINHO (1975) apresentou um estudo sobre com ponentes de variância nos ensaios em parcelas subdivididas com uma observação perdida. Neste trabalho, determinou os estimadores das variâncias dos diversos contrastes, envolvendo a média de tratamento com observação perdida e compa rou esses estimadores com os obtidos por ANDERSON (1946), COCHRAN e COX (1957) e STEEL e TORRIE (1960). Seus resultados foram:

(1) Para duas médias de tratamentos $\mathrm{T}$ :

$$
\hat{V}\left(\hat{Y}_{i}\right)=\frac{2}{J K}\left[E_{a}+\frac{(I-2) E_{b}}{2 I(J-I)(K-I)}\right]
$$


onde:

$$
\begin{aligned}
& J \text { é o número de blocos ; } \\
& \text { I é o número de tratamentos primários (T); } \\
& K \text { é o número de tratamentos secundários (T'); } \\
& E_{a} \text { é o quadradó médio do resíduo (a), e: } \\
& E_{b} \text { é o quadrado médio do resídio (b). }
\end{aligned}
$$

(2) Para duas médias de tratamentos T':

$$
\hat{V}\left(\hat{Y}_{k}\right)=\frac{2 E_{b}}{I J}\left[I+\frac{K}{2 I(J-I)(K-1)}\right]
$$

(3) Para duas médias de $\mathrm{T}^{\prime}$ no mesmo nível de $\mathrm{T}$ :

$$
\hat{V}\left(\hat{Y}_{k / i}\right)=\frac{2 E_{b}}{J}\left[1+\frac{K}{2(J-1)(K-I)}\right]
$$

(4) Para duas médias de $\mathrm{T}$ no mesmo nível de T':

$$
\hat{V}\left(\hat{Y}_{i / K}\right)=\frac{2 E_{a}}{J K}+\frac{2 E_{b}}{J K}\left[(K-1)+\frac{I K^{2}-2}{2 I(J-I)(K-I)}\right]
$$

GOMES (1976) apresenta também fórmula para a estimativa de uma parcela perdida nos delineamentos em parcelas subdivididas, que é concordante com a citada por COCHRAN e COX (1957). 
LITTLE e HILLS (1977) apresentam os mesmos resultados de COCHRAN e COX (1957) e estabelecem relações pa ra a aplicação do teste " $t$ " no confronto de médias de tratạ mentos $(a, b, c)$.

Variâncias de contrastes entre efeitos de tratamentos são mostradas por SANCHES (1978) para os casos com e sem parcelas perdidas nos ensaios em quadrado latino. HADDAD (1978) apresentou um caso de perda de uma parcela nos delineamentos em parcelas subdivididas, com $K$ subparcelas e nos casos em que $K \geq 2$. Demonstrou que a soma de quadrados de subtratamentos ajustada é igual àque la obtida usualmente.

SANCHES (1980) complementou o trabalho de HADDAD (1978) deduzindo, além das fórmulas que estimam as perdas, a anālise de variância. 
23.

3. MATERIAL E METODOS

3.1. Material

Com o objetivo de ilustrar os resultados ob tidos no desenvolvimento teórico deste trabalho serão utili zados dados referentes à determinação do "DEW-LAP" de col mos de cana-de-açūcar de um ensaio sobre a melhor combinação entre espaçamentos, variedades e densidades de gemas no plantio. O experimenţo foi conduzido pela Seção de Mecaniza ção da Estação Regional do IAA/PLANALSUCAR e instalado na Destilaria UBU, em Pernambuco. Nesse experimento foi utilizado o delineamento em parcelas sub-subdivididas, com 4 es

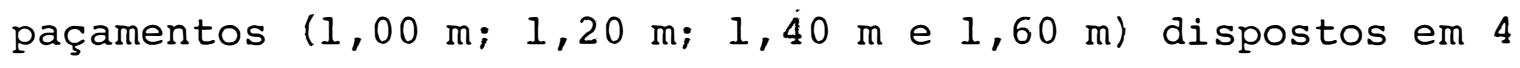
blocos, constituindo as parcelas. Nas subparcelas foram uti 
lizadas 3 variedades de cana-de-açúcar (CB 45-3, CO 997 e RB 72454) e, a nível de sub-subparcelas, 3 densidades de ge. mas no plantio ( 12 gemas $/ \mathrm{m} ; 15$ gemas $/ \mathrm{m}$ e 18 gemas $/ \mathrm{m})$.

\subsection{Métodos}

\subsubsection{Modeio Matemático}

Considerando o delineamento em parcelas sub-subdivididas com I tratamentos primários dispostos em .J blocos, $K$ tratamentos secundários e I tratamentos terciários, o modelo matemático apropriado ao caso é:

$$
\begin{aligned}
x_{i j k \ell}=m & +a_{i}+r_{j}+(a r)_{i j}+b_{k}+(a b)_{i k}+(a b r)_{i k j}+ \\
& +c_{\ell}+(a c)_{i \ell}+(b c)_{k \ell}+(a b c)_{i k \ell}+e_{i j k \ell}
\end{aligned}
$$

onde:

$$
\begin{aligned}
& x_{i j k \ell} \text { é a j-ésima observação da combinação dos tratạ } \\
& \text { mentos } a_{i} b_{k} c_{l} \\
& \text { m é a média geral ; } \\
& a_{i} \text { é o efeito do tratamento primário i, com } \\
& i=1,2, \ldots, I_{i} \text {. } \\
& r_{j} \text { é o efeito do bloco } j, \operatorname{com} j=1,2, \ldots, J_{i} \\
& (a r)_{i j} \text { é o erro residual a nível de parcelas, admiti- }
\end{aligned}
$$


$b_{k}$ é o efeito do tratamento secundário k, com $\mathrm{k}=1,2, \ldots, \mathrm{k}$;

(ab) ik é o efeito da interação do tratamento primário i com o tratamento secundário $k$;

$(a b r)_{i k j}$ é o erro residual a nível de subparcelas, tam bém admitido aleatório;

$c_{\ell}$ é o efeito do tratamento terciário $\ell$, com $\ell=1,2, \ldots, L$;

(ac) ${ }_{i \ell}$ é o efeito da interação do tratamento primário i com o tratamento terciário $\ell$;

$(\mathrm{bc})_{k \ell}$ é o efeito da interação do tratamento secundārio $\mathrm{k}$ com o tratamento terciário $\ell$;

$(a b c)_{i k l}$ é o efeito da interação tripla entre os tratamentos primário $i$, secundário $k$ e terciário $\ell$, e :

$e_{i j k \ell}$ é o erro residual a nível de. sub-subparcelas, também aleatório.

Em todas as deduções será utilizado o modelo fixo, o qual considera todos os parâmetros como fixos e apenas os erros $(a r)_{i j},(a b r)_{i k j} e e_{i j k \ell}$ como aleatórios, admitidos com média zero e variâncias $\sigma_{a r}^{2}, \sigma_{a b r}^{2}$ e $\sigma^{2}$ res pectivamente. 
O esquema de análise de variância, conside rando a perda de uma sub-subparcela é apresentado no Quadro I, a seguir:

QUADRO I - Esquema de Análise de Variância do Delineamento em Parcelas Sub-subdivididas com perda de uma unidade.

\begin{tabular}{|c|c|}
\hline Causas de Variação & Graus de Liberdade \\
\hline Blocos & $J-1$ \\
\hline Tratamentos (A) & $I-1$ \\
\hline Resíduo (a) & $(I-1)(J-1)$ \\
\hline 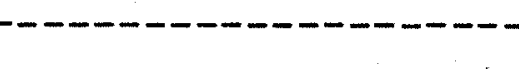 & - \\
\hline Parcelas & $(I J-1)$ \\
\hline Tratamentos (B) & $\mathrm{k}-\mathrm{l}$ \\
\hline Interação $A x B$ & $(I-1)(K-1)$ \\
\hline Resíduo (b) & $I(J-1)(K-1)$ \\
\hline Subparcelas & $(I J K-1)$ \\
\hline Tratamentos (C) & $L-1$ \\
\hline Interação $\mathrm{AxC}$ & $(I-1)(L-1)$ \\
\hline Interação $\mathrm{BxC}$ & $(K-1)(L-1)$ \\
\hline Interação $\mathrm{AxBxC}$ & $(I-1)(K-1)(L-1)$ \\
\hline Resíduo (c) & $I K(J-1)(L-1)-1$ \\
\hline
\end{tabular}


3.2:2. Decomposição da Soma de quadrado do Residuo (c)

A Soma de Quadrado do Resíduo (c) pode ser decomposta em componentes independentes associados a cada nível de combinação de tratamentos $A_{i} B_{k}$. Nessas condições a recuperação da informação da sub-subparcela $A_{i} B_{k} C_{\ell}$ do bloco $R_{j}$, torna-se viável pela minimização apenas do compo nente do resíduo (c) relativo ao núcleo $A_{i} B_{k}$ que contém a unidade perdida. Isto possibilita a utilização do estimador de uma parcela perdida dos experimentos em blocos casua lizados, associando cada combinação $A_{i} B_{k}$ à estrutura de um delineamento em blocos.

3.2.3. Variâncias de Contrastes entre Médias, Envol vendo uma Unidade Perdida

Tendo por base o modelo matemático e o estí mador da unidade perdida, serão estruturadas as variâncias de contrastes entre médias de tratamentos, envolvendo a sub-subparcela perdida.

Nas deduções apresentadas, não serão levados em conta a média geral e os duplos produtos, uma vez que na elaboração dos contrastes estes componentes se cance lam ou são nulos. 


\section{Assim considerar-se-á:}

$$
\hat{Y}=\hat{m}-\hat{m}_{1}
$$

onde $\hat{m}_{1}$ é a estimativa da média do tratamento que envolve a sub-subparcela perdida e, ainda:

$$
V(\hat{Y})=E[Q-E(\hat{Y})]^{2}
$$




\section{RESULTADOS E DISCUSSÃO}

4.1. Decomposição da Soma de Quadrado do Resíduo (c).

Partindo do modelo matemático, representatí vo de um delineamento em parcelas sub-subdivididas, a nível de cada combinação do tratamento primário $A_{i}$ com o tratamento $B_{k}$ tem-se:

\begin{tabular}{|c|c|c|c|c|c|}
\hline \multirow{2}{*}{$\begin{array}{c}\text { INIERAÇÃO } \\
\text { TRATAMENIOS } \\
\text { AxB } \\
\end{array}$} & \multirow{2}{*}{$\begin{array}{l}\text { TRATAMENTOS } \\
\text { TERCIARIOS }\end{array}$} & \multicolumn{4}{|c|}{ BLOCOS/REPEIIIÇ̃̃ES } \\
\hline & & $\mathrm{R}_{1}$ & $\mathrm{R}_{2}$ & $\cdots$ & $\mathrm{R}_{\mathrm{J}}$ \\
\hline \multirow{4}{*}{$A_{i} B_{k}$} & $c_{1}$ & $x_{i l k l}$ & $x_{i 2 k 1}$ & $\cdots$ & $x_{\text {iJkl }}$ \\
\hline & $\mathrm{C}_{2}$ & $x_{i \downarrow k 2}$ & $x_{i 2 k 2}$ & & $x_{i J k 2}$ \\
\hline & ... & ... & ... & ... & ... \\
\hline & $c_{L}$ & $x_{i l k L}$ & $x_{i 2 k L}$ & .. & $x_{i J k L}$ \\
\hline
\end{tabular}


Esta estrutura pode ser associada a de um experimento em blocos casualizados com L tratamentos e J blocos. Da análise de variância virá:

$$
\text { S.Q.Resíduo }{ }_{i k}=[\text { S.Q.Total }- \text { S.Q.Blocos - S.Q.Tratamentos (C) }]_{i k}
$$

mas :

$$
\text { S.Q.Total }=\sum_{j, \ell}^{\Sigma} x_{i j k \ell}^{2}-C_{1}
$$

onde:

$$
\begin{aligned}
& C_{1}=\frac{1}{J L} x_{i \cdot k .}^{2} \\
& \text { S.Q.Blocos }=\frac{1}{J} \sum_{l} x_{i \cdot k \ell}^{2}-C_{i} \\
& \text { S.Q.Tratamentos }(C)=\frac{1}{L} \sum_{j} x_{i j k}^{2} .-C_{1}
\end{aligned}
$$

Portanto:

S. Q. Resíduo ${ }_{i k}=\sum_{j, l} x_{i j k \ell}^{2}-\frac{1}{J} \sum_{\ell} x_{i . k \ell}^{2}-\frac{1}{L} \sum_{j} x_{i j k}^{2} .+\frac{1}{J L} x_{i . k}^{2}$.

Por outro lado, considerando o modelo completo de parcelas sub-subdivididas, a Soma de Quadrado do Resíduo (c) fica: 
S.Q.Resíduo (c) = S.Q.Total - S.Q.Subparcelas -

- S.Q.Tratamentos (C) - S.Q.Interação AxC -

- S.Q.Interação $\mathrm{BxC}$ - S.Q. Interação $\mathrm{AxBxC}$

mas :

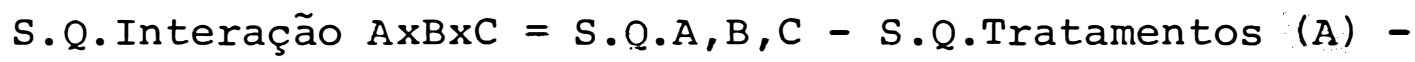

- S.Q.Tratamentos (B) - S.Q.Tratamentos (C) -

- S.Q.Interação AxB - S.Q.Interação AxC -

- S.Q.Interação $\mathrm{BxC}$

Substituindo em (2), e, sabendo que:

S.Q.A,B = S.Q.Tratamentos (A) + S.Q.Tratamentos (B) + + S.Q.Interação AxB,

virá:

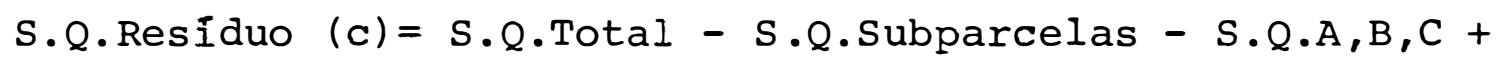
+ S.Q.A,B , 
32.

onde:

$$
\begin{aligned}
\text { S.Q.Total } & =\sum_{i j k \ell} x_{i j k \ell}^{2}-C \\
\text { S.Q.Subparcelas } & =\frac{1}{L} \sum_{i j k} x_{i j k .}^{2}-C \\
\text { S.Q.A,B,C } & =\frac{1}{J} \sum_{i k \ell} x_{i \cdot k \ell}^{2}-C \\
\text { S.Q.A,B } & =\frac{1}{J L} \sum_{i k} x_{i \cdot k}^{2} .-C
\end{aligned}
$$

Portanto:

S.Q.Residuo (c) $=\sum_{i j k \ell} x_{i j k \ell}^{2}-\frac{1}{L} \sum_{i j k} x_{i j k}^{2}-\frac{1}{J} \sum_{i k \ell} x_{i . k \ell}^{2}+\frac{1}{J L} \sum_{i k} x_{i . k .}^{2}$. Colocando $\underset{i k}{\sum}$ em evidência fica:

S.Q. Resíduo (c) $=\sum_{i k}\left(\sum_{j \ell} x_{i j k \ell}^{2}-\frac{1}{L} \sum_{j} x_{i j k}^{2} .-\frac{1}{J} \sum_{l} x_{i . k \ell}^{2}+\frac{l}{J L} x_{i . k .}^{2}\right)$

Identificando o conteúdo do parênteses com

(1), tem-se:

S.Q.Residuo (c) $=\sum_{i k}(\text { S.Q.Residuo })_{i k}=\sum_{i k}[S \cdot Q \cdot \text { Trat. (C) } x \text { Blocos }]_{i k}$ 
Em decorrência da independência dos componentes do Resíduo (c), associados aos núcleos $A_{i} B_{k}$, a recú peração da informação de uma sub-subparcela perdida é feita levando-se em conta exclusivamente o núcleo $A_{i} B_{k}$ afetado e utilizando o estimador de uma parcela perdida do delineamen to em blocos casualizados. Este procedimento viabiliza a es timação de diversas unidades perdidas em núcleos $A_{i} B_{k}$ distintos, considerando cada caso independentemente.

4.2. Esperança Matemática das Somas de Quadrados e Quadrados Médios.

A título de melhor visualização do desenvol vimento teórico, nas determinações das esperanças das somas de quadrados considerar-se-á a perda da sub-subparcela refe rente à combinação dos tratamentos $A_{1} B_{1} C_{1}$ do bloco $R_{1}$.

Assim, segundo PINHO (1973), a estimativa do valor de apenas uma sub-subparcela perdida pode ser calculado através da seguinte expressão geral:

$$
y=\frac{J\left(A_{i} B_{k} R_{j}\right)+L\left(A_{i} B_{k} C_{l}\right)-A_{i} B_{k}}{(J-1)(L-1)}
$$

onde:

y é o valor a ser estimado; .

J é o número de blocos; 
L é o número de tratamentos terciários (c);

$A_{j} B_{k} R_{j}$ é o total de todas as sub-subparcelas presentes na subparcela onde se perdeu a unidade;

$A_{i} B_{k} C_{l}$ é o total de todas as sub-subparcelas corresponden tes à combinação dos tratamentos $A_{i} E_{k} C_{l}$ relativos à sub--subparcela perdida.

$A_{i} B_{k}$ é o total de todas as sub-subparcelas presentes na combinação dos tratamentos $A_{i} B_{k}$ onde ocorreu a per da da unidade.

Ilustrando o caso suposto, na combinação $\mathrm{A}_{1} \mathrm{~B}_{1}$, associada a (1), tem-se:

\begin{tabular}{|c|c|c|c|c|}
\hline \multirow{2}{*}{$\begin{array}{l}\text { TRATAMENTOS } \\
\text { TERCIARIOS }\end{array}$} & \multicolumn{4}{|c|}{ BLOCOS } \\
\hline & $\mathrm{R}_{1}$ & $\mathrm{R}_{2}$ & $\cdots$ & $\mathrm{R}_{\mathrm{J}}$ \\
\hline$C_{1}$ & $Y$ & & ${ }_{1} \mathrm{~B}_{1} \mathrm{C}_{1}$ & . \\
\hline $\mathrm{C}_{2}$ & & & & \\
\hline $\begin{array}{c}\cdots \\
C_{L}\end{array}$ & $A_{1} B_{1} R_{1}$ & & $\mathrm{R}$ & \\
\hline
\end{tabular}


de tal forma que:

$$
A_{1} B_{1}=A_{1} B_{1} R_{1}+A_{1} B_{1} C_{1}+R
$$

onde:

$R$ é o total das observações presentes na combinação $A_{1} B_{1}$ exceto as relativas à $A_{1} B_{1} C_{1}$ e à $A_{1} B_{1} R_{1}$. Substituindo.(2) em (1), para este caso em apreço, tem-se:

$$
Y=\frac{(J-1) A_{1} B_{1} R_{1}+(L-1) A_{1} B_{1} C_{1}-R}{(J-1)(L-1)}
$$

Expressando os valores de $A_{1} B_{1} R_{1}, A_{1} B_{1} C_{1} e$ R em função do modelo matemático virá:

$$
\begin{aligned}
A_{1} B_{1} R_{1}=\sum_{\ell=2}^{L} x_{111 \ell}= & (L-1) a_{1}+(L-1) r_{1}+(L-1)(a r)_{11}+(L-1) b_{1}+ \\
& 1 \\
& +(L-1)(a b)_{11}+(L-1)(a b r)_{111}+\sum_{\ell=1}^{L} c_{\ell}+ \\
& +\sum_{\ell=1}^{L}(a c)_{1 \ell}+\sum_{\ell=1}^{L}(b c)_{1 \ell}+\sum_{\ell=1}^{L}(a b c)_{11 \ell}+ \\
& +\sum_{\ell=2}^{L} e_{111 \ell}-c_{1}-(a c)_{11}-(b c)_{11}- \\
& -(a b c)_{111}
\end{aligned}
$$




$$
\begin{aligned}
& A_{1} B_{1} C_{1}=\sum_{j=2}^{J} x_{1 j 11}=(J-1) a_{1}+\sum_{j=1}^{J} r_{j}+\sum_{j=2}^{J}(a r)_{1 j}+(J-1) b_{1}+ \\
& +(J-1)(a b)_{11}+\sum_{j=2}^{J}(a b r)_{11 j}+(J-1) c_{1}+ \\
& +(J-1)_{11}+(J-1)_{11}+ \\
& +(J-1)(a b c)_{111}+\sum_{j=2}^{J} e_{1 j 11}-r_{1} \\
& R=\sum_{j=2}^{J} \sum_{\ell=2}^{L} x_{1 j_{1 \ell}}=(J-1)(L-1) a_{1}+(L-1) \sum_{j=1}^{J} r_{j}+ \\
& +(L-1)_{j=2}^{J}(\operatorname{ar})_{1 j}+(J-1)(L-1) b_{1}+ \\
& +(J-1)(L-1)(a b)_{11}+(L-1)_{j=2}^{J}(a b r)_{11 j}+ \\
& +(J-1) \sum_{\ell=1}^{L} c_{\ell}+(J-1) \sum_{\ell=1}^{L}(a c)_{1 \ell}+ \\
& +(J-1) \sum_{\ell=1}^{L}(b c)_{1 \ell}+(J-1)_{\ell=1}^{L}(a b c)_{11 \ell}+ \\
& +\sum_{j=2}^{J} \sum_{\ell=2}^{L} e_{1 j 1 \ell}-(L-1) r_{1}-(J-1) c_{1}- \\
& -(J-1)_{(a c)_{11}}-(J-1)(b c)_{11}-(J-1)(a b c)_{111}
\end{aligned}
$$


Substituindo essas expressóes em (3), aplicando as seguintes restrições:

$$
\begin{aligned}
& \sum_{i}^{\sum a_{i}}=0 ; \sum_{j}^{\sum r_{j}}=0 ; \sum_{k}^{\sum b_{k}}=0 ; \sum_{i}^{\sum(a b)_{i k}}=\sum_{k}^{\sum(a b)_{i k}}=\sum_{i k}^{\sum(a b)_{i k}}=0 ; \sum_{l}^{\sum c_{l}}=0
\end{aligned}
$$

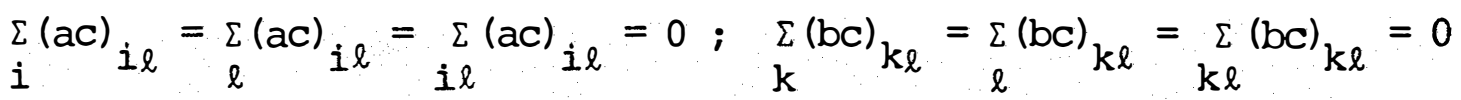

$$
\begin{aligned}
& \sum_{i}(a b c){ }_{i k \ell}=\sum_{k}(a b c)_{i k \ell}=\sum_{\ell}(a b c)_{i k \ell}=\sum_{i k}(a b c)_{i k \ell}=\sum_{i \ell}(a b c){ }_{i k \ell}=\sum_{k \ell}(a b c){ }_{i k \ell}= \\
& =\sum_{i k \ell}(a b c)_{i k \ell}=0,
\end{aligned}
$$

e simplificando fica:

$$
\begin{aligned}
& y=a_{1}+r_{1}+(a r)_{11}+b_{1}+(a b)_{11}+(a b r)_{111}+c_{1}+(a c)_{11}+(b c)_{11}+ \\
& +(a b c)_{111}+\frac{\sum_{j=2}^{J} e_{1 j 11}}{J-1}+\frac{\sum_{\ell=2}^{L} e_{111 \ell}}{L-1}-\frac{\sum_{j=2}^{J} \sum_{\ell=2}^{L} e_{1 j 1 \ell}}{(J-1)(L-1)}
\end{aligned}
$$

onde:

$$
\begin{aligned}
& \sum_{j=2}^{J} e_{1 j l 1} \text { é a soma dos }(J-1) \text { erros associados às sub- } \\
& \text { - subparcelas presentes na combinação de trä } \\
& \text { tamentos } \mathrm{A}_{1} \mathrm{~B}_{1} \mathrm{C}_{1} \text {; . } \\
& \sum_{\ell=2}^{L} e_{111 \ell} \text { é a soma dos (L-1) erros associados às sub- } \\
& \text { - subparcelas presentes na subparcela } A_{1} B_{1} R_{1} \text {, } \\
& \text { e : }
\end{aligned}
$$


J L

$\Sigma \quad \sum e_{1 j l \ell}$ é a soma dos $(J-1)(L-1)$ erros associados às $j=2 \ell=21 j 1 \ell$ subparcelas presentes no núcleo $A_{1} B_{1}$ mas que não receberam o tratamento $C_{1}$ e não estão no bloco $R_{-1}$.

As exoressões correspondentes às esperanças matemáticas das Somas de Quadrados e Quadrados Médios das causas de variação foram as que se seguem.

$$
\begin{aligned}
& \text { 4.2.1. Total } \\
& E(\text { S.Q.Total })=E\left(\sum_{i j k \ell}^{\sum^{\prime}} x_{i j k \ell}^{2}+y^{2}\right)-E(C)
\end{aligned}
$$

onde:
$\sum_{i j k \ell}^{\prime} x_{i j k \ell}^{2}$ representa a soma de quadrado de todas as sub-subparcelas do ensaio, exceto daquela referente à perda.

$$
\text { Assim tem-se: }
$$

i) $E(C)=\frac{E\left(\sum_{i j k \ell}^{\prime} x_{i j k \ell}+y\right)^{2}}{}$ 
onde:

$$
\begin{aligned}
& \underset{i j k \ell}{\sum^{\prime}} x_{i j k \ell}+y=J K L \sum_{i} a_{i}+I K L \sum_{j} r_{j}+K L \sum_{i j}(a r)_{i j}+I J L \sum_{k} b_{k}+ \\
& +\pi \sum_{i k}(a b)_{i k}+I \sum_{i j k}(a b r)_{i k j}+\operatorname{IJK} \sum_{\ell} c_{\ell}+ \\
& +J K \sum_{i \ell}(a c)_{i l}+I J \sum_{k \ell}(b c)_{k \ell}+J \sum_{i k \ell}(a b c)_{i k \ell}+ \\
& +\sum_{i j k \ell}^{\prime} e_{i j k l}+\frac{\sum_{\ell=2}^{L} e_{111 \ell}}{L-1}+\frac{\sum_{j=2}^{J} e_{1 j 11}}{J-1}- \\
& \sum_{\substack{J=2 \\
l=2}}^{L} e_{1 j l \ell} \\
& (J-1)(L-1)
\end{aligned}
$$

onde:

$$
\begin{aligned}
\sum_{i j k \ell}^{\prime} e_{i j k l} & \text { representa a soma dos (IJKL - I) erros as- } \\
& \text { sociados às sub-subparcelas presentes. Mas } \\
& \text { esses erros podem ser decompostos da se- } \\
& \text { guinte maneira: }
\end{aligned}
$$

$$
\begin{aligned}
\sum_{i j k \ell}^{\prime} e_{i j k l}= & \sum_{i j k \ell}^{\prime \prime} e_{i j k \ell}+\sum_{j=2}^{J} \sum_{\ell=2}^{L} e_{1 j l \ell}+\sum_{j=2}^{J} e_{1 j l l}+ \\
& +\sum_{\ell=2}^{L} e_{111 \ell}
\end{aligned}
$$


e ainda:

$$
\begin{array}{cl}
\sum_{i j k \ell}^{\prime \prime} e_{i j k \ell} & \text { é a soma dos } J L(I K-1) \text { erros associados } \\
& \text { aos demais núcleos } A_{i} B_{k} \text { onde não houve per } \\
& \text { da de sub-subparcelas. }
\end{array}
$$

Substituindo esses erros na equação (5), a plicando a esperança e impondo as restrições tem-se:

$$
\begin{aligned}
E\left(\sum_{i j k \ell}^{\prime} x_{i j k \ell}+y\right)^{2}= & I J K^{2} L^{2} \sigma_{a r}^{2}+I J K L^{2} \sigma_{a b r}^{2}+\frac{L^{2}}{L-1} \sigma^{2}+\frac{J^{2}}{J-1} \sigma^{2}+ \\
& +\frac{[(J-I)(L-I)-I]^{2}}{(J-I)(L-I)} \sigma^{2}+J L(I K-I) \sigma^{2}
\end{aligned}
$$

pois:

$$
\begin{aligned}
& \left.E\left(a_{i}^{2}\right)=a_{i}^{2} ; E\left(r_{j}^{2}\right)=r_{j}^{2} ; E(b)_{k}^{2}\right)=b_{k}^{2} ; E(a b)_{i k}^{2}=(a b)_{i k}^{2} ; \\
& E\left(c_{l}^{2}\right)=c_{l}^{2} ; E(a c)_{i l}^{2}=(a c)_{i l}^{2} ; E(b c)_{k l}^{2}=(b c)_{k l}^{2} ; \\
& E(a b c)_{i k l}^{2}=(a b c)_{i k \ell}^{2} ; E(a r)_{i j}^{2}=\sigma_{a r}^{2} ; E(a b r)_{i k j}^{2}=\sigma_{a b r}^{2} ; \\
& E(e)_{i j k l}^{2}=\sigma^{2}
\end{aligned}
$$


Assim:

$\frac{E\left(\sum_{i j k \ell}^{\prime} x_{i j k \ell}+y\right)^{2}}{I J K L}=K L \sigma_{a r}^{2}+L \sigma_{a b r}^{2}+\frac{J L+I J K L(J-I)(L-I)}{\operatorname{IJKL}(J-I)(L-1)} \sigma^{2}$

ou ainda:

$$
\begin{aligned}
& E(C)=K L \sigma_{a r}^{2}+L \sigma_{a b r}^{2}+\left[1+\frac{1}{\operatorname{IK}(J-1)(L-1)}\right] \sigma^{2} \quad(\sigma) \\
& \text { ii) } E\left(\sum_{i j k \ell}^{\sum^{\prime}}\left(x_{i j k \ell}^{2}\right)+E\left(y^{2}\right)=\sum_{i j k \ell}^{\sum^{\prime}}\left[E\left(x_{i j k \ell}\right)^{2}\right]+E\left(y^{2}\right)\right.
\end{aligned}
$$

Como:

$$
\begin{aligned}
E\left(y^{2}\right)= & a_{1}^{2}+r_{1}^{2}+\sigma_{a r}^{2}+b_{1}^{2}+(a b)_{11}^{2}+\sigma_{a b r}^{2}+c_{1}^{2}+(a c)_{11}^{2}+ \\
& +(b c)_{11}^{2}+(a b c)_{111}^{2}+\frac{J+L-1}{(J-1)(L-1)} \sigma^{2}
\end{aligned}
$$

e:

$$
\begin{aligned}
E\left(x_{i j k \ell}\right)^{2}= & E\left[a_{i}+r_{j}+(a r)_{i j}+b_{k}+(a b)_{i k}+(a b r)_{i k j}+c_{\ell}+\right. \\
& \left.+(a c)_{i \ell}+(b c)_{k \ell}+(a b c)_{i k \ell}+e_{i j k \ell}\right]^{2} .
\end{aligned}
$$

Desenvolvendo o quadrado, aplicando o somatório e acrescentando $E\left(y^{2}\right)$ fica: 
$\underset{i j k \ell}{\sum^{\prime}}\left[E\left(x_{i j k \ell}\right)^{2}\right]+E\left(y^{2}\right)=J K L \underset{i}{\sum_{i}} a_{i}^{2}+I K L \underset{j}{\sum_{j}} r_{j}^{2}+I J K L \sigma_{a r}^{2}+I J L \underset{k}{\sum} b_{k}^{2}+$

$$
\begin{aligned}
& +J L \sum_{i k}(a b)_{i k}^{2}+I J K L \sigma_{a b r}^{2}+I J K \sum_{l} c_{l}^{2}+ \\
& +J K \sum_{i l}(a c)_{i l}^{2}+I J \sum_{k \ell}(b c)_{k \ell}^{2}+J \sum_{i k l}(a b c)_{i k l}^{2}+ \\
& +\left[(I J K L-1)+\frac{J+L-1}{(J-1)(I-1)}\right] \sigma^{2}
\end{aligned}
$$

$$
\text { Substituindo (6) e (7) em (I) tem-se: }
$$

$$
\begin{aligned}
E \text { (S.Q.TotaI) }= & J K \sum_{i} a_{i}^{2}+I K L \sum_{j} r_{j}^{2}+K L(I J-1) \sigma_{a r}^{2}+I J \sum_{k} b_{k}^{2}+ \\
& +J L \sum_{i k}(a b)_{i k}^{2}+I(I J K-1) \sigma_{a b r}^{2}+I J K \sum_{\ell} c_{l}^{2}+J K \sum_{i l}(a c)_{i l}^{2}+ \\
& +I J \sum_{k l}(b c)_{k \ell}^{2}+J \sum_{i k \ell}(a b c)_{i k \ell}^{2}+ \\
& +\left[(I J K L-3)+\frac{(I J K L-1)}{I K(J-1)(I-1)}\right] \sigma^{2}
\end{aligned}
$$


43.

4.2.2. Blocos

$E(S \cdot Q \cdot B l o c o s)=\frac{1}{I K L} E\left(\sum_{j=2}^{J} R_{j}^{2}\right)+\frac{1}{I K L} E\left(R_{1}+y\right)^{2}-E(C)$

onde:

$$
\begin{aligned}
& R_{j} \text { é o toṫal de todas as sub-subparcelas do bloco } j \text {, } \\
& R_{1} \text { é o total de todas as sub-subparcelas presentes no } \\
& \text { bloco } 1 \text { onde ocorreu a perda. } \\
& \text { Assim, } \\
& R_{j}=\sum_{i k \ell} x_{i j k l}=K L \sum_{i} a_{i}+I K L r_{j}+K L \sum_{i}(a r)_{i j}+I L \sum_{k}^{\sum} b_{k}+L \sum_{i k}(a b)_{i k}+ \\
& +\mathrm{L} \sum_{i k}(a b r)_{i k j}+I K \sum_{\ell} c_{\ell}+K \sum_{i \ell}(a c)_{i \ell}+I \sum_{k \ell}(b c)_{k \ell}+ \\
& +\sum_{i k \ell}(a b c)_{i k \ell}+\sum_{i k \ell} e_{i j k \ell} . \\
& E\left(R_{j}^{2}\right)=I^{2} K^{2} L^{2} r_{j}^{2}+I K^{2} L^{2} \sigma_{a r}^{2}+I K L^{2} \sigma_{a b r}^{2}+I K L \sigma^{2}
\end{aligned}
$$


Desenvolvendo o somatório em relação à $j$,

virá:

$$
\begin{aligned}
\frac{1}{I K L} \sum_{j=2}^{J}\left[E\left(R_{j}^{2}\right)\right]= & I K L \sum_{j=2}^{J} r_{j}^{2}+K L(J-1) \sigma_{a r}^{2}+ \\
& +L(J-1) \sigma_{a b r}^{2}+(J-1) \sigma^{2} .
\end{aligned}
$$

Para o bloco onde houve a perda de informação tem-se:

$$
\begin{aligned}
& R_{1}+y=K L \underset{i}{\sum_{i}} a_{i}+I K L r_{1}+K L \underset{i}{\sum}(a r)_{i l}+I L \underset{k}{\sum} b_{k}+L \sum_{i k}(a b)_{i k}+ \\
& +\mathrm{L} \sum_{i k}(a b r)_{i k 1}+I K \sum_{\ell} c_{\ell}+K \sum_{i \ell}(a c)_{i \ell}+I \sum_{k \ell}(b c)_{k \ell}+ \\
& +\sum_{i k \ell}(a b c){ }_{i k \ell}+\sum_{i k \ell} e_{i 1 k \ell}+\frac{\sum_{\ell=2}^{L} e_{111 \ell}}{L-1}+\frac{\sum_{j=2}^{J} e_{1 j 11}}{J-1}- \\
& -\frac{\sum_{j=2}^{J} \sum_{=2}^{L} e_{1 j 1 \ell}}{(J-1)(L-1)}
\end{aligned}
$$

onde:

$$
\begin{aligned}
\sum_{i k \ell} e_{i l k \ell} \text { é a soma dos (IKL-I) erros associados às } \\
\text { sub-subparcelas presentes no bloco onde 으 } \\
\text { correu a perda da unidade, e que pode ser } \\
\text { decomposto em: }
\end{aligned}
$$




$$
\sum_{i k \ell} e_{i i k \ell}=\sum_{i k \ell}^{\prime} e_{i l k \ell}+\sum_{k \ell} e_{i l k \ell}+\sum_{\ell=2}^{L} e_{111 \ell}
$$

onde:

$$
\begin{aligned}
& \Sigma^{\prime} e_{i l k \ell} \text { é a soma dos } \mathrm{KL}(I-1) \text { erros associados às } \\
& \sum_{k \ell} e_{i l k l}
\end{aligned}
$$

Substituindo esses erros, elevando ao quadrado, aplicando a esperança e considerando as restrições, fica:

$$
\begin{aligned}
E\left(R_{1}+y\right)^{2}= & I^{2} K^{2} L^{2} r_{1}^{2}+I K^{2} L^{2} \sigma_{a r}^{2}+I K L^{2} \sigma_{a b r}^{2}+\frac{L^{2}}{I-1} \sigma^{2}+\frac{\sigma^{2}}{J-1}+ \\
& +L(K-I) \sigma^{2}+K L(I-I) \sigma^{2}+\frac{\sigma^{2}}{(J-I)(L-I)} .
\end{aligned}
$$

Simplificando virá:

$$
\frac{E\left(R_{1}+y\right)^{2}}{I K L}=I K L r_{1}^{2}+K L \sigma_{a r}^{2}+I \sigma_{a b r}^{2}+\left[1+\frac{J}{I K(J-I)(L-I)}\right] \sigma^{2}
$$


Substituindo (6), (8) e (9) em (II) tem-se:

$E(S \cdot Q \cdot B l o c o s)=I K L \sum_{j} r_{j}^{2}+J K L \sigma_{a r}^{2}+J L \sigma_{a b r}^{2}+$

$$
\begin{aligned}
& +\left[J+\frac{J}{I K(J-I)(L-1)}\right] \sigma^{2}-K L \sigma_{a r}^{2}-L \sigma_{a b r}^{2} \\
& -\left[1+\frac{1}{I K(J-1)(L-1)}\right] \sigma^{2} .
\end{aligned}
$$

Portanto:

$$
\begin{aligned}
E(S \cdot Q \cdot B l o c o s)= & I K L \sum_{j} r_{j}^{2}+K L(J-1) \sigma_{a r}^{2}+L(J-1) \sigma_{a b r}^{2}+ \\
& +\left[(J-1)+\frac{(J-1)}{I K(J-1)(L-1)}\right] \sigma^{2} \cdot
\end{aligned}
$$

Como ao componente "blocos" estão associados J-1 graus de liberdade, tem-se:

$$
E(Q . M . B l o c o s)=\frac{E(S \cdot Q \cdot B l o c o s)}{J-1},
$$

ou ainda:

$$
E(Q . M . B l o 00 s)=\frac{I K L}{J-1} \sum_{j} r_{j}^{2}+K L \sigma_{a r}^{2}+L \sigma_{a b r}^{2}+\left[1+\frac{1}{I K(J-1)(I-l)}\right] \sigma^{2}
$$


$E[$ S.Q.Tratamentos $(A)]=\frac{I}{J K L} E\left(\sum_{i=2}^{I} A_{i}^{2}\right)+\frac{I}{J K L} E\left(A_{1}+y\right)^{2}-E(C)$

onde:

$A_{i}$ é $~$ total de todas as sub-subparcelas que compõem o tratamento $A_{i}$, exceto aquele onde houve a per da da informação, e:

$A_{1}$ é o total de todas as sub-subparcelas do tratamen to $\mathrm{A}_{1}$ onde ocorreu a perda.

\section{Como,}

$A_{i}=\sum_{j k \ell} x_{i j k \ell}=J K L a_{i}+K L \sum_{j}^{\sum} r_{j}+K L \underset{j}{\sum}(a r)_{i j}+J L \underset{k}{\sum} b_{k}+$ $+J L \underset{k}{\sum}(a b)_{i k}+L \underset{j k}{\Sigma}(a b r)_{i k j}+J K \sum_{\ell} c_{\ell}+$ $+J K_{\ell}^{\sum(a c)_{i l}}+J \underset{k \ell}{\sum_{k l}(b c)_{k \ell}}+J \underset{k \ell}{\sum}(a b c)_{i k \ell}+$ $+\sum_{j k \ell} e_{i j k \ell}$

elevando ao quadrado, aplicando a esperança e impondo as res trições, fica:

$$
E\left(A_{i}^{2}\right)=J^{2} K^{2} L^{2} a_{i}^{2}+J K^{2} L^{2} \sigma_{a r}^{2}+J K L^{2} \sigma_{a b r}^{2}+J K L \sigma^{2}
$$


Desenvolvendo virá:

$$
\begin{aligned}
\frac{1}{J K L} \sum_{i=2}^{I}\left[E\left(A_{i}^{2}\right)\right]= & J K L \sum_{i=2}^{I} a_{i}^{2}+K L(I-1) \sigma_{a r}^{2}+ \\
& +L(I-1) \sigma_{a b r}^{2}+(I-1) \sigma^{2}
\end{aligned}
$$

Para o tratamento onde ocorreu a perda da sub-subparcela tem-se:

$$
\begin{aligned}
& A_{1}+y=J K L a_{l}+K L \sum_{j} r_{j}+K L \sum_{j}(a r)_{1 j}+J L \sum_{k} b_{k}+ \\
& +J I_{k}(a b)_{l k}+L \sum_{j k}(a b r)_{1 k j}+J K \sum_{\ell} c_{\ell}+
\end{aligned}
$$

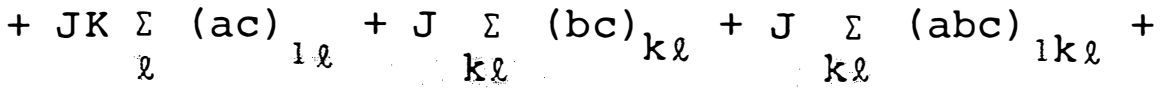

$$
\begin{aligned}
& +\sum_{j k \ell} e_{1 j k l}+\frac{\sum_{j=2}^{J} e_{1 j 11}}{J-1}+\frac{\sum_{\ell=2}^{L} e_{111 \ell}}{L-1}- \\
& -\frac{\sum_{j=2}^{J} \sum_{\ell=2}^{L} e_{1 j l \ell}}{(J-1)(L-1)}
\end{aligned}
$$

onde:

$$
\begin{aligned}
\sum_{j k \ell} e_{l j k \ell} & \text { é a soma dos }(J K L-1) \text { erros associados às } \\
& \text { sub-subparcelas presentes no tratamento } A_{1}, \\
& \text { onde ocorreu a perda da observação. Esse } \\
& \text { componente pode ser desdobrado em: }
\end{aligned}
$$




$$
\underset{j k \ell}{\sum} e_{1 j k \ell}=\underset{j k \ell}{\Sigma^{\prime}} e_{1 j k \ell}+\underset{j \ell}{\sum} e_{1 j k \ell}+\sum_{\ell=2}^{L} e_{111 \ell}
$$

onde:

$$
\begin{aligned}
\sum^{\prime} e_{1 j k l} & \text { é a soma dos } \mathrm{JL}(\mathrm{K}-1) \text { erros associados às } \\
& \text { sub-subparcelas que estão no tratamento } \mathrm{A}_{1} \\
& \text { mas não receberam o tratamento } B_{1} \text { corres- } \\
& \text { pondente à perda. } \\
\sum_{j \ell} e_{1 j k \ell} & \text { é a soma dos } L(J-1) \text { erros associados às } \\
& \text { sub-subparcelas que estão nas demais sub- } \\
& \text { parcelas, que não perderam a observação, do } \\
& \text { núcleo } A_{1} B_{1} .
\end{aligned}
$$

E assim,

$$
\begin{aligned}
E\left(A_{1}+y\right)^{2}= & J^{2} K^{2} L^{2} a_{1}^{2}+J K^{2} L^{2} \sigma_{a r}^{2}+J K L^{2} \sigma_{a b r}^{2}+\frac{L^{2}}{L-1} \sigma^{2}+ \\
& +\frac{\sigma^{2}}{J-1}+J L(K-I) \sigma^{2}+L(J-I) \sigma^{2}+\frac{\sigma^{2}}{(J-1)(L-1)}
\end{aligned}
$$

ou ainda:

$\frac{E\left(A_{1}+y\right)^{2}}{J K L}=J K L a_{1}^{2}+K L \sigma_{a r}^{2}+L \sigma_{a b r}^{2}+\left[1+\frac{I}{I K(J-I)(L-1)}\right] \sigma^{2}$ 
Pela substituição de (6), (10) e (11) em (III) virá:

$$
\begin{aligned}
& E[\text { S.Q.Tratamentos }(A)]=J K L \underset{i}{\sum_{i}} a_{i}^{2}+K L(I-1) \sigma_{a r}^{2}+L(I-1) \underset{a b r}{\sigma^{2}}+ \\
& +\left[(I-I)+\frac{(I-1)}{I K(J-1)(L-1)}\right] \sigma^{2}
\end{aligned}
$$

e,

$$
\text { E[Q.M.Tratamentos }(A)]=\frac{E[\text { S.Q.Tratamento }(A)]}{I-1} \text {. }
$$

ou seja:

$$
\begin{aligned}
& E[Q . M . T r a t a m e n t o s(A)]=\frac{J K L}{I-1} \sum_{i} a_{i}^{2}+K L \sigma_{a r}^{2}+L \sigma_{a b r}^{2}+ \\
& +\left[1+\frac{I}{\operatorname{IK}(J-1)(L-1)}\right] \sigma^{2} .
\end{aligned}
$$

4.2.4. Parcetas

$E(S . Q . P a r c e l a s)=\frac{1}{K L} E\left[\sum_{i j}\left(A_{i} R_{j}\right)^{2}\right]+\frac{1}{K L} E\left(A_{1} R_{I}+y\right)^{2}-E(C)$ 
onde :

$A_{i} R_{j} \quad$ é $\circ$ total de todas as sub-subparcelas que com põem as parcelas $A_{i} R_{j}$ não afetadas pela perda de observação

$A_{1} R_{1}$ é 0 total de todas as sub-subparcelas presentes na parcela onde houve a perda de informação.

A expressão do modelo que representa cada parcela onde não ocorreu perda é dada por:

$$
\begin{aligned}
& A_{i} R_{j}=\underset{k \ell}{\sum} x_{i j k l}=K L a_{i}+K L r_{j}+K L(a r)_{i j}+L \underset{k}{\sum} b_{k}+ \\
& +\underbrace{\sum_{i k}}_{k}(a b)_{i k}+\sum_{k}^{\sum_{1}}(a b r)_{i k j}+k \sum_{\ell} c_{\ell}+ \\
& +k \sum_{\ell}(a c)_{i l}+\sum_{k l}(b c)_{k l}+\sum_{k \ell}(a b c)_{i k \ell}+ \\
& +\sum_{k \ell} e_{i j k \ell} \cdot
\end{aligned}
$$

Elevando ao quadrado, aplicando a esperança e impondo as restrições, tem-se:

$E\left(A_{i} R_{j}\right)^{2}=K^{2} L^{2} a_{i}^{2}+K^{2} L^{2} r_{j}^{2}+K^{2} L^{2} \sigma_{a r}^{2}+K L^{2} \sigma_{a b r}^{2}+K L \sigma^{2} \cdot$ Desenvolvendo o somatório em relação a i e j, virá: 
52 .

a) para as parcelas independentes de $A_{1}$ :

$$
\begin{aligned}
\frac{1}{K L} \sum_{i=2}^{I} \sum_{j}\left[E\left(A_{i} R_{j}\right)^{2}\right]= & J K L \sum_{i=2}^{I} a_{i}^{2}+K L(I-1) \sum_{j} r_{j}^{2}+ \\
& +J K L(I-1) \sigma_{a r}^{2}+J L(I-1) \sigma_{a b r}^{2}+ \\
& +J(I-1) \sigma^{2}
\end{aligned}
$$

b) para as parcelas relativas à $A_{1}$, exceto aquela associada à perda da unidade:

$$
\begin{aligned}
\frac{l}{K L} \sum_{j=2}^{J}\left[E\left(A_{1} R_{j}\right)^{2}\right]= & K L(J-1) a_{1}^{2}+K L \sum_{j=2}^{J} r_{j}^{2}+K L(J-1) \sigma_{a r}^{2}+ \\
& +L(J-1) \sigma_{a b r}^{2}+(J-1) \sigma^{2}
\end{aligned}
$$

Para a parcela onde ocorreu a nerda da observação fica:

$$
\begin{aligned}
& A_{1} R_{1}+y=K L a_{1}+K L r_{1}+K L(a r)_{11}+L \sum_{k} b_{k}+L \sum_{k}^{\sum}(a b)_{1 k}+L \underset{k}{\sum}(a b r)_{1 k 1}+ \\
& +\mathrm{K} \sum_{\ell} c_{\ell}+\mathrm{K} \sum_{\ell}(\mathrm{ac})_{1 \ell}+\underset{\mathrm{k} \ell}{\sum}(\mathrm{bc})_{\mathrm{k} \ell}+\underset{\mathrm{k} \ell}{\sum}(\mathrm{abc})_{1 \mathrm{k}_{\ell}}+\underset{\mathrm{k} \ell}{\sum} \mathrm{e}_{11 \mathrm{k} \ell}+ \\
& +\frac{\sum_{j=2}^{J} e_{1 j 11}}{J-1}+\frac{\sum_{\ell=2}^{L} e_{111 \ell}}{L-1}-\frac{\sum_{j=2}^{J} \sum_{\ell=2}^{L} e_{1 j 1 \ell}}{(J-1)(L-1)}
\end{aligned}
$$


onde:

$$
\begin{aligned}
\sum_{k \ell}^{\sum} e_{11 k \ell} & \text { é a soma dos }(\mathrm{KL}-1) \text { erros associados às sub- } \\
& \text { - subparcelas presentes na parcela onde ocor } \\
& \text { reu a perda, e que pode ser decomposto em: } \\
\sum_{k \ell}^{\sum} e_{11 k \ell}= & \sum_{k \ell}^{\prime} e_{11 k \ell}+\sum_{\ell=2}^{L} e_{111 \ell} .
\end{aligned}
$$

Substituindo, elevando ao quadrado, aplican do a esperança e impondo as restrições anteriores, virá:

$$
\begin{aligned}
E\left(A_{1} R_{1}+y\right)^{2}= & K^{2} L^{2} a_{1}^{2}+K^{2} L^{2} r_{1}^{2}+R^{2} L^{2} \sigma_{a r}^{2}+K L^{2} \sigma_{a b r}^{2}+ \\
& +\frac{L^{2}}{L-1} \sigma^{2}+\frac{\sigma^{2}}{J-1}+L(K-I) \sigma^{2}+\frac{\sigma^{2}}{(J-I)(L-1)},
\end{aligned}
$$

donde :

$$
\begin{aligned}
\frac{E\left(A_{1} R_{1}+Y\right)^{2}}{K L}= & K L a_{1}^{2}+K L r_{1}^{2}+K L \sigma_{a r}^{2}+L \sigma_{a b r}^{2}+ \\
& +\left[1+\frac{I J}{I K(J-I)(L-I)}\right] \sigma^{2}
\end{aligned}
$$


54.

Substituindo (6), (a), (b) e (12) em (IV),

virá:

$E(S \cdot Q \cdot P a r c e l a s)=J K L \sum_{i} a_{i}^{2}+\operatorname{IKL} \sum_{j .} r_{j}^{2}+K L(I J-1) \sigma_{a r}^{2}+$

$$
+L(I J-1) \sigma_{a b r}^{2}+\left[(I J-1)+\frac{(I J-1)}{I K(J-1)(L-1)}\right] \sigma^{2}
$$

4.2.5. Residuo (a)

$\mathrm{E}[\mathrm{S} \cdot \mathrm{Q} \cdot \operatorname{Residuo~(a)]~=~E(S.Q.Parcelas)~-~E(S.Q.Blocos)~-~}$

$$
\text { - E[S.Q.Tratamentos (A)] }
$$

Substituindo as expressões resultantes de (IV), (II) e (III), respectivamente, em (V), virá:

$\mathrm{E}[\mathrm{S} \cdot \mathrm{Q} \cdot \operatorname{Residuo}(\mathrm{a})]=\mathrm{KL}(\mathrm{I}-1)(\mathrm{J}-1) \sigma_{\mathrm{ar}}^{2}+\mathrm{L}(\mathrm{I}-1)(\mathrm{J}-1) \sigma_{\mathrm{abr}}^{2}+$

$$
+\left[(I-1)(J-1)+\frac{(I-1)(J-1)}{I K(J-1)(L-1)}\right] \sigma^{2}
$$

e então:

$$
E\left[Q \cdot M \cdot \operatorname{Residuo~(a)]}=\frac{E[S \cdot Q \cdot \operatorname{Residuo~(a)]}}{(I-1)(J-1)}\right.
$$


ou seja:

$E[Q . M . R e s i d u o(a)]=K L \sigma_{a r}^{2}+L \sigma_{a b r}^{2}+\left[1+\frac{1}{I K(J-1)(L-1)}\right] \sigma^{2}$ Como se observa, esta expressão é idêntica àquela da esperança da correção (6) apresentada anteriormen te.

4.2.6. Tratamentos (B)

$E[S \cdot Q \cdot T r a t a m e n t o s(B)]=\frac{1}{I J L} E\left(\sum_{k=2}^{K} B_{k}^{2}\right)+\frac{1}{I J L} E\left(B_{1}+y\right)^{2}-$

onde:

$\mathrm{B}_{\mathrm{k}}$ é a soma de todas as sub-subparcelas que compõem o total de cada tratamento $\mathrm{B}_{\mathrm{k}}$, exceto aquele onde houve a perda da observação, e:

B é a soma de todas as sub-subparcelas presentes no tratamento $B_{1}$ que perdeu a sub-subparcela. 
Mas:

$$
\begin{aligned}
& B_{k}=\sum_{i j \ell} x_{i j k \ell}=J L \sum_{i} a_{i}+I L \sum_{j} r_{j}+L \sum_{i j}(a r)_{i j}+I J L b_{k}+ \\
& +J L \sum_{i}(a b)_{i k}+L \sum_{i j}(a b r)_{i k j}+I J \sum_{\ell} c_{\ell}+ \\
& +J \sum_{i \ell}(\mathrm{ac})_{i \ell}+I J \sum_{\ell}(\mathrm{bc})_{\mathrm{k} \ell}+ \\
& +J \sum_{i \ell}(a b c) i k \ell+\sum_{i j \ell} e_{i j k \ell} .
\end{aligned}
$$

Elevando ao quadrado, aplicando a esperança e impondo as restrições fica:

$$
E\left(B_{k}^{2}\right)=I J L^{2} \sigma_{a r}^{2}+I^{2} J^{2} L^{2} b_{k}^{2}+I J L^{2} \sigma_{a b r}^{2}+I J L \sigma^{2} \text {. }
$$

Desenvolvendo virá:

$$
\begin{aligned}
\frac{1}{I J L} \sum_{k=2}^{K}\left[E\left(B_{k}^{2}\right)\right]= & L(K-1) \sigma_{a r}^{2}+I J L \sum_{k=2}^{K} b_{k}^{2}+L(K-1) \sigma_{a b r}^{2}+ \\
& +(K-1) \sigma^{2}
\end{aligned}
$$




$$
\begin{aligned}
& B_{1}+y=J L \sum_{i} a_{i}+I L \sum_{j} r_{j}+L \sum_{i j}(a r)_{i j}+I J L b_{1}+ \\
& +J L \sum_{i}(a b)_{i 1}+L \sum_{i j}(a b r)_{i 1 j}+I J \sum_{\ell} c_{\ell}+J \sum_{i \ell}(a c)_{i \ell}+ \\
& +I J \sum_{\ell}(b c)_{1 \ell}+J \sum_{i \ell}(a b c)_{i l \ell}+\sum_{i j l} e_{i j l \ell}+ \\
& +\frac{\sum_{j=2}^{J} e_{1 j 11}}{J-1}+\frac{e_{\ell=2}^{L} e_{111 \ell}}{L-1}-\frac{\sum_{j=2}^{J} \sum_{\ell=2}^{L} e_{1 j 1 \ell}}{(J-1)(L-1)}
\end{aligned}
$$

onde:

$$
\begin{aligned}
\sum_{i j l} e_{i j l l} & \text { é a soma dos (IJL-l) erros associados às } \\
& \text { sub-subparcelas presentes no tratamento } B_{1} \\
& \text { onde ocorreu a perda da observação. Esse } \\
& \text { componente pode ser decomposto da seguinte } \\
& \text { maneira: }
\end{aligned}
$$

$$
\underset{i j \ell}{\sum} e_{i j l l}=\underset{i j l}{\Sigma^{\prime}} e_{i j l \ell}+\sum_{j \ell} e_{i j l \ell}+\sum_{j} e_{1 j 11}
$$

e ainda:

$\underset{i j \ell}{\Sigma^{\prime}} e_{i j l \ell}$ é a soma dos JL(I-1) erros associados às sub-subparcelas dos núcleos $\mathrm{A}_{i} \mathrm{~B}_{k}$, relativos à perda da observação, mas não estão no núcleo $A_{1} B_{1}$ da ocorrêricia de perda, e: 
$\sum_{j \ell} e_{i j l \ell}$ é a soma dos $J(L-1)$ erros associados às su-subparcelas do núcleo $A_{1} B_{1}$, mas não são per tencentes ao tratamento $C_{1}$ onde ocorreu a perda.

Substituindo esses somatórios, elevando ao quadrado, aplicando a esperança e impondo as restrições, fi ca:

$E\left(B_{1}+y\right)^{2}=I^{2} J^{2} L^{2} b_{1}^{2}+I J L^{2} \sigma_{a r}^{2}+I J L^{2} \sigma_{a b r}^{2}+J L(I-1) \sigma^{2}+$

$$
+\frac{J^{2}}{J-1} \sigma^{2}+\frac{\sigma^{2}}{L-1}+J(L-1) \sigma^{2}+\frac{\sigma^{2}}{(J-1)(L-1)}
$$

e daí:

$\frac{E\left(B_{1}+y\right)^{2}}{I J L}=I J L b_{1}^{2}+L \sigma_{a r}^{2}+L \sigma_{a b r}^{2}+\left[1+\frac{K}{I K(J-I)(L-1)}\right] \sigma^{2}$

Substituindo (6), (13) e (14) em (VI), tem- 
E[S.Q.Tratamentos

$$
\begin{aligned}
(B)] & =I J L \sum_{k} b_{k}^{2}+K L \sigma_{a r}^{2}+K L \sigma_{a b r}^{2}+ \\
& +\left[K+\frac{K}{I K(J-1)(L-1)}\right] \sigma^{2}-K L \sigma_{a r}^{2}- \\
& -L \sigma_{a b r}^{2}-\left[1+\frac{1}{I K(J-1)(L-1)}\right] \sigma^{2}
\end{aligned}
$$

Portanto:

$$
\begin{aligned}
& \mathrm{E}[\mathrm{S} \cdot \mathrm{Q} \cdot \text { Tratamentos }(\mathrm{B})]=\mathrm{IJL} \underset{\mathrm{k}}{\sum_{\mathrm{k}}} \mathrm{b}_{\mathrm{l}}^{2}+\mathrm{L}(\mathrm{K}-1) \sigma_{\mathrm{abr}}^{2}+ \\
& +\left[(K-1)+\frac{(K-1)}{\operatorname{IK}(J-1)(L-1)}\right] \sigma^{2},
\end{aligned}
$$

e daí obtém-se:

$$
E[Q \cdot M . T r a t a m e n t o s(B)]=\frac{E[S \cdot Q \cdot \text { Tratamentos }(B)]}{K-1}
$$

ou seja:

$$
\begin{aligned}
E[\text { Q.M.Tratamentos (B) }] & =\frac{I J L}{K-1} \sum b_{k}^{2}+L \sigma_{a b r}^{2}+ \\
& +\left[1+\frac{1}{I K(J-I)(I-I)}\right] \sigma^{2}
\end{aligned}
$$


4.2.7. Interação de Tratamentos $(A \times B)$

$$
\begin{aligned}
E(S . Q . \text { Interação } A x B) & =\frac{1}{J L} E\left[\sum_{i k}\left(A_{i} B_{k}\right)^{2}\right]+\frac{1}{J L} E\left(A_{1} B_{1}+y\right)^{2}- \\
& -E(C)-E[S \cdot Q . \text { Tratamentos (A)] - } \\
& -E[\text { S.Q.Tratamentos (B) }] \quad \text { (VII) }
\end{aligned}
$$

onde :

$$
\begin{aligned}
& A_{i} B_{k} \text { é o total de todas as sub-subparcelas que com- } \\
& \text { põem cada núcleo } A_{i} B_{k} \text { onde não houve a perda da } \\
& \text { observação, e: } \\
& A_{1} B_{1} \text { é o total de todas as sub-subparcelas presentes } \\
& \text { no núcleo } A_{1} B_{1} \text { onde houve a perda. }
\end{aligned}
$$

A representação, através do modelo matemātí co, de cada núcleo $A_{i} B_{k}$, onde não houve perda de informação, é a seguinte:

$$
\begin{aligned}
A_{i} B_{k}=\sum_{j \ell} x_{i j k \ell} & =J L a_{i}+L \sum_{j} r_{j}+L \sum_{j}(a r)_{i j}+J L b_{k}+ \\
& +J L(a b)_{i k}+L \sum_{j}(a b r)_{i k j}+J \sum_{\ell} c_{\ell}+ \\
& +J \sum_{\ell}(a c)_{i \ell}+J \sum_{\ell}(b c)_{k \ell}+J \sum_{\ell}(a b c)_{i k \ell}+ \\
& +\sum_{j \ell} e_{i j k \ell}
\end{aligned}
$$


Elevando ao quadrado, aplicando a esperança e impondo as restrições fica:

$$
\begin{aligned}
E\left(A_{i} B_{k}\right)^{2}=J^{2} L^{2} a_{i}^{2} & +J L^{2} \sigma_{a r}^{2}+J^{2} L^{2} b_{k}^{2}+J^{2} L^{2}(a b) \underset{i k}{2}+J L^{2} \sigma_{a b r}^{2}+ \\
& +J L \sigma^{2}
\end{aligned}
$$

Desenvolvendo em relação a $i$ e $k$ tem-se:

c) para os núcleos $A_{i} B_{k}$ independentes da perda de uni dade:

$\frac{1}{J L} \sum_{i=2}^{I} \sum_{k}\left[E\left(A_{i} B_{k}\right)^{2}\right]=J K L \sum_{i=2}^{I} a_{i}^{2}+K L(I-1) \sigma_{a r}^{2}+J L(I-1) \sum_{k} b_{k}^{2}+$

$$
\begin{aligned}
& +\mathrm{JL} \sum_{i=2}^{I} \sum_{k}(a b) \underset{i k}{2}+K L(I-1) \sigma_{a b r}^{2}+ \\
& +K(I-1) \sigma^{2}
\end{aligned}
$$

d) para os núcleos $A_{1} B_{k}$ independentes da perda da informação:

$$
\begin{aligned}
\frac{1}{\mathrm{JL}} \sum_{k=2}^{K}\left[\mathrm{E}\left(\mathrm{A}_{1} \mathrm{~B}_{k}\right)^{2}\right] & =\mathrm{JL}(\mathrm{K}-1) \mathrm{a}_{1}^{2}+\mathrm{L}(\mathrm{K}-1) \sigma_{\mathrm{ar}}^{2}+\mathrm{JL} \underset{k=2}{\sum_{k=2}^{K}} b_{k}^{2}+ \\
& +\mathrm{JL} \underset{k=2}{\mathrm{~K}}(\mathrm{ab}){ }_{1 \mathrm{k}}^{2}+\mathrm{L}(\mathrm{K}-1) \sigma_{\mathrm{abr}}^{2}+(\mathrm{K}-1) \sigma^{2}
\end{aligned}
$$


Para o núcleo A $\mathrm{B}_{1}$ onde ocorreu a perda tenr -se:

$$
\begin{aligned}
& \mathrm{A}_{1} \mathrm{~B}_{1}+\mathrm{Y}=\mathrm{JL} \mathrm{a}_{1}+\mathrm{L} \sum_{j}^{\sum} \mathrm{r}_{j}+\mathrm{L}_{j} \sum_{j}(\mathrm{ar})_{1 j}+\mathrm{JL} \mathrm{b}_{1}+\mathrm{JL}(\mathrm{ab})_{11}+ \\
& +\mathrm{L} \sum_{j}^{\sum}(\mathrm{abr})_{11 j}+\mathrm{J} \sum_{\ell}^{\sum c_{\ell}}+\mathrm{J} \sum_{\ell}^{\sum}(\mathrm{ac})_{1 \ell}+\mathrm{J} \sum_{\ell}^{\sum(b c)_{1 \ell}}+ \\
& +J \sum_{l}(a b c)_{11 l}+\sum_{j l}^{\prime} e_{1 j 1 l}+\frac{\sum_{j=2}^{J} e_{1 j 11}}{J-1}+ \\
& +\frac{\sum_{\ell=2}^{L} e_{111 \ell}}{L-1}-\frac{\sum_{j=2}^{J} \sum_{=2}^{L} e_{1 j 1 \ell}}{(J-1)(L-1)}
\end{aligned}
$$

onde:

$$
\begin{array}{ll}
\sum^{\prime} e_{1 j \ell l} & \text { é a soma dos }(J L-l) \text { erros associados às sub- } \\
& \text {-subparcelas presentes no núcleo } A_{1} B_{1} \text { onde } \\
& \text { ocorreu a perda. Esses erros podem se decom } \\
& \text { por em: }
\end{array}
$$

$$
\sum_{j \ell}^{\prime} e_{1 j 1 \ell}=\sum_{j \ell}^{\prime \prime} e_{1 j 1 \ell}+\sum_{j=2}^{J} e_{1 j 11}
$$

e,

$$
\begin{aligned}
\sum^{\prime \prime} e_{1 j l \ell} & \text { é a soma dos } \mathrm{J}(\mathrm{L}-1) \text { erros associados às sub- } \\
& \text {-subparcelas presentes no núcleo } \mathrm{A}_{1} \mathrm{~B}_{1} \text { onde } \\
& \text { ocorreu a perda mas não estão nas unidades } \\
& \text { presentes relativas ao tratamento } \mathrm{C}_{1} \text { desse } \\
& \text { núcleo. }
\end{aligned}
$$


Pela substituição desses somatórios e repetindo as operações anteriores chega-se à:

$$
\begin{aligned}
E\left(A_{1} B_{1}+y\right)^{2} & =J^{2} L^{2} a_{1}^{2}+J L^{2} \sigma_{a r}^{2}+J^{2} L^{2} b_{1}^{2}+J^{2} L^{2}(a b)_{11}^{2}+ \\
& +J L^{2} \sigma_{a b r}^{2}+J(L-1) \sigma^{2}+\frac{\sigma^{2}}{L-1}+\frac{J^{2}}{J-1} \sigma^{2}+\frac{\sigma^{2}}{(J-I)(L-1)}
\end{aligned}
$$

e desenvolvendo tem-se:

$$
\begin{aligned}
\frac{E\left(A_{1} B_{1}+Y\right)}{J L}=J L a_{1}^{2} & +L \sigma_{a r}^{2}+J L b_{1}^{2}+J L(a b)_{11}^{2}+L \sigma_{a b r}^{2}+ \\
& +\left[1+\frac{I K}{I K(J-I)(L-I)}\right] \sigma^{2}
\end{aligned}
$$

Pela substituição das expressões obtidas de (III), (VI), (6), (c), (d) e (15) em (VII) virá:

$$
\begin{aligned}
& E(S . Q . \text { Interação } A x B)=J L \sum_{i k}(a b){ }_{i k}^{2}+L(I-1)(K-1) \sigma_{a b r}^{2}+ \\
& +\left[(I-I)(K-1)+\frac{(I-1)(K-1)}{I K(J-I)(L-1)}\right] \sigma^{2}
\end{aligned}
$$

e então:

$$
E(Q . M . \text { Interação } A x B)=\frac{E(S . Q . \text { Interação AxB })}{(I-1)(K-1)}
$$


64.

donde resulta:

$$
\begin{aligned}
& E(Q . M . I n t e r a c ̧ a ̃ o . ~ A x B)=\frac{J L}{(I-I)(K-1)} \sum_{i k}(a b)_{i k}^{2}+I \sigma_{a b r}^{2}+ \\
& +\left[1+\frac{1}{\operatorname{IK}(J-1)(L-1)}\right] \sigma^{2} .
\end{aligned}
$$

4.2.8. Subparcelas

$$
\begin{aligned}
E(S \cdot Q . \text { Subparcelas }) & =\frac{1}{L} E\left[\sum_{i j k}\left(A_{i} B_{k} R_{j}\right)^{2}\right]+ \\
& +\frac{I}{L} E\left(A_{1} B_{1} R_{1}+y\right)^{2}-E(C)
\end{aligned}
$$

onde:

$$
\begin{aligned}
& A_{i} B_{k} R_{j} \text { é o total das sub-subparcelas de cada subpar- } \\
& \text { cela onde não houve perda da informação, e: } \\
& A_{1} B_{1} R_{1} \text { é } \circ \text { total das sub-subparcelas presentes na } \\
& A_{i} B_{k} R_{j} \text { pode ser representado por: } \\
& A_{i} B_{k} R_{j}=\sum_{\ell} x_{i j k \ell}=L a_{i}+L r_{j}+L(a r)_{i j}+L b_{k}+L(a b)_{i k}+ \\
& +L(a b r)_{i k j}+\sum_{\ell} c_{\ell}+\sum_{\ell}(a c)_{i \ell}+\sum_{\ell}(b c)_{k \ell}+\sum_{\ell}(a b c) i k \ell+ \\
& +\sum_{\ell} e_{i j k \ell} .
\end{aligned}
$$


Por procedimentos análogos aos anteriormente aplicados chega-se à:

$$
\begin{gathered}
E\left(A_{i} B_{k} R_{j}\right)^{2}=L^{2} a_{i}^{2}+L^{2} r_{j}^{2}+L^{2} \sigma_{a r}^{2}+L^{2} b_{k}^{2}+L^{2}(a b)_{i k}^{2}+ \\
+L^{2} \sigma_{a b r}^{2}+L \sigma^{2} .
\end{gathered}
$$

Desenvolvendo para as (IJK - I) combinações

tem-se:

e) para as subparcelas $A_{i} B_{k} R_{j}$ independentes de $A_{1}$ : $\frac{1}{L} \sum_{i=2}^{I} \sum_{j k}\left[E\left(A_{i} B_{k} R_{j}\right)^{2}\right]=J K L \sum_{i=2}^{I} a_{i}^{2}+K L(I-1) \sum_{j} r_{j}^{2}+$ $+J K L(I-1) \sigma_{a r}^{2}+J L(I-1) \sum_{k} b_{k}^{2}+$ $+\mathrm{JL} \sum_{i=2}^{I} \sum_{k}(a b)_{i k}^{2}+J K L(I-I) \sigma_{a b r}^{2}+$ $+\mathrm{JK}(I-1) \sigma^{2}$

f) para as subparcelas $A_{l} B_{k} R_{j}$ independentes de $B_{l}$ :

$$
\begin{aligned}
\frac{1}{L} \sum_{k=2}^{K} \sum_{j}\left[E\left(A_{1} B_{k} R_{j}\right)^{2}\right] & =J L(K-1) a_{1}^{2}+L(K-1) \sum_{j} r_{j}^{2}+J L(K-1) \sigma_{a r}^{2}+ \\
& \left.+J L \sum_{k=2}^{K} b_{k}^{2}+J L \underset{k=2}{\sum_{k}^{K}(a b)}\right)_{1 k}^{2}+ \\
& +J L(K-1) \sigma_{a b r}^{2}+J(K-1) \sigma^{2} \quad \text { (f) }
\end{aligned}
$$


g) para as subparcelas $A_{1} B_{1} R_{j}$, exceto $A_{1} B_{1} R_{1}$ onde 으 correu a perda:

$$
\begin{aligned}
\frac{1}{L} \sum_{j=2}^{J}\left[E\left(A_{1} B_{1} R_{j}\right)^{2}\right] & =L(J-1) a_{1}^{2}+L \underset{j=2}{J} r_{j}^{2}+L(J-1) \sigma_{a r}^{2}+ \\
& +L(J-1) b_{1}^{2}+L(J-1)(a b)_{11}^{2}+L(J-1) \sigma_{a b \dot{r}}^{2}+ \\
& +(J-1) \sigma^{2}
\end{aligned}
$$

Para a subparcela'relativa à perda de obser

vação virá:

$$
\begin{aligned}
& \left(A_{1} B_{1} R_{1}+y\right)=L a_{1}+L r_{1}+L(a r)_{11}+L b_{1}+L(a b)_{11}+ \\
& +L(a b r)_{111}+\sum_{\ell} c_{\ell}+\sum_{\ell}(a c)_{j} 1 \ell+\sum_{\sum}(b c)_{1 \ell}+ \\
& +\sum_{\ell}(a b c)_{11 \ell}+\sum_{\ell} e_{111 \ell}+\frac{\sum_{j=2} e_{1 j 11}}{J-1}+ \\
& +\frac{\sum_{\ell=2}^{L} e_{111 \ell}}{L-1}-\frac{\sum_{j=2}^{J} \sum_{l=2}^{L} e_{1 j 1 \ell}}{(J-1)(L-1)}
\end{aligned}
$$

e então:

$$
\begin{aligned}
E\left(A_{1} B_{1} R_{1}+y\right)^{2} & =L^{2} a_{1}^{2}+L^{2} r_{1}^{2}+L^{2} \sigma_{a r}^{2}+L^{2} b_{1}^{2}+L^{2}(a b)_{11}^{2}+ \\
& +L^{2} \sigma_{a b r}^{2}+\frac{L^{2}}{L-1} \sigma^{2}+\frac{\sigma^{2}}{J-1}+\frac{\sigma^{2}}{(J-1)(L-1)}
\end{aligned}
$$


67.

donde :

$$
\begin{aligned}
\frac{E\left(A_{1} B_{1} R_{1}+y\right)^{2}}{L} & =L a_{1}^{2}+L r_{1}^{2}+L \sigma_{a r}^{2}+L b_{1}^{2}+L(a b)_{11}^{2}+ \\
& +L \sigma_{a b r}^{2}+\left[1+\frac{I J K}{I K(J-I)(L-1)}\right] \sigma^{2}
\end{aligned}
$$

Substituindo (6), (e), (f), (g) e (16) em

(VIII) tem-se:

$E(S . Q$. Subparcelas $)=J K L \sum_{i} a_{i}^{2}+\operatorname{IKL} \sum_{j} r_{j}^{2}+K L(I J-I) \sigma_{a r}^{2}+$

$$
\begin{aligned}
& +I J L \underset{k}{\sum_{k} b_{k}^{2}}+J L \underset{i k}{\sum_{i k}(a b){ }_{i k}^{2}}+L(I J K-1) \sigma_{a b r}^{2}+ \\
& +\left[(I J K-1)+\frac{(I J K-1)}{I K(J-1)(L-1)}\right] \sigma^{2} .
\end{aligned}
$$

4.2.9. Residuo (b)

$E[$ S.Q.Resíduo (b) $]=E(S . Q . S u b p a r c e l a s)-E(S . Q . P a r c e l a s)-$

- E[S.Q.Tratamentos (B)] -

- E(S.Q.Interação AxB)

(IX) 
Substituindo as expressões obtidas de (IV), (VI), (VII) e (VIII) em (IX) obtem-se:

$$
\begin{aligned}
E[S \cdot Q \cdot \operatorname{Residuo~(b)]} & =\operatorname{IL}(J-1)(K-1) \sigma_{\mathrm{abr}}^{2}+ \\
+ & +\left[I(J-1)(K-1)+\frac{I(J-1)(K-1)}{I K(J-1)(L-1)}\right] \sigma^{2} .
\end{aligned}
$$

e a expressão da E[Q.M.Resíduo (b)] dada por:

$$
E[Q \cdot M . R e s i d u o(b)]=L \sigma_{a b r}^{2}+\left[1+\frac{1}{I K(J-1)(L-1)}\right] \sigma^{2}
$$

4.2.10. Tratamento (C)

$$
\begin{aligned}
& E[S . Q . T r a t a m e n t o s \text { (C) }]=\frac{1}{I J K} E\left[\begin{array}{ll}
L & C_{\ell=2}^{2} \\
\ell
\end{array}\right]+ \\
& +\frac{1}{I J K} E\left(C_{1}+y\right)^{2}-E(C)
\end{aligned}
$$

onde:

$C_{\ell}$ é a soma das sub-subparcelas associadas a cada tra tamento $C_{\ell}$ e que não estão relacionadas com a perda da observação, e:

$C_{1}$ é a soma das sub-subparcelas presentes e associadas ao tratamento $C_{1}$ onde ocorreu a perda da ob servação. 


$$
\begin{aligned}
& \text { Para cada } C_{\ell} \text { tem-se: } \\
& C_{\ell}=\underset{i j k}{\sum} x_{i j k \ell}=J K \sum_{i}^{\sum} a_{i}+I K \sum_{j} r_{j}+K \sum_{i j}(a r)_{i j}+I J \underset{k}{\sum} b_{k}+ \\
& +J \sum_{i k}(a b)_{i k}+\sum_{i j k}(a b r)_{i k j}+I J K c_{\ell}+ \\
& +J K \sum_{i}(a c)_{i \ell}+I J \sum_{k}(b c)_{k \ell}+J \sum_{i k}(a b c)_{i k \ell}+ \\
& +\sum_{i j k} e_{i j k l} \\
& \text { Por procedimentos análogos aos anteriores }
\end{aligned}
$$

chega-se à:

$$
E\left(C_{\ell}^{2}\right)=I J K^{2} \sigma_{a r}^{2}+I J K \sigma^{2} a b r+I^{2} J^{2} K^{2} c_{l}^{2}+I J K \sigma^{2}
$$

e assim:

$$
\begin{aligned}
& \frac{1}{I J K} E\left[\begin{array}{cc}
L & C_{l}^{2} \\
l=2 & l
\end{array}\right]=K(L-1) \sigma_{a r}^{2}+(L-1) \sigma_{a b r}^{2}+I J K \sum_{l=2}^{L} c_{l}^{2}+ \\
& +(L-1) \sigma^{2}
\end{aligned}
$$

Para o tratamento onde ocorreu a perda, o modelo matemático representativo é: 


$$
\begin{aligned}
& C_{1}+y=J K \sum_{i} a_{i}+I K \sum_{j} r_{j}+K \sum_{i j}(a r)_{i j}+I J \underset{k}{\sum} b_{k}+ \\
& +J \sum_{i k}(a b)_{i k}+\sum_{i j k}(a b r)_{i k j}+I J K c_{1}+J K \sum_{i}^{\sum(a c)_{i 1}}+ \\
& +I J \sum_{k}^{\sum}(b c)_{k 1}+J \sum_{i k}(a b c)_{i k 1}+\sum_{i j k} e_{i j k 1}+\frac{\sum_{j=2}^{J} e_{1 j 11}}{J-1}+ \\
& +\frac{\sum_{\ell=2}^{L} e_{111 \ell}}{L-1}-\frac{\sum_{j=2}^{J} \sum_{=2}^{L} e_{1 j 1 \ell}}{(J-1)(L-1)}
\end{aligned}
$$

onde:

$$
\begin{aligned}
& \sum_{i j k} e_{i j k l} \text { é a soma dos (IJK-l) erros associados às } \\
& \text { sub-subparcelas relativas ao tratamento } c_{1} \\
& \text { onde ocorreu a perda da observação. Esse } \\
& \text { componente pode ser desdobrado em: } \\
& \underset{i j k}{\sum} e_{i j k l}=\underset{i j k}{\sum^{\prime} e_{i j k l}+\sum_{j k}^{\prime} e_{1 j k l}+\sum_{j} e_{1 j l 1}}
\end{aligned}
$$

e ainda:

$\underset{i j k}{\Sigma^{\prime}} e_{i j k l}$ sub-subparcelas do tratamento $C_{1}$, onde ocorreu a perda, mas não estão no núcleo $\mathrm{A}_{1} \mathrm{~B}_{1}$ correspondente à perda, e: 
71.

$\sum_{j k}^{\prime} e_{1 j k_{1}}$ é a soma dos $\mathrm{J}(\mathrm{K}-1)$ erros associados às sub-subparcelas do tratamento $C_{1}$, onde houve a perda, receberam o tratamento $A_{1}$ mas não receberam o tratamento $B_{1}$ relativo à perda da informação.

Pela substituição desses somatórios, e por processos análogos aos anteriores tem-se:

$E\left(C_{1}+y\right)^{2}=I J K^{2} \sigma_{a r}^{2}+I J K \sigma_{a b r}^{2}+\left(I^{2} J^{2} K^{2} c_{1}^{2}+\frac{\sigma^{2}}{L-1}+J K(I-I) \sigma^{2}+\right.$

$$
+\mathrm{J}(\mathrm{K}-1) \sigma^{2}+\frac{\mathrm{J}^{2}}{\mathrm{~J}-1} \sigma^{2}+\frac{\sigma^{2}}{(\mathrm{~J}-1)(\mathrm{L}-1)}
$$

e assim:

$\frac{E\left(C_{1}+y\right)^{2}}{I J K}=K \sigma_{a r}^{2}+\sigma_{a b r}^{2}+I J K c_{1}^{2}+\left[1+\frac{L}{I K(J-1)(L-I)}\right] \sigma^{2}$

Substituindo (6), (17) e (18) em (X) virá:

$E[$ S.Q.Tratamentos $(C)]=\operatorname{IJK} \underset{l}{\sum_{l}} c_{\ell}^{2}+\left[(L-1)+\frac{(L-1)}{\operatorname{IK}(J-I)(L-I)}\right] \sigma^{2}$ 


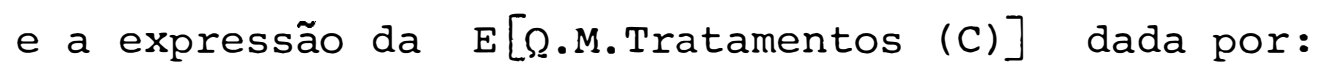

$E[Q . M . T r a t a m e n t o s(C)]=\frac{I J K}{I-1} \sum_{\ell} c_{\ell}^{2}+\left[1+\frac{1}{I K(J-1)(L-1)}\right] \sigma^{2}$.

4.2.11. Interação dos Tratamentos ( $A \times C)$

$E(S . Q$. Interação $A x C)=\frac{1}{J K} E\left[\sum_{i \ell}\left(A_{i} C_{\ell}\right)^{2}\right]+\frac{1}{J K} E\left(A_{1} C_{1}+y\right)^{2}-$

- E(C) - E[S.Q.Tratamentos (A)] -

- E[S.Q.Tratamentos (C)]

$(\mathrm{XI})$

onde:

$A_{i} C_{\ell}$ é o total das sub-subparcelas associadas a cada combinação dos tratamentos $A_{i} C_{\ell}$, que não se re fere à perda da observação, e:

$A_{1} C_{1}$ é $O$ total das sub-subparcelas presentes na combinação dos tratamentos $A_{1} C_{1}$ onde ocorreu a per da de sub-subparcela.

Cada total $\mathrm{A}_{i} \mathrm{C}_{\ell}$ pode ser representado, segundo o modelo matemático, da seguinte forma: 


$$
\begin{aligned}
A_{i} c_{\ell}=\sum_{j k} x_{i j k l} & =J k a_{i}+k \sum_{j} r_{j}+k \sum_{j}(a r)_{i j}+J \sum_{k} b_{k}+ \\
& +J \sum_{k}(a b)_{i k}+\sum_{j k}(a b r)_{i k j}+J k c_{\ell}+J k(a c)_{i l}+ \\
& +J \sum_{k}(b c)_{k \ell}+J \sum_{k}^{\sum(a b c)_{i k \ell}}+\sum_{j k} e_{i j k l}
\end{aligned}
$$

cuja expressão da esperança do quadrado é:

$$
\begin{aligned}
E\left(A_{i} C_{\ell}\right)^{2}= & J^{2} K^{2} a_{i}^{2}+J K^{2} \sigma_{a r}^{2}+J K \sigma_{a b r}^{2}+J^{2} K^{2} c_{\ell}^{2}+ \\
& +J^{2} K^{2}(a c)_{i \ell}^{2}+J K \sigma^{2}
\end{aligned}
$$

Desenvolvendo o somatório em relação a $j$ e $\underline{k}$ tem-se:

h) para os totais $A_{i} C_{\ell}$ independentes de $A_{1}$ :

$$
\begin{aligned}
& \frac{1}{J K} \sum_{i=2}^{I} \sum\left[E\left(A_{i} C_{\ell}\right)^{2}\right]=J K L \sum_{i=2}^{I} a_{i}^{2}+K L(I-I) \sigma_{a r}^{2}+L(I-I) \sigma_{a b r}^{2}+ \\
& +\mathrm{JK}(\mathrm{I}-1) \sum_{\ell} \mathrm{c}_{\ell}^{2}+\mathrm{JK} \underset{i=2}{\mathrm{I}} \sum_{\ell}(\mathrm{ac})_{\mathrm{i} \ell}^{2}+ \\
& +L(I-1) \sigma^{2}
\end{aligned}
$$


m) para os totais $\mathrm{A}_{1} \mathrm{C}_{\ell}$ independentes de $\mathrm{C}_{1}$ :

$$
\begin{aligned}
\frac{1}{J K} \sum_{\ell=2}^{L}\left[E\left(A_{1} C_{l}\right)^{2}\right] & =J K(L-1) a_{1}^{2}+K(L-1) \sigma_{a r}^{2}+(L-1) \sigma_{a b r}^{2}+ \\
& +J K \sum_{\ell=2}^{L} c_{l}^{2}+J K \sum_{\ell=2}^{L}(a c)_{l \ell}+(L-1) \sigma^{2} \quad(m)
\end{aligned}
$$

Para $\circ$ total $A_{1} C_{1}$. relativo à perda de observação tem-se:

$$
\begin{aligned}
& A_{1} C_{1}+Y=J K a_{1}+K \sum_{j} r_{j}+K \sum_{j}(a r)_{1 j}+J \sum_{k} b_{k}+J \sum_{k}(a b)_{1 k}+\underset{j k}{\sum}(a b r)_{1 k j}+ \\
& +J K c_{1}+J K(a c)_{11}+J \sum_{k}^{\sum}(b c)_{k 1}+J \sum_{k}^{\sum(a b c)_{1 k_{1}}}+\sum_{j k}^{\sum} e_{1 j k_{1}}+ \\
& +\frac{\sum_{j=2}^{J} e_{1 j 11}}{J-1}+\frac{\sum_{\ell=2}^{L} e_{111 \ell}}{L-1}-\frac{\sum_{j=2}^{J} \sum_{=2}^{L} e_{1 j 1 \ell}}{(J-1)(I-1)}
\end{aligned}
$$

onde:

$$
\begin{aligned}
& \sum_{j k} e_{1 j k 1} \text { è a soma dos }(J k-1) \text { erros associados às sub- } \\
& \text { - subparcelas da combinação dos tratamentos } \\
& A_{1} C_{1} \text { onde ocorreu a perda da informação. Es. . } \\
& \text { se componente pode ser desdobrado em: } \\
& \sum_{j k} e_{1 j k 1}=\sum_{j k}^{\prime} e_{1 j k 1}+\underset{j=2}{J} e_{1 j 11} .
\end{aligned}
$$


75.

Substituindo esses somatórios, elevando ao quadrado, aplicando a esperança e impondo as restrições fica:

$E\left(A_{1} C_{1}+y\right)^{2}=J^{2} K^{2} a_{1}^{2}+J K^{2} \sigma_{a r}^{2}+J K \sigma_{a b r}^{2}+J^{2} K^{2} c_{i}^{2}+$

$$
\begin{aligned}
& +J^{2} K^{2}(a c)_{11}^{2}+J(K-1) \sigma^{2}+\frac{J^{2}}{J-1} \sigma^{2}+\frac{\sigma^{2}}{L-1}+ \\
& +\frac{\sigma^{2}}{(J-1)(L-1)}
\end{aligned}
$$

e então:

$$
\begin{aligned}
\frac{E\left(A_{1} C_{1}+y\right)^{2}}{J K} & =J K a_{1}^{2}+K \sigma_{a r}^{2}+\sigma_{a b r}^{2}+J K c_{1}^{2}+J K(a c)_{11}^{2}+ \\
& +\left[1+\frac{I L}{I K(J-1)(L-1)}\right] \sigma^{2}
\end{aligned}
$$

Substituindo as expressões das esperanças das somas de quadrados, resultantes de (III), (VI), (6) , (h), (m) e (19) em (XI) virá:

$$
\begin{aligned}
E(S . Q . \text { Interação } \mathrm{AxC}) & =\mathrm{JK} \sum_{i \ell}(\mathrm{ac})_{i \ell}^{2}+ \\
& +\left[(I-I)(I-1)+\frac{(I-1)(I-1)}{I K(J-1)(I-1)}\right] \sigma^{2}
\end{aligned}
$$


e a correspondente expressão da E(Q.M.Interação $\mathrm{AxC}$ ) dada por:

$$
\begin{aligned}
& E(Q . M . I n t e r a c ̧ a ̃ o ~ A x C)=\frac{J K \sum_{i \ell}(a c)_{i l}^{2}}{\left(I-I^{\prime}\right)(L-1)}+ \\
& +\left[1+\frac{1}{\operatorname{IK}(J-1)(L-1)}\right] \sigma^{2} .
\end{aligned}
$$

4.2.12. Interação dos Tratamentos $(B \times C)$

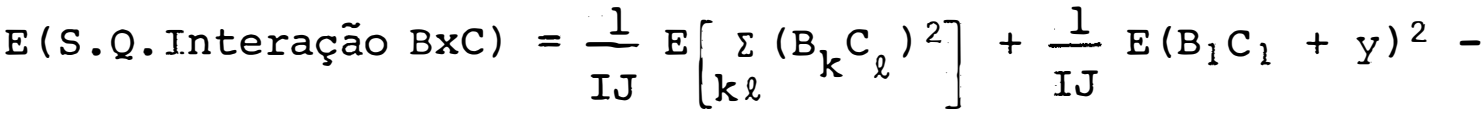

$$
\begin{aligned}
& \text { - E(C) - E[S.Q.Tratamentos (B)]- } \\
& \text { - E[S.Q.Tratamentos (C)] }
\end{aligned}
$$

onde:

$\mathrm{B}_{\mathrm{k}} \mathrm{C}_{\ell}$ é $\mathrm{O}$ total de todas as sub-subparcelas que compõem cada combinação dos tratamentos $B_{k} C_{\ell}$, ex ceto daquela referente à perda da observação, e:

$B_{1} C_{1}$ é 0 total de todas as sub-subparcelas presentes na combinação dos tratamentos $B_{1} C_{1}$ relativo à perda da informação. 
Cada $B_{k} C_{l}$ pode ser representado por:

$$
\begin{aligned}
B_{k} C_{\ell}= & \sum_{i j} x_{i j k \ell}=J \sum_{i} a_{i}+I \sum_{j} r_{j}+\sum_{i j}(a r)_{i j}+I J b_{k}+ \\
& +J \sum_{i}(a b)_{i k}+\sum_{i j}(a b r)_{1 k j}+I J c_{\ell}+J \sum_{i}(a c)_{i \ell}+ \\
& +I J(b c)_{k \ell}+J \sum_{i}(a b c)_{i k \ell}+\sum_{i j} e_{i j k \ell}
\end{aligned}
$$

e assim:

$$
\begin{aligned}
E\left(B_{k} C_{\ell}\right)^{2}=I J \sigma_{a r}^{2} & +I^{2} J^{2} b_{k}^{2}+I J \sigma_{a b r}^{2}+I^{2} J^{2} c_{\ell}^{2}+I^{2} J^{2}(b c)_{k \ell}^{2}+ \\
& +I J \sigma^{2} .
\end{aligned}
$$

Desenvolvendo e isolando os núcleos pertinentes virá:

n) para os totais $\left(B_{k} C_{\ell}\right)$ excluindo o da combinação $\mathrm{B}_{1} \mathrm{C}_{1}$ onde ocorreu a perda:

$$
\begin{aligned}
\frac{1}{I J} \sum_{k=2}^{K} \sum_{\ell=2}^{L}\left[E\left(B_{k} C_{\ell}\right)^{2}\right] & =(K-1)(L-1) \sigma_{a r}^{2}+I J(L-1) \sum_{k=2}^{K} b_{k}^{2}+ \\
& +(K-1)(L-1) \sigma_{a b r}^{2}+I J(K-1) \sum_{\ell=2}^{L} c_{\ell}^{2}+ \\
& +I J \sum_{k=2}^{K} \sum_{\ell=2}^{L}(b c)_{k \ell}^{2}+(K-1)(L-1) \sigma^{2}
\end{aligned}
$$


78.

o) para os totais de $\left(B_{1} C_{\ell}\right)$ isentos de $C_{1}$ :

$$
\begin{aligned}
& \frac{1}{I J} \sum_{\ell=2}^{L}\left[E\left(B_{1} C_{\ell}\right)^{2}\right]=(L-1) \sigma_{a r}^{2}+I J(L-I) b_{1}^{2}+(L-1) \sigma_{a b r}^{2}+ \\
& + \text { IJ } \sum_{\ell=2}^{L} c_{\ell}^{2}+I J \sum_{\ell=2}^{L}(b c)_{1 \ell}^{2}+(L-1) \sigma^{2} \\
& \text { p) para os totais de }\left(B_{k} C_{1}\right) \text { isentos de } B_{1} \text { : } \\
& \frac{I}{I J} \sum_{k=2}^{K}\left[E\left(B_{k} C_{1}\right)^{2}\right]=(K-1) \sigma_{a r}^{2}+I J \sum_{k=2}^{K} b_{k}^{2}+(K-1) \sigma_{a b r}^{2}+ \\
& +I J(K-1) c_{1}^{2}+I J \sum_{k=2}^{K}(b c)_{k 1}^{2}+(K-1) \dot{\sigma}^{2} \quad(p)
\end{aligned}
$$

$$
\text { Para o total } B_{1} C_{1} \text { onde ocorreu a perda } f_{\underline{i}}
$$

ca:

$$
\begin{aligned}
B_{1} C_{1}+y & =J \sum_{i} a_{i}+I \sum_{j} r_{j}+\sum_{i j}(a r)_{i j}+I J b_{1}+J \sum_{i}(a b)_{i 1}+ \\
& +\sum_{i j}(a b r)_{i 1 j}+I J c_{1}+J \sum_{i}(a c)_{i 1}+I J(b c)_{11}+ \\
& +J \sum_{i}(a b c)_{i 11}+\sum_{i j} e_{i j 11}+\frac{e_{1 j 11}}{J-1}+ \\
& +\frac{\sum \sum_{l=2} e_{111 \ell}}{L-1}-\frac{\sum_{j=2}^{J} \sum_{\ell=2}^{L} e_{1 j 1 . \ell}}{(J-1)(L-1)}
\end{aligned}
$$


onde:

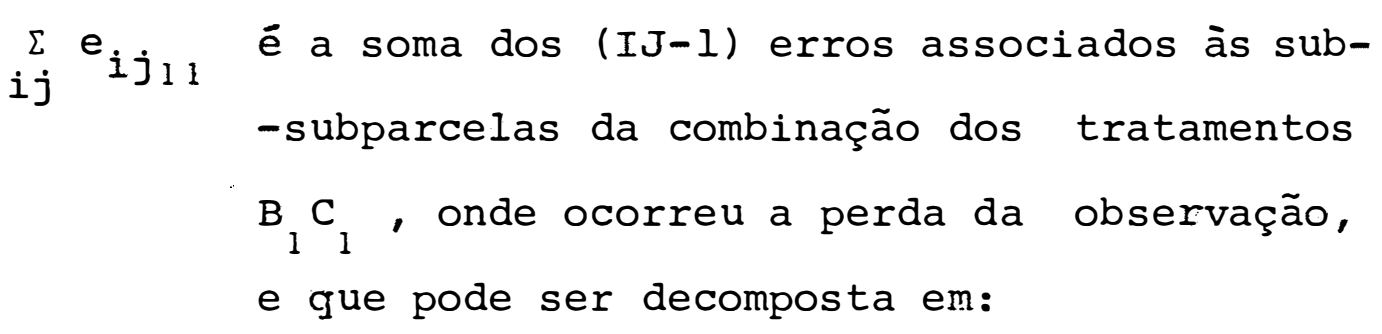

$$
\sum_{i j} e_{i j 11}=\sum_{i j}^{\prime} e_{i j 11}+\sum_{j=2}^{J} e_{1 j 11}
$$

onde, a $\sum_{i j}^{\prime} e_{i j l l}$ estão associados $J(I-l)$ erros provenientes das sub-subparcelas relativas à combinação $B_{1} C_{1}$ mas não receberam o tratamento $A_{1}$ relativo à unidade perdida. Pela substituição dos somatórios e pela aplicação da esperança ao quadrado dessa expressão obtém-se: $E\left(B_{1} C_{1}+y\right)^{2}=I J \sigma_{a r}^{2}+I^{2} J^{2} b_{1}^{2}+I J \underset{a b r}{\sigma^{2}}+I^{2} J^{2} c_{1}^{2}+$

$$
\begin{aligned}
& +I^{2} J^{2}(b c)_{11}^{2}+\frac{\sigma^{2}}{I-1}+\frac{J^{2} \sigma^{2}}{J-1}+J(I-1) \sigma^{2}+ \\
& +\frac{\sigma^{2}}{(J-1)(L-1)}
\end{aligned}
$$

e daí: 


$$
\begin{gathered}
\frac{E\left(B_{1} C_{1}+y\right)^{2}}{I J}=\sigma_{a r}^{2}+I J b_{1}^{2}+\sigma_{a b r}^{2}+I J c_{1}^{2}+I J(b c)_{11}^{2}+ \\
+\left[1+\frac{K L}{I K(J-I)(L-I)}\right] \sigma^{2}
\end{gathered}
$$

Substituindo em (XII) as expressões das es peranças das somas de quadrados resultantes de (VI) e (X) a lém das expressões de $(6),(n),(0),(p)$ e (20) resulta:

$$
\begin{aligned}
& \mathrm{E}(\mathrm{S} \cdot \mathrm{Q} \text {. Interação } \mathrm{BxC})=\mathrm{IJ} \underset{\mathrm{k} \ell}{\sum(\mathrm{bc})_{\mathrm{k} \ell}^{2}}+ \\
& +\left[(K-1)(L-1)+\frac{(K-1)(L-1)}{I K(J-1)(L-1)}\right] \sigma^{2}
\end{aligned}
$$

e a expressão do quadrado médio correspondente será:

$$
\begin{aligned}
& E(Q . M . I n t e r a c ̧ a ̃ o ~ B x C)=\frac{I J \underset{k \ell}{\sum}(b c)_{k \ell}^{2}}{(K-1)(L-1)}+ \\
& +\left[1+\frac{1}{\operatorname{IK}(J-1)(L-1)}\right] \sigma^{2} .
\end{aligned}
$$


4.2.13. Interaç̃o dos Tratamentos $(A \times B \times C)$

$$
\begin{aligned}
& E(S . Q . I n t e r a c ̧ a ̃ o ~ A x B x C)=\frac{I}{J} E\left[\sum_{i k \ell}\left(A_{1} \cdot B_{k} C_{\ell}\right)^{2}\right]+\frac{1}{J} E\left(A_{1} B_{1} C_{1}+y\right)^{2}-E(C)- \\
& \text { - E[S.Q.Tratamentos (A)] - E[S.Q.Tratamentos (B)] - } \\
& \text { - E[S.Q.Tratamentos (C)] - E(S.Q.Interação AxB) - } \\
& \text { - E(S.Q.Interação AxC) - E (S.@.Interação BxC) }
\end{aligned}
$$

onde:

$\mathrm{A}_{i} \mathrm{~B}_{k} \mathrm{C}_{\ell}$ é a soma das sub-subparcelas que compõem cada total da combinação dos tratamentos $\mathrm{A}_{i} \mathrm{~B}_{\mathrm{k}} \mathrm{C}_{l}$, onde não houve unidade perdida, e:

$A_{1}^{B} C_{1}$ é a soma das sub-subparcelas presentes na associação dos tratamentos $\mathrm{A}_{1} \mathrm{~B}_{1} \mathrm{C}_{1}$ relativo a per da da observação.

Através do modelo matemātico, cada total $A_{i} B_{k} C_{l}$ pode ser expresso por: 


$$
\begin{aligned}
A_{i} B_{k} C_{\ell}=\sum_{j} x_{i j k \ell} & =J a_{i}+\sum_{j} r_{j}+\sum_{j}(a r)_{i j}+J b_{k}+J(a b)_{i k}+ \\
& +\sum_{j}(a b r)_{i k j}+J c_{\ell}+J(a c)_{i \ell}+J(b c)_{k \ell}+ \\
& \cdot J(a b c)_{i k \ell}+\sum_{j} e_{i j k \ell}
\end{aligned}
$$

Aplicando a esperança ao quadrado dessa expressão e impondo as restrições fica:

$$
\begin{aligned}
E\left(A_{i} B_{k} C_{\ell}\right)^{2} & =J^{2} a_{i}^{2}+J \sigma_{a r}^{2}+J^{2} b_{k}^{2}+J^{2}(a b)_{i k}^{2}+J \sigma_{a b r}^{2}+ \\
& +J^{2} c_{\ell}^{2}+J^{2}(a c){ }_{i \ell}^{2}+J^{2}(b c)_{k \ell}^{2}+J^{2}(a b c){ }_{i k \ell}^{2}+J \sigma^{2} .
\end{aligned}
$$

Assim tem-se:

q) para cada total $A_{i} B_{k} C_{\ell}$ excluindo a participação do tratamento $\mathrm{A}_{1}$ :

$$
\begin{aligned}
& \frac{1}{J} \sum_{i=2}^{I} \sum_{k \ell}\left[E\left(A_{1} B_{k} C_{\ell}\right)^{2}\right]=J \pi \sum_{i=2}^{I} a_{i}^{2}+K L(I-1) \sigma_{a r}^{2}+J L(I-1) \sum_{k} b_{k}^{2}+ \\
& +\pi \sum_{i=2}^{I} \sum_{k}(a b){ }_{i k}^{2}+K L(I-1) \sigma_{a b r}^{2}+J K(I-1) \sum_{l} c_{l}^{2}+ \\
& +\mathrm{JK} \sum_{i=2}^{I} \sum_{\ell}(\mathrm{ac})_{i \ell}^{2}+\mathrm{J}(\mathrm{I}-1) \sum_{\mathrm{k} \ell}(\mathrm{bc})_{\mathrm{k} \ell}^{2}+ \\
& +\mathrm{J} \sum_{i=2}^{I} \sum_{k \ell}(a b c)_{i k \ell}^{2}+k L(I-1) \sigma^{2} \quad \text { (q) }
\end{aligned}
$$


r) para cada total $A_{1} B_{k} C_{l}$ excluindo a participação do tratamento $B_{1}$ :

$$
\begin{aligned}
& \frac{1}{J} \sum_{k=2}^{K} \sum_{l}\left[E\left(A_{1} B_{k} C_{l}\right)^{2}\right]=\pi(k-1) a_{1}^{2}+L(k-1) \sigma_{a r}^{2}+J \sum_{k=2}^{K} b_{k}^{2}+ \\
& +\pi \cdot \sum_{k=2}^{K}(a b){ }_{1 k}^{2}+L(K-1) \sigma_{a b r}^{2}+J(K-1) \sum_{\ell} c_{l}^{2}+ \\
& +\mathrm{J}(\mathrm{K}-1) \sum_{\ell}(\mathrm{ac})_{1 \ell}^{2}+\mathrm{J} \sum_{\mathrm{k}=2}^{\mathrm{K}} \sum_{\ell}(\mathrm{bc})_{\mathrm{k} \ell}^{2}+ \\
& +J \sum_{k=2}^{K} \sum_{\ell}(a b c)_{1 k \ell}^{2}+L(K-1) \sigma^{2}
\end{aligned}
$$

s) para cada total $A_{1} B_{1} C_{\ell}$ excluindo a participação do tratamento $\mathrm{C}_{1}$ :

$$
\begin{aligned}
\frac{1}{J} \sum_{\ell=2}^{L}\left[E\left(A_{1} B_{1} C_{\ell}\right)^{2}\right] & =J(L-1) a_{1}^{2}+(L-1) \sigma_{a r}^{2}+J(L-1) b_{1}^{2}+J(I-1)(a b)_{11}^{2}+ \\
& +(L-1) \sigma_{a b r}^{2}+J \sum_{\ell=2}^{L} c_{l}^{2}+J \sum_{l=2}^{L}(a c)_{1 \ell}^{2}+ \\
& +J \sum_{\ell=2}^{L}(b c)_{1 \ell}^{2}+J \sum_{\ell=2}^{L}(a b c)_{11 \ell}^{2}+(I-1) \sigma^{2}
\end{aligned}
$$

Para a combinação de tratamentos $A_{1} B_{1} C_{1}$, incluindo a unidade perdida, virá: 


$$
\begin{aligned}
A_{1} B_{1} C_{1}+y & =J a_{1}+\sum_{j} r_{j}+\sum_{j}(a r)_{1 j}+J b_{1}+J(a b)_{11}+\sum_{j}(a b r)_{11 j}+ \\
& +J c_{1}+J(a c)_{11}+J(b c)_{11}+J(a b c)_{111}+\sum_{j} e_{1 j 11}+ \\
& \frac{J}{J} e_{1 j 11}+\frac{\sum_{\ell=2} e_{111 \ell}}{L-1}-\frac{\sum_{j=2} \sum_{\ell=2} e_{1 j 1 \ell}}{(J-1)(L-1)}
\end{aligned}
$$

Aplicando a esperança ao quadrado dessa ex pressão e impondo as restrições virá:

$E\left(A_{1} B_{1} C_{1}+y\right)^{2}=J^{2} a_{1}^{2}+J \sigma_{a r}^{2}+J^{2} b_{1}^{2}+J^{2}(a b)_{11}^{2}+J \sigma_{a b r}^{2}+$

$$
\begin{aligned}
& +J^{2} c_{1}^{2}+J^{2}(a c)_{11}^{2}+J^{2}(b c)_{11}^{2}+J^{2}(a b c)_{111}^{2}+ \\
& +\frac{J^{2}}{J-1} \sigma^{2}+\frac{\sigma^{2}}{L-1}+\frac{\sigma^{2}}{(J-1)(L-1)}
\end{aligned}
$$

e daí:

$$
\begin{aligned}
\frac{E\left(A_{1} B_{1} C_{1}+y\right)^{2}}{J} & =J a_{1}^{2}+\sigma_{a r}^{2}+J b_{1}^{2}+J(a b)_{11}^{2}+\sigma_{a b r}^{2}+J c_{1}^{2}+ \\
& +J(a c)_{11}^{2}+J(b c)_{11}^{2}+J(a b c)_{111}^{2}+ \\
& +\left[1+\frac{I K L}{I K(J-I)(L-1)}\right] \sigma^{2}
\end{aligned}
$$


Pela substituição das expressões das esperanças das somas de quadrados obtidas anteriormente e das expressões (6), (q), (r), (s) e (21) em (XIII) chega-se à:

$E(S . Q$. Interação $A \times B \times C)=J \sum_{i k \ell}(a b c) \underset{i k \ell}{2}+$

$$
+\left[(I-1)(K-1)(L-1)+\frac{(I-1)(K-1)(I-1)}{\operatorname{IK}(J-1)(I-1)}\right] \sigma^{2}
$$

e ainda:

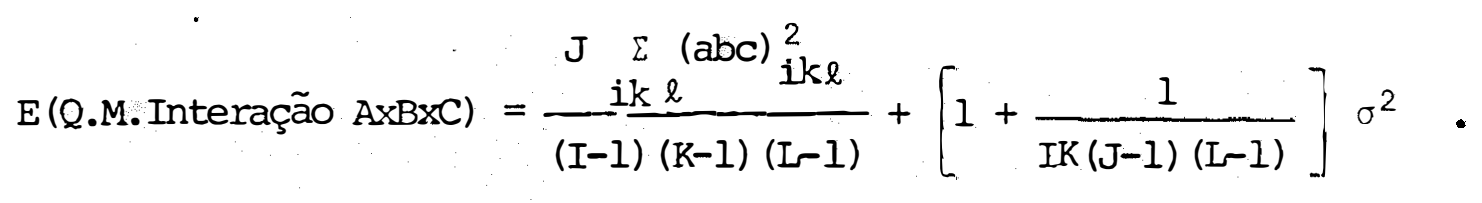

$$
\text { 4.2.14. Residuo (c) }
$$

$E[$ S.Q.Residuo (c)] = E (S.Q.Total) - E (S.Q.Subparcelas) -

$$
\text { - E[S.Q.Tratamentos (C)] - E(S.Q.Interação AxC) - }
$$$$
\text { - E(S.Q.Interação BXC) - E(S.Q.Interação } \mathrm{AxBXC})
$$

Como todas essas esperanças foram determina das anteriormente, pela substituição virá:

$$
\mathrm{E}\left[\mathrm{S} \cdot \mathrm{Q} \cdot \operatorname{Resíduo~(c)]}=[\operatorname{IK}(J-1)(L-1)-1] \sigma^{2}\right.
$$


com a expressão da esperança do quadrado médio do resíduo (c) dada por:

$$
E[Q \cdot M \cdot \operatorname{Residuo~(c)~}]=\sigma^{2}
$$

Conforme se pode observar, apenas a

$$
E[Q \cdot M \cdot R e s i d u o \quad(c)]
$$

permanece inalterada, em decorrênćia da estimação da unidade perdida, uma vez que sua contribuição para este resíduo é nula.

Em todas as expressões das esperanças dos quadrados médios obtidas, exceto a do Resíduo (c), existe, associado à variância residual $\left(\sigma^{2}\right)$, a nível de sub-subparcela, um fator

$$
g=\left[1+\frac{1}{\operatorname{IK}(J-1)(L-1)}\right] \sigma^{2} .
$$

Uma síntese do que até agora foi apresentado pode ser vista no Quadro II a seguir, onde são mostrados o esquema de análise de variância e as expressões das esperanças das somas de quadrados e dos quadrados médios, para cada componente. 
87.




88.

Verifica-se pelo Quadro II que os componentes blocos e tratamentos (A) devem ser testados com $\circ$ quadrado médio do resíduo (a) e os componentes tratamentos (B) e interação $A x B$ com o quadrado médio do resíduo (b). As de mais fontes de variação de interesse, a nível de sub-subpar cela, de uma forma prática, deverão ser testadas com o qua drado médio do resíduo (c) multiplicado do fator $g$. Esse procedimento, embora aproximado, introduz um erro máximo de $20 \%$ nos resultados do valor do teste $\mathrm{F}$ obtido, isto conside rando um caso extremo de um experimento instalado em parcelas sub-subdivididas com as seguintes especificações: 2 blo cos, 2 tratamentos primários (A), 2 tratamentos secundários (B) e 2 tratamentos terciários (C). Considerando as dimensões do exemplo proposto neste trabalho, esse erro se situa em torno de 1,3\% para os componentes testados a nível de sub-subparcela.

\subsubsection{Exemplo Ilustrativo}

Considerando os dados de "DEW-LAP" de colmos de cana-de-açúcar, foi feita, de acordo com a estrutura proposta, a análise de variância cujos resultados estão a presentados na Tabela I. 
TABELA I - Análise de Variância dos Dados de "DEW-LAP" de Colmos de Cana-de-Açúcar.

\begin{tabular}{|c|c|c|c|c|}
\hline Causas de variação & G.L. & S.Q. & Q.M. & $F$ \\
\hline Blocos & 3 & $1.421,74$ & 473,91 & \\
\hline Espaçamentos (E) & 3 & $2.027,58$ & 675,86 & $5,21 *$ \\
\hline Resíduo (a) & 9 & $1.167,95$ & 129,77 & \\
\hline Parcelas & (15) & $(4.617,27)$ & & \\
\hline Variedades (V) & 2 & $74.008,014$ & $37.004,01$ & $188,47 * *$ \\
\hline$E \times V$ & 6 & $4.765,49$ & 794,25 & $4,05 * *$ \\
\hline Residuo (b) & 24 & $4.712,06$ & 196,34 & \\
\hline Subparcelas & $(47)$ & $(88.102,83)$ & . & \\
\hline Densidades (D) & 2 & 679,51 & 339,76 & $4,11 *$ \\
\hline$E \times D$ & 6 & 834,49 & 139,08 & 1,68 \\
\hline$V \times D$ & 4 & 567,70 & 141,93 & 1,71 \\
\hline$E \times V \times D$ & 12 & $1.467,63$ & 122,30 & 1,48 \\
\hline Residuo (c) & 71 & $5.796,00$ & 81,63 & \\
\hline Total & (142) & $(97.448,16)$ & & \\
\hline
\end{tabular}

* Significativo ao limite de 5\% de probabilidade

** Significativo ao limite de l\% de probabilidade 
Para efeito de aplicação do teste F, a nível de sub-subparcela, o Q.M.Resíduo (c), foi multiplicado pelo fator $g=73 / 72$.

Como se depreende, houve diferenças significativas em todos os efeitos principais e na interação de tratamentos $E \times V$.

4.3. Estimadores de Variāncias de Contrastes de Médias Envolvendo o Tratamento com a Sub-subparcela Perdi. $\underline{\text { da }}$

No delineamento em parcelas sub-subdividi das podem ser indentificados um total de 27 casos distintos de contrastes entre médias de tratamentos principais, médias de interações duplas e médias de interações triplas. Desses contrastes foram isolados apenas 12 , os de maior in teresse, estruturados a seguir.

4.3.1. Contraste Entre Duas Médias de Tratamentos (A)

Para o tratamento $A_{1}$ onde ocorreu a perda da sub-subparcela tem-se: 


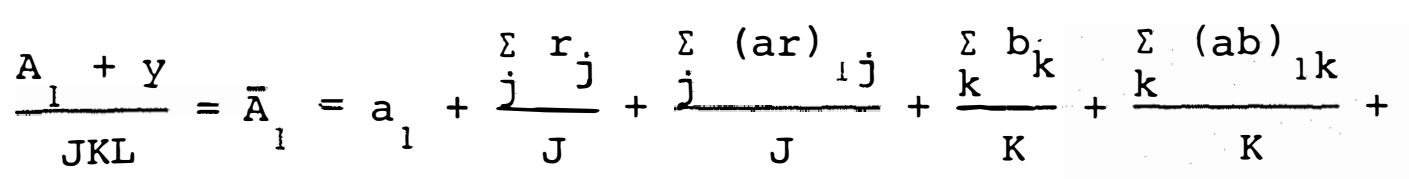

$$
\begin{aligned}
& +\frac{j k(a b r) l k j}{J K}+\frac{\ell^{\sum c_{\ell}}}{L}+\frac{\ell^{(a c)} 1 \ell}{L}+\frac{k^{\sum(b c)} k \ell}{K L}+ \\
& +\frac{\sum_{\ell}(a b c) 1 k \ell}{K L}+\frac{j k \ell e_{1 j k \ell}}{J K L}+\frac{L_{\ell=2}^{L} e_{11 I \ell}^{L}}{J K L(L-1)}+ \\
& +\frac{\sum e_{1 j k \ell}}{J K L}+\frac{\sum_{j=2}^{J} e_{1 j 11}}{J K L(J-1)}-\frac{\sum_{j=2}^{J} \sum_{\ell=2}^{L} e_{1 j 1 \ell}}{J K L(J-1)(L-1)}
\end{aligned}
$$

Em relação a qualquer outro tratamento $A_{i}$ on de não houve a perda, virá:

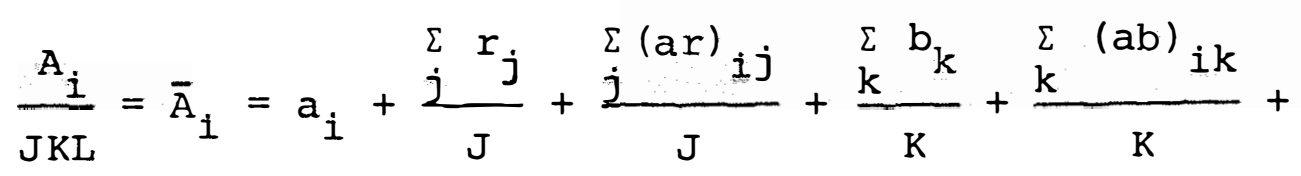

$$
\begin{aligned}
& +\frac{j k(a b r) i k j}{J K}+\frac{\sum^{\sum} c_{\ell}}{L}+\frac{\sum^{(a c)} i \ell}{I}+\frac{\sum_{\ell}^{\sum}(b c) k \ell}{K L}+ \\
& +\frac{\sum_{k \ell}(a b c) i k \ell}{K L}+\frac{j k \ell e_{i j k \ell}}{J K L}
\end{aligned}
$$




\section{Assim virá:}

$$
\begin{aligned}
& \hat{I}_{i}=\bar{A}_{i}-\bar{A}_{1}=a_{i}-a_{1}+\frac{1}{J} \sum_{j}\left[(a r)_{i j}-(a r)_{1 j}\right]+ \\
& +\frac{1}{J K} \sum_{j k}\left[(a b r)_{i k j}-(a b r)_{1 k j}\right]+\frac{\sum k e_{i j k \ell}}{J K L}- \\
& -\frac{j k \ell e_{1 j k \ell}}{J K L}-\frac{L \sum_{\ell=2}^{L} e_{111 \ell}}{J K L(L-1)}-\frac{\sum^{j} e_{1 j k \ell}}{J K L}- \\
& -\frac{\sum_{j=2}^{J} e_{1 j 11}}{J K L(J-1)}+\frac{\sum_{j=2}^{J} \sum_{l=2}^{L} e_{1 j 1 \ell}}{J K L(J-1)(L-1)} .
\end{aligned}
$$

virá:

$$
\begin{gathered}
E\left(\hat{Y}_{i}\right)=a_{i}-a_{1}, \\
\text { Por definição } \\
V\left(\hat{Y}_{i}\right)=E\left[\hat{Y}_{i}-E\left(\hat{Y}_{i}\right)\right]^{2},
\end{gathered}
$$


portanto:

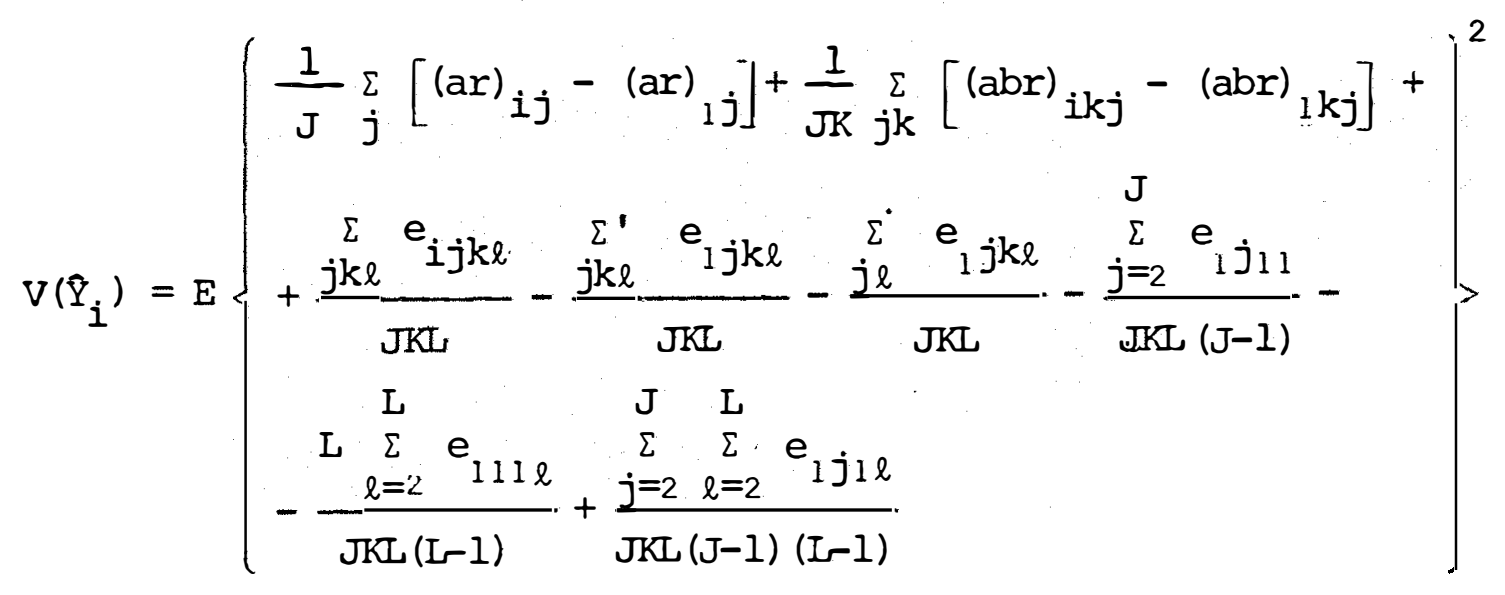

đonde se obtém:

$$
\begin{aligned}
V\left(\hat{Y}_{i}\right) & =\frac{2 J}{J^{2}} \sigma_{a r}^{2}+\frac{2 J K}{J^{2} K^{2}} \sigma_{a b r}^{2}+\frac{J K L \sigma^{2}}{J^{2} K^{2} L^{2}}+\frac{J K(L-1) \sigma^{2}}{J^{2} K^{2} L^{2}}+\frac{L(J-I) \sigma^{2}}{J^{2} K^{2} L^{2}}+ \\
& +\frac{L^{2}(I-I) \sigma^{2}}{J^{2} K^{2} L^{2}(I-1)^{2}}+\frac{(J-1) \sigma^{2}}{J^{2} K^{2} L^{2}(J-1)^{2}}+\frac{(J-1)(I-1) \sigma^{2}}{J^{2} K^{2} L^{2}(J-1)^{2}(L-1)^{2}}
\end{aligned}
$$

ou ainda:

$$
V\left(\hat{Y}_{i}\right)=\frac{2}{J K L}\left[K L \sigma_{a r}^{2}+L \sigma_{a b r}^{2}+\sigma^{2}+\frac{I \sigma^{2}}{2 \operatorname{IK}(J-1)(I-1)}\right]
$$

que associada ao Quadro II resulta:

$$
\hat{V}\left(\hat{Y}_{i}\right)=\frac{2}{J K L}\left[\text { Q.M.Residuo (a) }+\frac{(I-2) \text { Q.M.Residuo (c) }}{2 I K(J-1)(I-1)}\right]
$$




\section{Para facilitar considerar-se-á:}

$$
\begin{aligned}
& E_{a}=E[Q \cdot M \cdot \operatorname{Residuo~(a)~}] \\
& E_{b}=E[Q \cdot M \cdot \operatorname{Residuo~(b)]~} \\
& E_{c}=E[Q \cdot M \cdot \operatorname{Residuo~(c)~}]
\end{aligned}
$$

e:

$$
f=\frac{1}{2 I K(J-I)(L-I)}
$$

Assim, tem-se:

$$
\theta\left(\hat{Y}_{i}\right)=\frac{2}{J K L}\left[E_{a}+f(I-2) E_{c}\right] .
$$

A esse estimador estão associados $n_{1}^{\prime}$ graus de liberdade, que pode ser satisfatoriamente calculado aatravés da fórmula de SATTERTHWAITE que para esse caso é:

$$
n_{1}^{\prime}=\frac{\left[E_{a}+f(I-2) E_{c}\right]^{2}}{\frac{E_{a}^{2}}{n_{a}}+\frac{f^{2}(I-2)^{2} E_{c}^{2}}{n_{c}}}
$$


onde:

$$
\begin{aligned}
& \mathrm{n}_{\mathrm{a}}=\text { número de graus de liberdade do residuo (a), e: } \\
& \mathrm{n}_{\mathrm{c}}=\text { número de graus de liberdade do resíduo (c). }
\end{aligned}
$$

4.3.2. Contraste Entre Duas Médias de Tratamentos (B)

$$
\text { A média do tratamento } B_{1} \text { onde ocorreu a }
$$
perda de sub-subparcela é dada pela expressão:

$$
\begin{aligned}
& \frac{B_{1}+y}{I J L}=\bar{B}_{1}=\frac{\sum a_{i}}{I}+\frac{\sum r_{j}}{J}+\frac{\sum(a r)_{i j}}{I J}+b_{1}+\frac{\sum_{i}(a b)}{I}+\frac{\sum_{i j}(a b r)_{i l j}}{I J}+ \\
& +\frac{\ell^{\sum c_{\ell}}}{L}+\frac{\sum(\mathrm{il})_{i \ell}}{I L}+\frac{\sum(\mathrm{bc})_{1 \ell}}{L}+\frac{\sum_{i \ell}(\mathrm{abc})_{i 1 \ell}}{I L}+\frac{\sum^{\prime} e_{i j \ell} e_{i j l}}{I J L}+ \\
& +\frac{\sum_{\ell=2}^{L} e_{111 \ell}}{L L(L-1)}+\frac{J \sum_{j=2}^{J} e_{1 j 11}}{I L(J-1)}+\frac{\sum e_{i j 1 \ell}}{L L}-\frac{\sum_{j=2}^{J} \sum_{\ell=2}^{L} e_{1 j 1 \ell}}{I J L(J-1)(L-1)}
\end{aligned}
$$

Para um tratamento $B_{k}$ qualquer:

$$
\begin{aligned}
& \frac{B_{k}}{I J L}=\bar{B}_{k}=\frac{\sum a_{i}}{I}+\frac{\sum r_{j}}{J}+\frac{\sum(a r)_{i j}}{I J}+b_{k}+\frac{\sum(a b) i k}{I}+\frac{\sum(a b r) i k j}{I J}+\frac{\sum c_{\ell}}{L}+ \\
& +\frac{\sum_{i \ell}(\mathrm{ac})}{\mathrm{Il} l}+\frac{\sum^{\sum(\mathrm{bc})} \mathrm{kl}}{\mathrm{L}}+\frac{\sum(\mathrm{abc}) i k \ell}{I L}+\frac{\sum_{i j \ell} e_{i j k \ell}}{I L}
\end{aligned}
$$


96.

Então:

$$
\begin{aligned}
& \hat{\mathrm{Y}}_{k}=\bar{B}_{k}-\bar{B}_{1}=b_{k}-b_{l}+\frac{I}{I J} \sum_{i j}\left[(a b r)_{i k j}-(a b r)_{i l j}\right]+ \\
& +\frac{\sum_{i j \ell} e_{i j k \ell}}{I J L}-\frac{\sum^{\prime} e^{\prime} e_{i j l \ell}}{I J L}-\frac{\sum_{\ell} e_{i j l \ell}}{I J L}-\frac{\sum_{\ell=2}^{L} e_{111 \ell}}{I J L(L-1)}- \\
& -\frac{J \sum_{j=2}^{J} e_{1 j 11}}{\operatorname{IJL}(J-1)}+\frac{\sum_{j=2 \ell=2}^{J} e_{1 j l \ell}}{\operatorname{IJL}(J-1)(L-1)}
\end{aligned}
$$

e:

$$
E\left(\hat{Y}_{k}\right)=b_{k}-b_{l}
$$

Por definição:

$$
V\left(\hat{Y}_{k}\right)=E\left[\hat{Y}_{k}-E\left(\hat{Y}_{k}\right)\right]^{2}
$$

portanto:

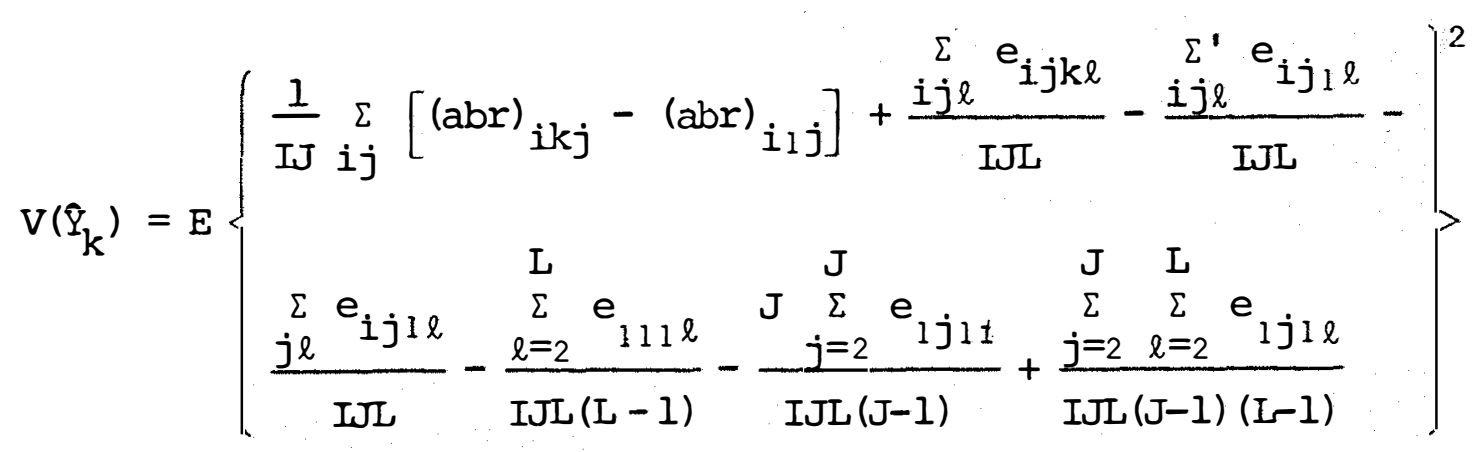




$$
\begin{aligned}
V\left(\hat{\mathrm{P}}_{\mathrm{k}}\right) & =\frac{2 I J}{I^{2} J^{2}} \sigma_{a b r}^{2}+\frac{I J L \sigma^{2}}{I^{2} J^{2} L^{2}}+\frac{J L(I-I) \sigma^{2}}{I^{2} J^{2} L^{2}}+\frac{J(L-I) \sigma^{2}}{I^{2} J^{2} L^{2}}+ \\
& +\frac{(L-I) \sigma^{2}}{I^{2} J^{2} L^{2}(L-I)^{2}}+\frac{J^{2}(J-I) \sigma^{2}}{I^{2} J^{2} L^{2}(J-I)^{2}}+\frac{(J-I)(L-I) \sigma^{2}}{I^{2} J^{2} L^{2}(J-I)^{2}(L-I)^{2}}
\end{aligned}
$$

ou ainda:

$$
V\left(\hat{Y}_{k}\right)=\frac{2}{I J L}\left[L \sigma_{a b r}^{2}+\sigma^{2}+\frac{K \sigma^{2}}{2 I K(J-I)(L-I)}\right]
$$

Seu estimador, de acordo com o Quadro II é:

$$
\hat{V}\left(\hat{Y}_{k}\right)=\frac{2}{I J L}\left[E_{b}+f(K-2) E_{c}\right]
$$

A esta variância estão associadas $n_{2}^{\prime}$ graus de liberdade que também podem ser obtidos através da fórmuça de SATTERTHWAITE, ou seja:

$$
n_{2}^{\prime}=\frac{\left[E_{b}+f(K-2) E_{c}\right]^{2}}{\frac{E_{b}^{2}}{n_{b}}+\frac{f^{2}(K-2)^{2} E_{c}^{2}}{n_{c}}}
$$

onde:

$\mathrm{n}_{\mathrm{b}}$ é o número de graus de liberdade do resíduo (b) . 
4.3.3. Contraste Entre Duas Médias de Tratamentos (C )

Para o tratamento $C_{1}$ relativo à sub-subpar cela perdida tem-se:

$$
\begin{aligned}
& \frac{c_{1}+y}{I J K}=\bar{c}_{1}=\frac{\sum a_{i}}{I}+\frac{\sum r_{j}}{J}+\frac{\sum_{j(j}(a r)_{i j}}{I J}+\frac{\sum b_{k}}{K}+\frac{\sum(a b) i k}{I K}+ \\
& +\frac{\sum_{i j k}(a b r) i k j}{I J K}+c_{1}+\frac{\sum^{i(a c)} i l}{I}+\frac{\sum_{k}(b c) k l}{K}+ \\
& +\frac{i k(a b c) i k l}{I K}+\frac{i j k}{\sum^{\prime} e_{i j k l}}+\frac{\sum^{\prime} e^{j} e_{l j k l}}{I J K}+ \\
& +\frac{\sum_{\ell=2}^{L} e_{111 \ell}}{I J K(L-1)}+\frac{J \sum_{j=2}^{J} e_{1 j 11}}{I J K(J-1)}-\frac{\sum_{j=2}^{J} \sum_{\ell=2}^{L} e_{1 j 1 \ell}}{I J K(J-1)(L-1)}
\end{aligned}
$$

Para qualquer outro tratamento $C_{l}$ fica: 


$$
\begin{aligned}
& \frac{c_{\ell}}{I J K}=\bar{c}_{\ell}=\frac{\sum a_{i}}{I}+\frac{\sum r_{j}}{J}+\frac{\sum(a r)_{i j}}{I J}+\frac{\sum b_{k}}{K}+\frac{\sum(a b) i k}{I K}+ \\
& +\frac{\sum_{j j k}(a b r)_{i k j}}{I J K}+c_{\ell}+\frac{\sum^{\sum(a c)} i \ell}{I}+\frac{\sum^{(b c)} k \ell}{K}+ \\
& +\frac{i k(a b c) i k l}{I K}+\frac{i j k}{I J K} e_{i j k \ell}
\end{aligned}
$$

Estrutura-se então:

$$
\begin{aligned}
& \hat{Y}_{\ell}=\bar{c}_{l}-\bar{c}_{1}=c_{\ell}-c_{1}+\frac{\sum e^{i j k} e_{i j k l}}{I J K}-\frac{\sum^{\prime} e_{i j k}}{I J K}-\frac{\sum_{i k} e_{1 j k l}}{I J K}- \\
& -\frac{\sum_{\ell=2}^{L} e_{111 \ell}}{I J K(L-I)}-\frac{\sum_{j=2}^{J} e_{1 j 11}}{I J K(J-I)}+\frac{\sum_{j=2}^{J} \ell_{\sum}^{L} e_{1 j 1 \ell}}{I J K(J-1)(L-1)}
\end{aligned}
$$

Como: $E\left(\hat{Q}_{\ell}\right)=c_{\ell}-c_{1}$ e $V\left(\hat{Q}_{\ell}\right)=E\left[\hat{Q}_{\ell}-E\left(\hat{Q}_{\ell}\right)\right]^{2}$ tem-se:

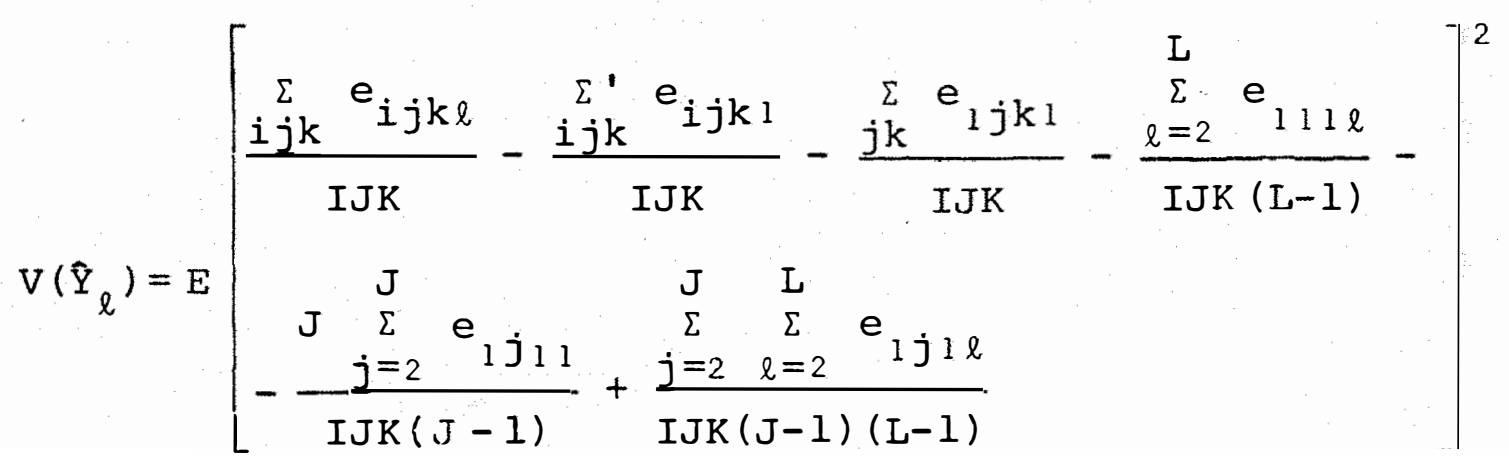


Desenvolvendo, obtēm-se:

$$
\begin{aligned}
V\left(\hat{Q}_{\ell}\right) & =\frac{I J K \sigma^{2}}{I^{2} J^{2} K^{2}}+\frac{J K(I-1) \sigma^{2}}{I^{2} J^{2} K^{2}}+\frac{J(K-1) \sigma^{2}}{I^{2} J^{2} K^{2}}+\frac{(L-1) \sigma^{2}}{I^{2} J^{2} K^{2}(L-1)^{2}}+ \\
& +\frac{J^{2}(J-1) \sigma^{2}}{I^{2} J^{2} K^{2}(J-1)^{2}}+\frac{(J-1)(L-I) \sigma^{2}}{I^{2} J^{2} K^{2}(J-1)^{2}(L-1)^{2}}
\end{aligned}
$$

ou ainda:

$$
\begin{gathered}
V\left(\hat{Y}_{\ell}\right)=\frac{2}{I J K}\left[\sigma^{2}+\frac{L \sigma^{2}}{2 I K(J-I)(L-1)}\right] \\
\text { Seu estimador é: } \\
\hat{\nabla}\left(\hat{Q}_{\ell}\right)=\frac{2 E_{C}}{I J K}[1+f . I]
\end{gathered}
$$

ao qual estão associados $\mathrm{n}_{c}$ graus de liberdade.

4.3.4. Contraste Entre Duas Médias de Tratamentos (B) Dentro do Tratamento $\left(A_{1}\right)$

Para a combinação de tratamentos $A_{1} B_{1}$ asso ciada à perda da observação tem-se: 


$$
\begin{aligned}
& \frac{A_{1} B_{1}+Y}{J L}=\frac{A_{1} B_{1}}{J}=a_{1}+\frac{\sum r_{j}}{J}+\frac{\sum(a r)_{1 j}}{J}+b_{1}+(a b)_{11}+ \\
& +\frac{\sum(a b r) 11 j}{J}+\frac{\sum^{2} c_{\ell}}{L}+\frac{\ell^{(a c)} 1 \ell}{L}+\frac{\ell^{(b c)} 1 \ell}{L}+ \\
& +\frac{\ell^{(a b c)} 11 \ell}{L}+\frac{\sum^{\prime \prime} e_{1 j 1 \ell}}{J L}+\frac{\sum^{L}=2 e_{111 \ell}}{J L(L-1)}+ \\
& +\frac{J \sum_{j=2}^{J} e_{1 j 11}}{J L(J-1)}-\frac{\sum_{j=2}^{J} \sum_{\ell=2}^{L} e_{1 j l \ell}}{J L(J-I)(L-1)}
\end{aligned}
$$

Para qualquer outra combinação de tratamentos $A_{1} B_{k}$ tem-se:

$$
\begin{aligned}
& \frac{A_{l} B_{k}}{J L}=\overline{A_{1} B_{k}}=a_{1}+\frac{\sum r_{j}}{J}+\frac{\sum(a r) 1 j}{J}+b_{k}+(a b)_{1 k}+ \\
& +\frac{\sum(a b r)_{1 k j}}{J}+\frac{\sum c_{\ell}}{L}+\frac{\ell^{(a c)} I \ell}{L}+\frac{\ell^{(b c)} k \ell}{L}+ \\
& +\frac{\sum(a b c) 1 k \ell}{L}+\frac{j \ell e_{1 j k \ell}}{J L}
\end{aligned}
$$




\section{Assim o contraste proposto}

$$
\hat{\mathrm{Y}}_{\mathrm{k} / \mathrm{i}}=\overline{\mathrm{A}_{1} \mathrm{~B}_{\mathrm{k}}}-\overline{\mathrm{A}_{1} \mathrm{~B}_{1}}
$$

\section{fica:}

$$
\begin{aligned}
& \hat{\mathrm{Y}}_{k / i}=b_{k}-b_{1}+(a b)_{1 k}-(a b)_{11}+\frac{1}{J} \sum_{j}\left[(a b r)_{1 k j}-(a b r)_{11 j}\right]+ \\
& \begin{array}{c}
+\frac{\sum e_{1 j k \ell}}{J L}-\frac{\sum l e_{1 j 1 \ell}}{J L}-\frac{\sum_{=2}^{L} e_{111 \ell}}{J L(L-1)}-\frac{J \sum_{j=2}^{J} e_{1 j 11}}{J L(J-1)}+ \\
+\frac{\sum_{j=2 \quad \sum=2 e_{1 j 1 \ell}}^{J L(J-1)(L-1)}}{J L(L)}
\end{array}
\end{aligned}
$$

$$
E\left(\underline{Q}_{k / i}\right)=b_{k}-b_{1}+(a b)_{1 k}-(a b)_{11}
$$

e

$$
V\left(\hat{Q}_{k / i}\right)=E\left[\hat{Y}_{k / i}-E\left(\hat{Q}_{k / i}\right)\right]^{2}
$$

virá: 


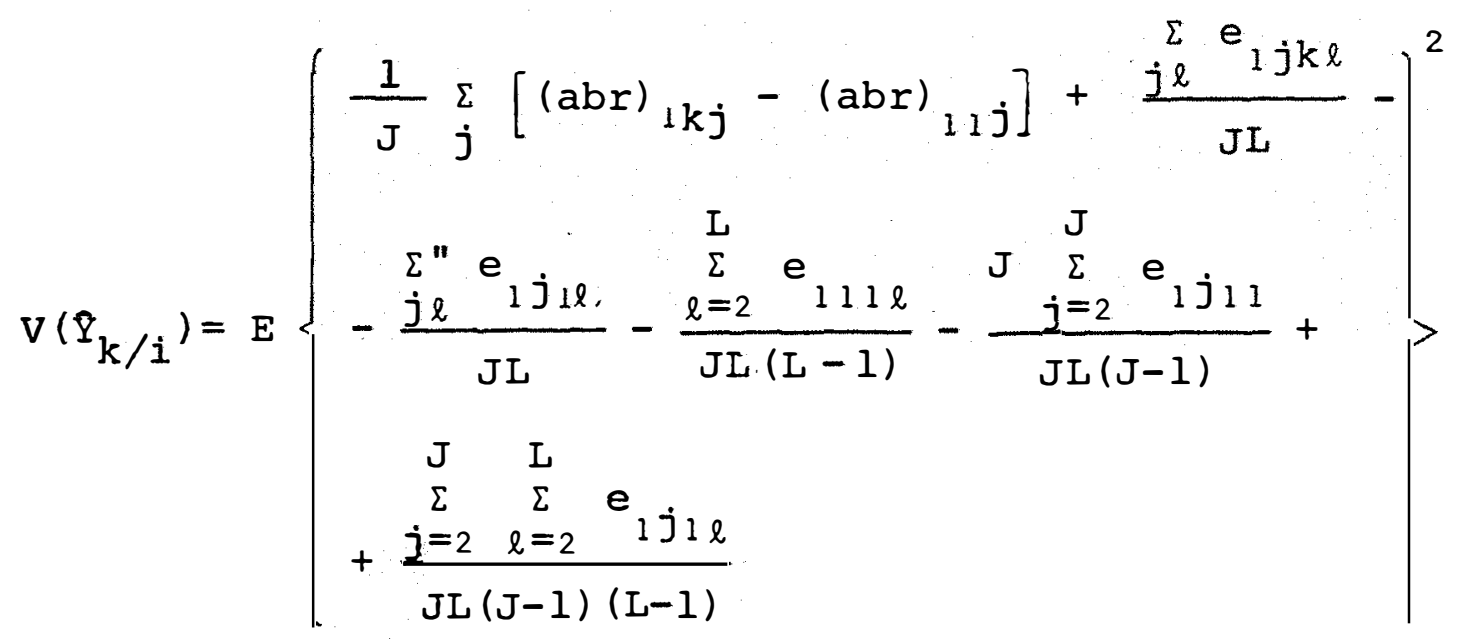

Desenvolvendo convenientemente virá:

$$
\begin{aligned}
V\left(Q_{k / i}\right) & =\frac{2 J}{J^{2}} \sigma_{a b r}^{2}+\frac{J L \sigma^{2}}{J^{2} L^{2}}+\frac{J(L-I) \sigma^{2}}{J^{2} L^{2}}+\frac{J^{2}(J-I) \sigma^{2}}{J^{2} L^{2}(J-I)^{2}}+ \\
& +\frac{(L-1) \sigma^{2}}{J^{2} L^{2}(L-I)^{2}}+\frac{(J-I)(L-I) \sigma^{2}}{J^{2} L^{2}(J-I)^{2}(L-I)^{2}}
\end{aligned}
$$

e assim,

$$
V\left(\hat{Y}_{k / i}\right)=\frac{2}{J L}\left[L \sigma_{a b r}^{2}+\sigma^{2}+\frac{I K \sigma^{2}}{2 I K(J-1)(L-1)}\right]
$$

Consequentemente, seu estimador será:

$$
\theta\left(\hat{Y}_{k / i}\right)-\frac{2}{J L}\left[E_{b}+f \cdot(I K-2) \cdot E_{c}\right]
$$


Tambēm por SATTERTHVATTE chega-se à :

$$
n_{3}^{\prime}=\frac{\left[E_{b}+f(I K-2) E_{c}\right]^{2}}{\frac{E_{b}^{2}}{n_{b}}+\frac{f^{2}(I K-2)^{2} E_{C}^{2}}{n_{c}}}
$$

graus de liberdade associados à variância anterior.

\subsubsection{Contraste Entre Duas Médias de Tratamentos}

(A) Dentro do Tratamento $\left(B_{1}\right)$

Para a combinação de tratamentos $A_{1} B_{1}$ onde ocorreu a sub-subparcela perdida tem-se, analogamente à $4.3 .4:$

$$
\begin{aligned}
& \frac{A_{1} B_{1}+y}{J L}=\overline{A_{1} B_{1}}=a_{1}+\frac{\sum r_{j}}{J}+\frac{\sum(a r)_{1 j}}{J}+b_{1}+(a b)_{11}+ \\
& +\frac{j(a b r) 11 j}{J}+\frac{\sum c_{\ell}}{L}+\frac{\sum(a c) 1 \ell}{L}+\frac{\ell^{(b c)} 1 \ell}{L}+ \\
& +\frac{\sum(a b c) 11 \ell}{L}+\frac{\sum \ell^{\prime \prime} e 1 j 1 \ell}{J L}+\frac{\sum^{L}=2 e_{111 \ell}}{J L(L-1)}+ \\
& +\frac{J \sum_{j=2}^{J} e_{1 j 11}}{J L(J-1)}-\frac{\sum_{j=2}^{J} \sum_{\ell=2}^{L} e_{1 j 1 \ell}}{J L(J-1)(L-1)}
\end{aligned}
$$




$$
\text { Para outra combinação de tratamento } A_{i}{ }^{B}
$$

qualquer, o modelo fica:

$$
\begin{aligned}
\frac{A_{i} B_{1}}{J L}=\overline{A_{i} B_{1}}= & a_{i}+\frac{\sum r_{j}}{J}+\frac{j(a r)_{i j}}{J}+b_{1}+(a b)_{i l}+\frac{\sum(a b r)_{i l j}}{J}+ \\
& +\frac{\sum c_{\ell}}{L}+\frac{\sum(a c)_{i l}+\frac{l(b c)_{1 \ell}}{L}+\frac{\sum(a b c)_{i l \ell}}{L}+}{L} \\
& +\frac{\sum l e_{i j 1 \ell}}{J L}
\end{aligned}
$$

e,

$$
\hat{\mathrm{Y}}_{i / k}=\overline{\mathrm{A}_{i} \mathrm{~B}_{1}}-\overline{\mathrm{A}_{1} \mathrm{~B}_{1}}=a_{i}-a_{1}+\frac{1}{J} \sum_{j}\left[(\operatorname{ar})_{i j}-(a r)_{1 j}\right]+(a b)_{i 1}-
$$

$$
\begin{aligned}
& -(a b)_{11}+\frac{1}{J} \sum_{j}\left[(a b r)_{i 1 j}-(a b r)_{11 j}\right]+ \\
& +\frac{\sum_{i \ell} e_{i j l \ell}}{J L}-\frac{j \ell e_{1 j l \ell}}{J L}-\frac{\sum_{\ell=2} e_{111 \ell}}{J L(L-1)}- \\
& -\frac{J \sum_{j=2} e_{1 j 11}}{J L(J-1)}+\frac{\sum_{j=2} \sum_{\ell=2}^{L} e_{1 j 1 \ell}}{J L(J-1)(L-1)}
\end{aligned}
$$




$$
E\left(\hat{Y}_{i / k}\right)=a_{i}-a_{1}+(a b)_{i 1}-(a b)_{11}
$$

e a

$$
V\left(\hat{Y}_{i / k}\right)=E\left[\hat{Y}_{i / k}-E\left(\hat{Y}_{i / k}\right)\right]^{2},
$$

substituindo tem-se:

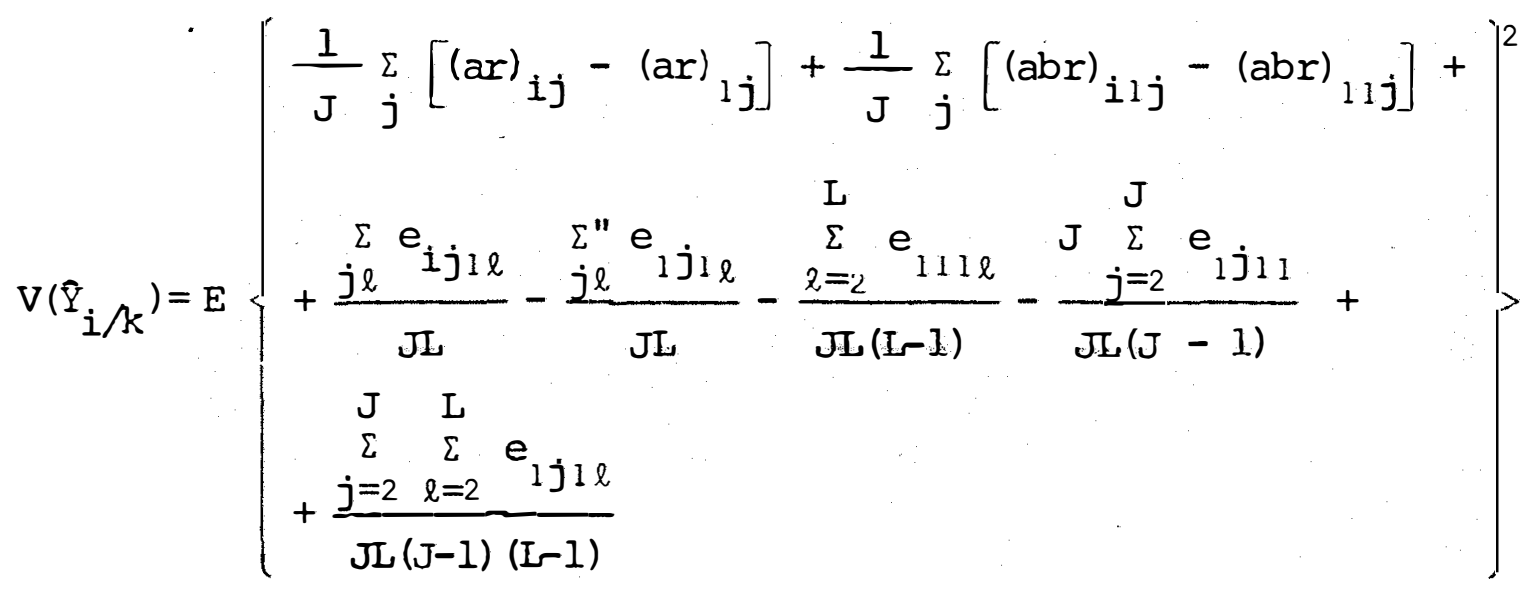

Desenvolvendo o quadrado e aplicando a espe rança virá:

$$
\begin{aligned}
V\left(\hat{Y}_{i / k}\right) & =\frac{2 J}{J^{2}} \sigma_{a r}^{2}+\frac{2 J}{J^{2}} \sigma_{a b r}^{2}+\frac{J L \sigma^{2}}{J^{2} L^{2}}+\frac{J(L-I) \sigma^{2}}{J^{2} L^{2}}+\frac{(L-I) \sigma^{2}}{J^{2} L^{2}(L-1)^{2}}+ \\
& +\frac{J^{2}(J-1) \sigma^{2}}{J^{2} L^{2}(J-I)^{2}}+\frac{(J-I)(L-1) \sigma^{2}}{J^{2} L^{2}(J-I)^{2}(L-I)^{2}}
\end{aligned}
$$


ou ainda:

$$
V\left(\hat{Y}_{i / k}\right)=\frac{2}{J K L}\left[\begin{array}{l}
K L \sigma_{a r}^{2}+L(K-1) \sigma_{a b r}^{2}+L \sigma_{a b r}^{2}+(K-1) \sigma^{2}+ \\
+\sigma^{2}+\frac{\left(I K^{2}-2\right) \sigma^{2}}{2 I K(J-1)(L-1)}+\frac{\sigma^{2}}{I K(J-I)(L-I)}
\end{array}\right]
$$

cujo estimador é dado por:

$$
\hat{V}\left(\hat{Y}_{i / k}\right)=\frac{2}{J K L}\left[E_{a}+(K-1) E_{b}^{\prime}+f\left(I K^{2}-2 K\right) E_{c}\right] .
$$

A essa variância estão associados $n_{4}^{\prime}$ graus de liberdade dado por:

$$
n_{4}^{\prime}=\frac{\left[E_{a}+(K-1) E_{b}+f\left(I K^{2}-2 K\right) E_{c}\right]^{2}}{\frac{E_{a}^{2}}{n_{a}}+\frac{(K-1)^{2} E_{b}^{2}}{n_{b}}+\frac{f^{2}\left(I K^{2}-2 K\right)^{2} E_{c}^{2}}{n_{c}}}
$$

determinado através da fórmula de SATTERTHWAITE.

4.3.6. Contraste Entre Duas Médias de Tratamentos (C) Dentro do Tratamento $\left(A_{1}\right)$

A expressão do modelo matemático representą tivo da média da combinação dos tratamentos $A_{1} C_{1}$, relativa à perda da sub-sub-unidade, é dada por: 


$$
\begin{aligned}
& \frac{A_{1} C_{1}+Y}{J K}=\overline{A_{1} C_{1}}=a_{1}+\frac{\sum r_{j}}{J}+\frac{\sum(a r)_{1 j}}{J}+\frac{\sum b_{k}}{K}+\frac{\sum(a b)_{1 k}}{K}+ \\
& +\frac{\sum_{j k}(a b r)_{1 k j}}{J k}+c_{1}+(a c)_{11}+\frac{\sum_{k}(b c)_{k l}}{k}+ \\
& +\frac{\sum^{\sum}(a b c) l k l}{K}+\frac{\sum^{\prime} e^{\prime} e_{1 j k l}}{J K}+\frac{\sum^{L}=2 e_{111 \ell}^{L}}{J K(L-1)}+ \\
& +\frac{J \sum_{j=2}^{J} e_{1 j 11}}{J K(J-1)}-\frac{\sum_{j=2}^{J} \sum_{\ell=2}^{L} e_{1 j} j_{1 \ell}}{J K(J-1)(L-1)}
\end{aligned}
$$

Para outra combinação $A_{1} C_{\ell}$ qualquer, a expressão será:

$$
\begin{aligned}
& \frac{A_{1} C_{l}}{J K}=\overline{A_{1} C_{l}}=a_{1}+\frac{\sum r_{j}}{J}+\frac{\sum(a r)_{1 j}}{J}+\frac{\sum b_{k}}{K}+\frac{\sum(a b)_{1 k}}{K}+\frac{\sum k(a b r)_{1 k j}}{J K}+ \\
& +c_{\ell}+(a c)_{1 \ell}+\frac{k^{\sum(b c)_{k \ell}}}{k}+\frac{k^{\sum(a b c)_{1 k \ell}}}{k}+ \\
& +\frac{\sum k e_{1 j k \ell}}{J K}
\end{aligned}
$$




$$
\begin{aligned}
& \text { O contraste } \hat{\mathrm{Y}}_{\ell / i}=\overline{\mathrm{A}_{1} \mathrm{C}_{\ell}}-\overline{\mathrm{A}_{1} \mathrm{C}_{1}} \text { fica: } \\
& \hat{\mathrm{Y}}_{\ell / i}=c_{\ell}-c_{1}+(a c)_{1 \ell}-(a c)_{11}+\frac{\sum_{j k} e_{1 j k \ell}}{J K}- \\
& -\frac{\sum^{\prime} e_{1 j k 1}}{J K}-\frac{\sum_{\ell=2}^{L} e_{111 \ell}}{J K(L-1)}-\frac{\sum_{j=2}^{J} e_{1 j 11}}{J K(J-1)}+ \\
& +\frac{\sum_{j=2}^{J} \ell_{=2}^{L} e_{1 j 1 \ell}}{J K(J-1)(L-1)}
\end{aligned}
$$

Como:

$$
E\left(\hat{Y}_{\ell / i}\right)=c_{\ell}-c_{1}+(a c)_{1 \ell}-(a c)_{11}
$$

e

$$
V\left(\hat{Y}_{\ell / i}\right)=E\left[\hat{Y}_{\ell / i}-E\left(\hat{Y}_{\ell / i}\right)\right]^{2}
$$

então:

$$
V\left(\hat{Y}_{\ell / i}\right)=E\left[\begin{array}{c}
\frac{j e_{1 j k \ell}}{J K}-\frac{j k e_{1 j k 1}}{J K}-\frac{\sum_{\ell=2}^{L} e_{111 \ell}}{J K(L-1)}-\frac{J \sum_{j=2}^{J} e_{1 j 11}}{J K(J-1)} \\
\begin{array}{l}
J \quad L \\
+\frac{j=2}{J K} e_{\ell=2} e_{1 j 1 \ell} \\
J K(J-1)(L-1)
\end{array}
\end{array}\right]^{2}
$$


Desenvolvendo virá:

$$
\begin{aligned}
V\left(\hat{\mathbf{Y}}_{\ell / i}\right)=\frac{J K \sigma^{2}}{J^{2} K^{2}} & +\frac{J(K-I) \sigma^{2}}{J^{2} K^{2}}+\frac{(L-1) \sigma^{2}}{J^{2} K^{2}(L-1)^{2}}+\frac{J^{2}(J-1) \sigma^{2}}{J^{2} K^{2}(J-1)^{2}}+ \\
& +\frac{(J-I)(L-1) \sigma^{2}}{J^{2} K^{2}(J-1)^{2}(L-I)^{2}}
\end{aligned}
$$

ou ainda:

$$
V\left(\hat{Y}_{\ell / i}\right)=\frac{2}{J K}\left[\sigma^{2}+\frac{I L \sigma^{2}}{2 I K(J-1)}(L-1)\right]
$$

com o estimador dado por:

$$
\theta\left(\hat{Y}_{\ell / i}\right)=\frac{2 E_{C}}{J K}[I+f . I L]
$$

e com $\mathrm{n}_{c}$ graus de liberdade associados.

4.3.7. Contraste Entre Duas Médias de Tratamentos

(A) Dentro do Tratamento $\left(C_{1}\right)$

Analogamente ao caso anterior tem-se para

a combinação $A_{1} C_{1}$ onde ocorreu a perda: 
111.

$$
\begin{aligned}
& \frac{A_{1} C_{1}+Y}{J K}=\overline{A_{1} C_{1}}=a_{1}+\frac{\sum r_{j}}{J}+\frac{\sum(a r)_{1 j}}{J}+\frac{\sum_{k} b_{k}}{K}+\frac{\sum(a b)_{1 k}}{K}+ \\
& +\frac{\sum_{k}(a b r)_{1 k j}}{J k}+c_{1}+(a c)_{11}+\frac{\sum^{\sum(b c) k l}}{k}+ \\
& +\frac{\sum(a b c) 1 k l}{K}+\frac{\sum^{\prime} e_{1 j k l}}{J K}+\frac{\sum_{\ell=2}^{L} e_{111 \ell}}{J K(L-1)}+ \\
& +\frac{J_{j=2}^{J} e_{1 j 11}}{J K(J-1)}-\frac{\sum_{j=2}^{J} \sum_{l=2}^{L} e_{1 j 1 \ell}}{J K(J-1)(L-1)}
\end{aligned}
$$

Para outra média da combinação $A_{i} C_{1}$ qualquer, tem-se:

$$
\begin{aligned}
\frac{A_{i} C_{1}}{J K}=\overline{A_{i} C_{1}} & =a_{i}+\frac{\sum r_{j}}{J}+\frac{j(a r)_{i j}}{j}+\frac{\sum_{k} b_{k}}{k}+\frac{\sum(a b)_{i k}}{k}+ \\
& +\frac{j k(a b r)_{i k j}}{J k}+c_{1}+(a c)_{i 1}+\frac{\sum(b c)_{k l}}{k}+ \\
& +\frac{\sum(a b c)_{i k 1}}{k}+\frac{\sum k e_{i j k l}}{k}
\end{aligned}
$$


112.

$$
\begin{aligned}
& \text { Assim, } 0 \text { contraste } \hat{Q}_{i / \ell}=\overline{A_{i} C_{1}}-\overline{A_{1} C_{1}} \text { fica: } \\
& q_{i / l}=a_{i}-a_{1}+\frac{1}{J} \sum_{j}\left[(a r)_{i j}-(a r)_{1 j}\right]+\frac{1}{J K} \sum_{j k}\left[(a b r)_{i k j}-(a b r)_{1 k j}\right]+ \\
& +(a c)_{i 1}-(a c)_{11}+\frac{\sum_{j k} e_{1 j k_{1}}}{J K}-\frac{\sum^{\prime} e_{1 j k 1} \sum_{\ell=2}^{L} e_{111 \ell}}{J K}- \\
& -\frac{J \sum_{j=2}^{J} e_{1 j 11}}{J K(J-1)}+\frac{\sum_{j=2}^{J} \sum_{l=2}^{L} e_{1 j 1 \ell}}{J K(J-1)(I-1)}
\end{aligned}
$$

Conhecendo a

$$
E\left(\hat{q}_{i / l}\right)=a_{i}-a_{1}+(a c)_{i 1}-(a c)_{11}
$$

e

$$
V\left(\hat{Y}_{i / \ell}\right)=E\left[\hat{Y}_{i / \ell}-E\left(\hat{Y}_{i / \ell}\right)\right]^{2}
$$

tem-se:

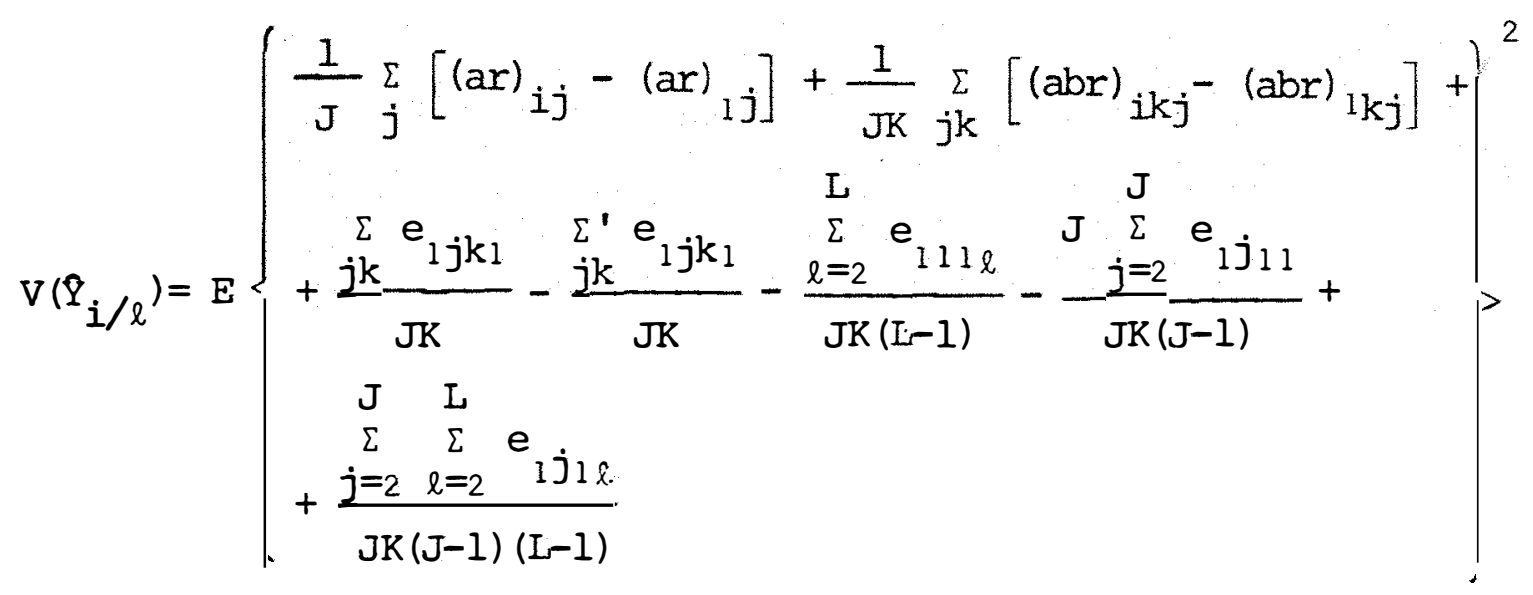


Desenvolvendo virá:

$$
\begin{aligned}
\mathrm{V}\left(\hat{\mathrm{Y}}_{\mathrm{i} / \ell}\right)=\frac{2 \mathrm{~J} \sigma_{\mathrm{ar}}^{2}}{\mathrm{~J}^{2}} & +\frac{2 \mathrm{JK} \sigma_{a b r}^{2}}{\mathrm{~J}^{2} \mathrm{~K}^{2}}+\frac{\mathrm{JK \sigma}^{2}}{\mathrm{~J}^{2} \mathrm{~K}^{2}}+\frac{\mathrm{J}(\mathrm{K}-1) \sigma^{2}}{\mathrm{~J}^{2} \mathrm{~K}^{2}}+\frac{(\mathrm{L}-1) \sigma^{2}}{\mathrm{~J}^{2} \mathrm{~K}^{2}(\mathrm{~L}-1)^{2}}+\frac{\mathrm{J}^{2}(\mathrm{~J}-1) \sigma^{2}}{\mathrm{~J}^{2} \mathrm{~K}^{2}(\mathrm{~J}-1)^{2}}+ \\
& +\frac{(\mathrm{J}-1)(\mathrm{I}-1) \sigma^{2}}{\mathrm{~J}^{2} \mathrm{~K}^{2}(\mathrm{~J}-1)^{2}(\mathrm{I}-1)^{2}}
\end{aligned}
$$

ou ainda:

$$
\begin{aligned}
& V\left(\hat{Y}_{i / \ell}\right)=\frac{2}{J K L}\left[K L \sigma_{a r}^{2}+L \sigma_{a b r}^{2}+L \sigma^{2}+\frac{I L_{\sigma}^{2} \sigma^{2}}{2 I K(J-1)(L-1)}\right] \\
& \text { O estimador dessa variância é: } \\
& \hat{V}\left(\hat{Y}_{i / \ell}\right)=\frac{2 E_{a}}{J K L}+\frac{2 E_{C}}{J K L}\left[(L-1)+f\left(I L^{2}-2\right)\right] \\
& \text { Pela fórmula de SATTERTHẈATE tem-se: } \\
& n_{5}^{\prime}=\frac{\left\{E_{a}+\left[(L-1)+f\left(I L^{2}-2\right)\right] E_{c}\right\}^{2}}{\frac{E_{a}^{2}}{n_{a}}+\frac{\left[(L-1)+f\left(I L^{2}-2\right)\right]^{2} E_{c}^{2}}{n_{c}}}
\end{aligned}
$$

que são os graus de liberdade associados à variância anterior. 
4.3.8. Contraste Entre Duas Médias de Tratamentos (C) Dentro do Tratamento $\left(B_{1}\right)$

Para a combinação de tratamentos $B_{1} C_{1}$ onde ocorreu a perda tem-se:

$$
\begin{aligned}
& \frac{B_{1} C_{1}+y}{I J}=\overline{B_{1} C_{1}}=\frac{\sum a_{i}}{I}+\frac{\sum r_{j}}{J}+\frac{\sum(a r)_{i j}}{I J}+b_{1}+\frac{\sum(a b)_{i 1}}{I}+ \\
& +\frac{\sum_{i j(a b r)} 11 j}{I J}+c_{1}+\frac{\sum(a c)_{i 1}}{I}+(b c)_{11}+ \\
& +\frac{\sum(a b c) i 11}{I}+\frac{\sum^{\prime} e_{i j 11}}{I J}+\frac{\sum^{i} e^{I} e_{111 \ell}}{I J(L-1)}+ \\
& +\frac{\sum_{j=2}^{J} e_{1 j n}}{I J(J-1)}-\frac{\sum_{j=2}^{J} \sum_{l=2}^{L} e_{1 j} j_{1 \ell}}{I J(J-1)(L-1)}
\end{aligned}
$$

Para qualquer outra combinação de tratamentos $B_{1} C_{\ell}$ onde não ocorreu a perda, virá: 


$$
\begin{aligned}
\frac{B_{1} C_{\ell}}{I J}=\frac{\sum a_{i}}{B_{1} C_{l}} & =\frac{i r_{j}}{I}+\frac{\sum(a r)_{i j}}{J}+\frac{i j b_{1}+\frac{\sum(a b)_{i l}}{I J}+}{I} \\
& +\frac{\sum(a b r)_{i l j}}{I J}+c_{\ell}+\frac{i(a c)_{i \ell}}{I}+(b c)_{1 \ell}+ \\
& +\frac{\sum(a b c)_{i l \ell}}{I}+\frac{\sum_{i j} e_{i j l \ell}}{I J}
\end{aligned}
$$

Assim, estrutura-se:

$$
\hat{\mathrm{Y}}_{\ell / \mathrm{k}}=\overline{\mathrm{B}_{1} \mathrm{C}_{\ell}}-\overline{\mathrm{B}_{1} \mathrm{C}_{1}}
$$

ou ainda:

$$
\begin{aligned}
& \hat{\mathrm{Y}}_{\ell / \mathrm{k}}=c_{\ell}-c_{1}+(b c)_{1 \ell}-(b c)_{11}+\frac{\sum_{i j} e_{i j 1 \ell}}{I J}- \\
& -\frac{\sum_{i j}^{\prime} e_{i j 11}}{I J}-\frac{\sum_{\ell=2}^{L} e_{111 \ell}}{I J(L-1)}-\frac{J \sum_{j=2}^{J} e_{1 j 11}}{I J(J-1)}+ \\
& +\frac{\sum_{j=2}^{J} \quad \ell_{=2}^{L} \quad e_{1 j l \ell}}{I J(J-1)(L-1)}
\end{aligned}
$$

Sabendo que:

$$
E\left(\hat{Y}_{\ell / k}\right)=c_{\ell}-c_{1}+(b c)_{1 \ell}-(b c)_{11}
$$


e ainda:

$$
V\left(\hat{Y}_{\ell / k}\right)=E\left[\hat{Y}_{\ell / k}-E\left(\hat{Y}_{\ell / k}\right)\right]^{2}
$$

então:

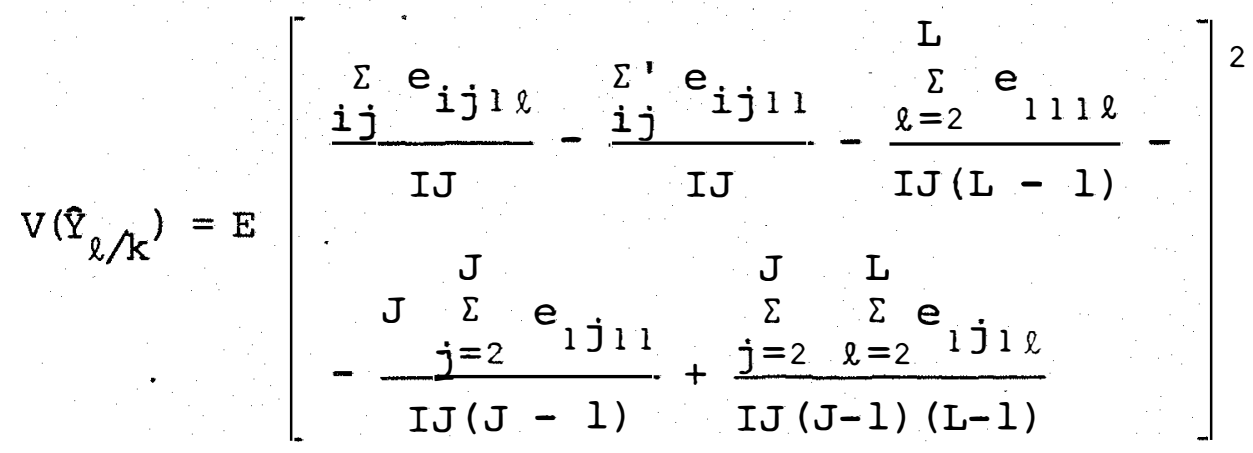

Desenvolvendo virá:

$$
\begin{aligned}
V\left(\hat{Y}_{\ell / k}\right) & =\frac{I J \sigma^{2}}{I^{2} J^{2}}+\frac{J(I-I) \sigma^{2}}{I^{2} J^{2}}+\frac{(L-I) \sigma^{2}}{I^{2} J^{2}(L-I)^{2}}+ \\
& +\frac{J^{2}(J-I)}{I^{2} J^{2}(J-1)^{2}}+\frac{\sigma^{2}}{I^{2} J^{2}(J-I)^{2}(L-I)^{2}}
\end{aligned}
$$

Portanto:

$$
V\left(\hat{Q}_{\ell / k}\right)=\frac{2}{I J}\left[\sigma^{2}+\frac{K L \sigma^{2}}{2 I K(J-1)(L-1)}\right]
$$


cujo estimador é dado por:

$$
\hat{\nabla}\left(\hat{Y}_{\ell / K}\right)=\frac{2 E_{C}}{I J}[1+f \cdot K L]
$$

com $\mathrm{n}_{\mathrm{c}}$ graus de liberdade associados.

4.3.9. Contraste Entre Duas Médias de Tratamentos (B) Dentro do Tratamento $\left(C_{1}\right)$

A expressão do mod̉elo matemático referente à combinação dos tratamentos $B_{k} C_{\ell}$ onde ocorreu a perda, é da da por:

$$
\begin{aligned}
& \frac{B C_{1}+y}{I J}=\overline{B_{1} C_{1}}=\frac{\sum a_{i}}{I}+\frac{\sum r_{j}}{J}+\frac{\sum_{j(j}(a r)_{i j}}{I J}+b_{1}+\frac{\sum^{i}(a b)_{i 1}}{I}+ \\
& +\frac{\sum_{i j(a b r)}^{i l j}}{I J}+c_{1}+\frac{\sum_{i}(a c)_{i l}}{I}+(b c)_{11}+ \\
& +\frac{\sum_{i}(a b c)_{i 11}}{I}+\frac{\sum_{i j}^{\prime} e_{i j 11}}{I J}+\frac{\sum^{L}=2 e_{111 \ell}}{I J(L-1)}+ \\
& +\frac{J \sum_{j=2}^{J} e_{1 j 11}}{I J(J-1)}-\frac{\sum_{j=2}^{J} \sum_{\ell=2}^{L} e_{1 j 1 \ell}}{I J(J-I)(L-I)}
\end{aligned}
$$


Para qualquer outra combinação de tratamentos $B_{k} C_{1}$ tem-se:

$$
\begin{aligned}
& \frac{B_{k} C_{1}}{I J}=\overline{B_{k} C_{1}}=\frac{\sum a_{i}}{I}+\frac{\sum r_{j}}{J}+\frac{\sum(a r) i j}{I J}+b_{k}+\frac{\sum(a b) i k}{I}+ \\
& +\frac{\sum_{i j}(a b r) i k j}{I J}+c_{1}+\frac{\sum^{\sum(a c)_{i l}}}{I}+(b c)_{k l}+ \\
& +\frac{\sum^{i(a b c)_{i k l}}}{I}+\frac{\sum_{i j} e_{i j k l}}{I J} \\
& \text { O contraste de interesse } \\
& \hat{\mathrm{Y}}_{\mathrm{k} / \ell}=\overline{\mathrm{B}_{\mathrm{k}} \mathrm{C}_{1}}-\overline{\mathrm{B}_{1} \mathrm{C}_{1}},
\end{aligned}
$$

expresso atravēs do modelo, fica:

$$
\begin{aligned}
\hat{P}_{k / \ell} & =b_{k}-b_{1}+\frac{I}{I J} \sum_{i j}\left[(a b r)_{i k j}-(a b r)_{i l j}\right]+(b c)_{k l}- \\
& -(b c)_{11}+\frac{\sum_{i j} e_{i j k 1}}{I J}-\frac{\sum_{i j}^{\prime} e_{i j 11}}{I J}-\frac{\sum_{\ell=2} e_{111 \ell}}{I J(L-1)}- \\
& -\frac{J \sum_{j=2} e_{1 j 11}}{I J(J-1)}+\frac{\sum_{j=2} \sum_{\ell=2} e_{1 j 1 \ell}}{I J(J-1)(L-1)}
\end{aligned}
$$




$$
\begin{aligned}
& \text { Como, } \\
& E\left(\hat{\mathrm{Y}}_{\mathrm{k} / \ell}\right)=b_{\mathrm{k}}-\mathrm{b}_{1}+(\mathrm{bc})_{k 1}-(\mathrm{bc})_{11}
\end{aligned}
$$

e

$$
V\left(\hat{\underline{Y}}_{\mathrm{k} / \ell}\right)=E\left[\hat{\mathrm{Y}}_{\mathrm{k} / \ell}-E\left(\hat{\mathrm{Y}}_{\mathrm{k} / \ell}\right)\right]^{2}
$$

então:

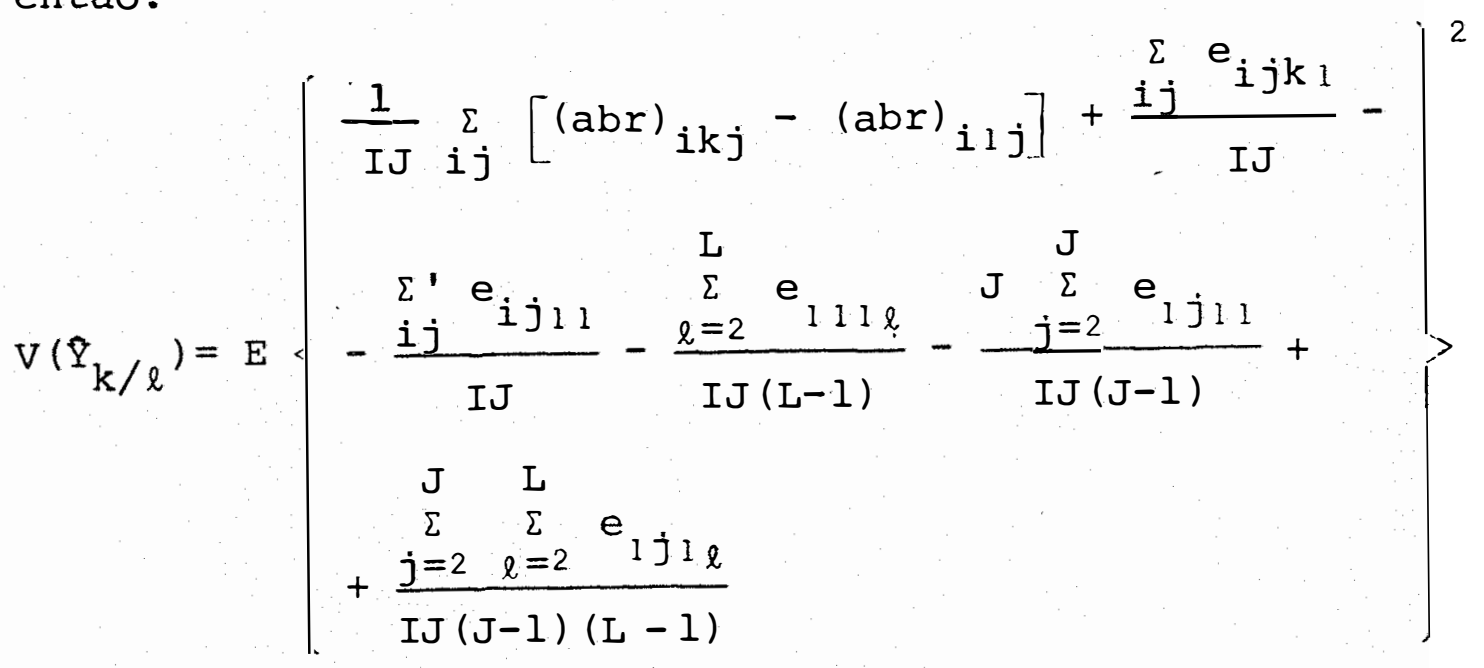

Desenvolvendo virá:

$$
\begin{aligned}
V\left(\hat{Y}_{k / \ell}\right) & =\frac{2 I J \sigma_{a b r}^{2}}{I^{2} J^{2}}+\frac{I J \sigma^{2}}{I^{2} J^{2}}+\frac{J(I-I) \sigma^{2}}{I^{2} J^{2}}+\frac{(L-I) \sigma^{2}}{I^{2} J^{2}(I-I)^{2}}+ \\
& +\frac{J^{2}(J-1) \sigma^{2}}{I^{2} J^{2}(J-1)^{2}}+\frac{(J-I)(L-I) \sigma^{2}}{I^{2} J^{2}(J-I)^{2}(L-I)^{2}}
\end{aligned}
$$


ou ainda:

$$
\mathrm{V}\left(\hat{\mathrm{Y}}_{\mathrm{k} / \ell}\right)=\frac{2}{\mathrm{IJL}}\left[\mathrm{L} \sigma_{\mathrm{abr}}^{2}+\mathrm{L} \sigma^{2}+\frac{\mathrm{KL}^{2} \sigma^{2}}{2 \mathrm{IK}(J-1)(\mathrm{L}-1)}\right]
$$

A expressão do estimador dessa variância é dada por:

$$
\nabla\left(\hat{\vartheta}_{k / \ell}\right)=\frac{2 E_{b}}{I J L}+\frac{2 E_{c}}{I J L}\left[(L-1)+f\left(K L^{2}-2\right)\right]
$$

Pelo mesmo processo utilizado anteriormente tem-se:

$$
n_{6}^{\prime}=\frac{\left\{E_{b}+\left[(L-1)+f\left(K L^{2}-2\right)\right] E_{c}\right\}^{2}}{\frac{E_{b}^{2}}{n_{b}}+\frac{\left[(L-1)+f\left(K L^{2}-2\right)\right]^{2} E_{c}^{2}}{n_{c}}}
$$

graus de liberdade.

\subsubsection{Contraste Entre Duas Médias de Tratamentos} (C) Dentro dos Tratamentos $\left(A_{1}\right)$ e $\left(B_{1}\right)$ :

Onde ocorreu a perda de sub-subparcela, a média da combinação dos tratamentos $A_{1} B_{1} C_{1}$, é dada por: 


$$
\begin{aligned}
\frac{A_{1} B_{1} C_{1}+y}{J}=\frac{A_{1} B_{1} C_{1}}{J} & a_{1}+\frac{\sum_{j}}{J}+\frac{\sum(a r)_{1 j}}{J}+b_{1}+(a b)_{11}+ \\
& +\frac{\sum(a b r)_{11 j}}{J}+c_{1}+(a c)_{11}+(b c)_{11}+ \\
& +(a b c)_{111}+\frac{j=2 e_{1 j 11}}{(J-1)}+\frac{\sum_{\ell=2}^{L} e_{111 \ell}}{J(L-1)}- \\
& -\frac{\sum_{J} \sum_{\sum} e_{1 j 1 \ell}}{J(J-1)(L-1)}
\end{aligned}
$$

Para qualquer outra combinação $A_{1} B_{1} C_{\ell}$ a ex pressão do modelo fica:

$$
\begin{aligned}
\frac{A_{1} B_{1} C_{l}}{J}=\overline{A_{1} B_{1} C_{\ell}}= & a_{1}+\frac{\sum r_{j}}{J}+\frac{\sum(a r)_{1 j}}{J}+b_{1}+(a b)_{11}+ \\
& +\frac{\sum(a b r)_{11 j}}{J}+c_{\ell}+(a c)_{1 \ell}+(b c)_{1 \ell}+ \\
& +(a b c)_{11 \ell}+\frac{\sum e_{1 j l \ell}}{J}
\end{aligned}
$$




$$
0 \text { contraste } \hat{Y}_{\ell / i k}=\overline{A_{1} B_{1} C_{\ell}}-\overline{A_{1} B_{1} C_{1}} \text {, em }
$$
relação ao modelo matemático, será expresso por:

$$
\begin{aligned}
\hat{\mathrm{Y}}_{\ell / i k}= & c_{\ell}-c_{1}+(a c)_{1 \ell}-(a c)_{11}+(b c)_{1 \ell}-(b c)_{11}+ \\
& +(a b c)_{11 \ell}-(a b c)_{111}+\frac{\sum_{J} e_{1 j 1 \ell}}{J}-\frac{\sum_{j=2} e_{1 j 11}}{J-1}- \\
& L \sum_{1} e_{111 \ell}+\frac{\sum_{j=2} \sum_{\ell=2} e_{1 j 1 \ell}}{J(J-1)(L-1)} \\
- & \frac{\ell=2}{J(L-1)}
\end{aligned}
$$

Mas,

$E\left(\hat{Y}_{\ell / i k}\right)=c_{\ell}-c_{1}+(a c)_{1 \ell}-(a c)_{11}+(b c)_{1 \ell}-(b c)_{11}+$

$$
+(a b c)_{11 \ell}-(a b c)_{111}
$$

e:

$$
V\left(\hat{Y}_{\ell / i k}\right)=E\left[\hat{Y}_{\ell / i k}-E\left(\hat{Y}_{\ell / i k}\right)\right]^{2}
$$

Assim, tem-se:

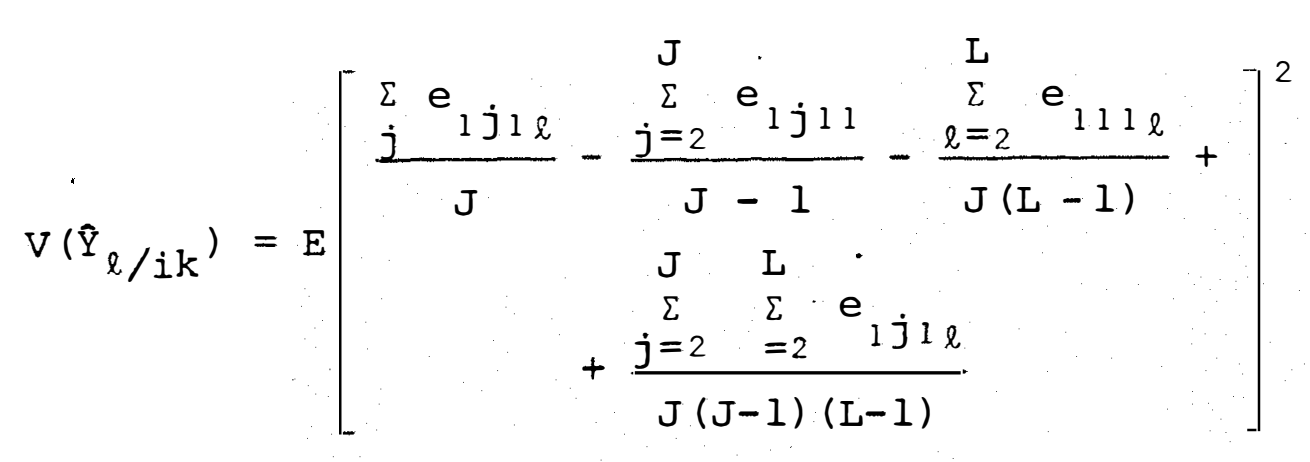


Portanto:

$V\left(\hat{Y}_{\ell / i k}\right)=\frac{J \sigma^{2}}{J^{2}}+\frac{(J-1) \sigma^{2}}{(J-1)^{2}}+\frac{(L-1) \sigma^{2}}{J^{2}(L-1)^{2}}+\frac{(J-1)(L-1) \sigma^{2}}{J^{2}(J-1)^{2}(L-1)^{2}}$

e:

$$
V\left(\hat{Y}_{\ell / i k}\right)=\frac{2}{J}\left[\sigma^{2}+\frac{I K L \sigma^{2}}{2 I K(J-1)(L-1)}\right]
$$

ou ainda:

$$
\hat{\nabla}\left(\hat{Y}_{\ell / i k}\right)=\frac{2 E_{c}}{J}[1+f . I K L]
$$

com $\mathrm{n}_{\mathrm{c}}$ graus de liberdade associados.

4.3.11. Contraste Entre Duas Médias de Tratamentos (B) Dentro dos Tratamentos $\left(A_{1}\right)$ e $\left(C_{1}\right)$

A média relativa à unidade perdida è dada por: 


$$
\begin{aligned}
& \frac{A_{1} B_{1} C_{1}+y}{J}=\overline{A_{1} B_{1} C_{1}}=a_{1}+\frac{\sum r_{j}}{J}+\frac{\sum(a r)_{1 j}}{j}+b_{1}+(a b)_{11}+ \\
& +\frac{\sum(a b r)_{11 j}}{J}+c_{1}+(a c)_{11}+(b c)_{11}+ \\
& +(a b c)_{111}+\frac{\sum_{j=2}^{J} e_{1 j 11}}{(J-1)}+\frac{\sum_{=2}^{L} e_{111 \ell}}{J(L-1)}- \\
& -\frac{\sum_{j=2}^{J} \quad \stackrel{L}{=} e_{1 j l \ell}}{J(J-1)(L-1)}
\end{aligned}
$$

Para os tratamentos correspondentes, sem a perda, a média será:

$$
\begin{aligned}
\frac{A_{1} B_{k} C_{1}}{J}=\frac{A_{1} B_{k} C_{1}}{J} & =a_{1}+\frac{j r_{j}}{J}+\frac{j(a r)^{j} j}{J}+b_{k}+(a b)_{1 k}+ \\
& +\frac{\sum(a b r)_{1 k j}}{J}+c_{1}+(a c)_{11}+(b c)_{k 1}+ \\
& +(a b c)_{1 k l}+\frac{j e_{1 j k l}}{J}
\end{aligned}
$$




\section{Como:}

$$
\begin{aligned}
& \mathrm{Y}_{k / i l}=\overline{A_{1} B_{k} C_{1}}-\overline{A_{1} B_{1} C_{1}}=b_{k}-b_{1}+(a b)_{1 k}-(a b)_{11}+ \\
& +\frac{1}{J} \sum_{j}\left[(a b r)_{1 k j}-(a b r)_{11 j}\right]+ \\
& +(b c)_{k 1}-(b c)_{11}+(a b c)_{1 k l}- \\
& -(a b c)_{111}+\frac{\sum e_{1 j k l}}{J}-\frac{j=2 e_{1 j l 1}^{J}}{J-1}- \\
& -\frac{\sum_{=2}^{L} e_{111 \ell}}{J(L-1)}+\frac{\sum_{j=2}^{J} \sum_{l=2}^{L} e_{1 j 1 \ell}}{J(J-I)(L-1)}
\end{aligned}
$$

mas:

$$
\begin{aligned}
& E\left(\hat{Y}_{k / i l}\right)=b_{k}-b_{1}+(a b)_{1 k}-(a b)_{11}+(b c)_{k 1}-(b c)_{11}+ \\
& +(a b c)_{1 k 1}-(a b c)_{111} \\
& e: \\
& V\left(Q_{k / i \ell}\right)=E\left[Q_{k / i l}-E\left(\hat{Q}_{k / i l}\right)\right]^{2}
\end{aligned}
$$


Portanto:

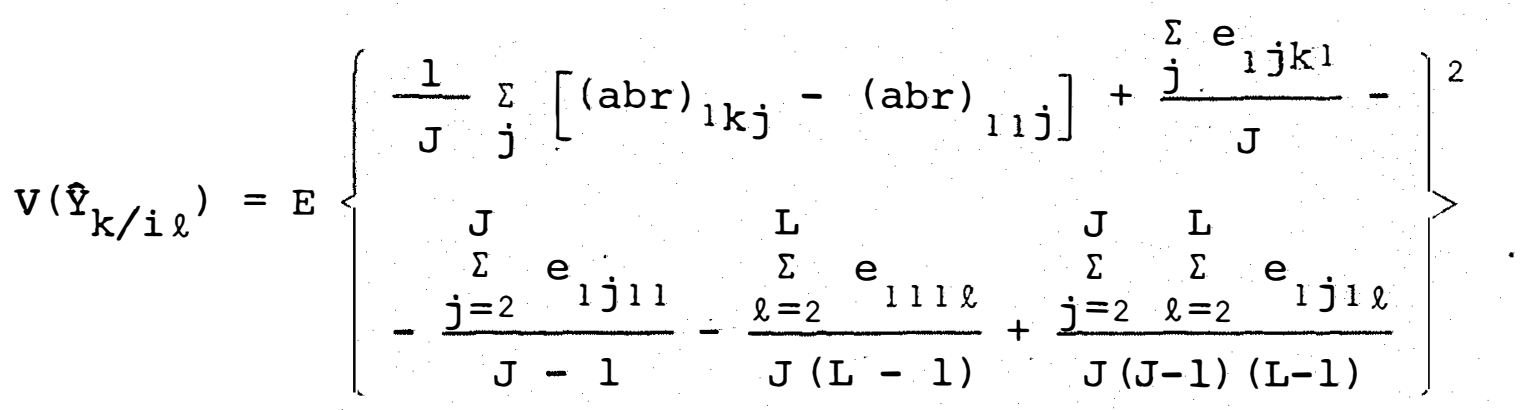

Desenvolvendo virá:

$V\left(\hat{Q}_{k / i \ell}\right)=\frac{2 J \sigma_{a b r}^{2}}{J^{2}}+\frac{J \sigma^{2}}{J^{2}}+\frac{(J-1) \sigma^{2}}{(J-1)^{2}}+\frac{(L-1) \sigma^{2}}{J^{2}(L-1)^{2}}+\frac{(J-1)(L-I) \sigma^{2}}{J^{2}(J-I)^{2}(L-1)^{2}}$

ou:

$$
V\left(\hat{\mathrm{Y}}_{\mathrm{k} / \mathrm{i} \ell}\right)=\frac{2}{\mathrm{JL}}\left[\mathrm{L} \sigma_{\mathrm{abr}}^{2}+\mathrm{L} \sigma^{2}+\frac{I K L^{2} \sigma^{2}}{2 I K(J-I)(L-I)}\right]
$$

e a expressão referente a seu estimador será:

$$
\hat{V}\left(Y_{k / i \ell}\right)=\frac{2 E_{b}}{J L}+\frac{2 E_{c}}{J L}\left[(L-1)+f\left(I K L^{2}-2\right)\right]
$$

estando associados $n_{7}^{\prime}$ graus de liberdade, dado por:

$$
n_{7}^{\prime}=\frac{\left\{E_{b}+\left[(L-1)+f\left(I K L^{2}-2\right)\right] E_{c}\right\}^{2}}{\frac{E_{b}^{2}}{n_{b}}+\frac{\left[(L-1)+f\left(I K L^{2}-2\right)\right]^{2} E_{c}^{2}}{n_{c}} .}
$$


4.3.12. Contraste Entre Duas Médias de Tratamentos (A) Dentro dos Tratamentos $\left(B_{1}\right)$ e $\left(C_{1}\right)$

Para a combinação dos tratamentos $\mathrm{A}_{1} \mathrm{~B}_{1} \mathrm{C}_{1}$ rela tiva à perda da observação a expressão do modelo é a mesma que foi apresentada nos dois casos anteriores $(4.3 .10 \mathrm{e}$ 4.3.11). Assim será apresentada apenas a relativa aos denais tratamentos correspondentes à combinação $A_{i} B_{1} C_{1}$, que è dada por:

$$
\begin{aligned}
\frac{A_{i} B_{1} C_{1}}{J}=\frac{A_{i} B_{1} C_{1}}{J} & =a_{i}+\frac{j_{j}}{J}+\frac{\sum(a r)_{i j}}{J}+b_{1}+(a b)_{i 1}+ \\
& +\frac{\sum(a b r)_{i 1 j}}{J}+c_{1}+(a c)_{i 1}+(b c)_{11}+ \\
& +(a b c)_{i 11}+\frac{\sum e_{i j 11}}{J}
\end{aligned}
$$

$$
\text { Assim, o contraste } \hat{\mathrm{Y}}_{i / k l}=\overline{\mathrm{AB}_{1} \mathrm{C}_{1}}-\overline{\mathrm{A}_{1} \mathrm{~B}_{1} \mathrm{C}_{1}} \text {, }
$$
em função do modelo matemático, será expresso por: 


$$
\begin{aligned}
\hat{Y}_{i / k \ell} & =a_{i}-a_{1}+\frac{1}{J} \sum_{j}\left[(a r)_{i j}-(a r)_{1 j}\right]+(a b)_{i 1}-(a b)_{11}+ \\
& +\frac{1}{J} \sum_{j}\left[(a b r)_{i 1 j}-(a b r)_{11 j}\right]+(a c)_{i 1}-(a c)_{11}+ \\
& +(a b c)_{i 11}-(a b c)_{111}+\frac{\sum_{j} e_{i j 11}}{J}-\frac{\sum_{j=2} e_{1 j 11}}{J-1}- \\
& -\frac{\sum_{\ell=2} e_{111 \ell}}{J(L-1)}+\frac{\sum_{j=2} \sum_{\ell=2} e_{1 j 1 \ell}}{J(J-1)(L-1)}
\end{aligned}
$$

Mas,

$$
\begin{gathered}
E\left(\hat{Y}_{i / k \ell}\right)=a_{i}-a_{1}+(a b)_{i 1}-(a b)_{11}+(a c)_{i 1}-(a c)_{11}+ \\
+(a b c)_{i 11}-(a b c)_{111}
\end{gathered}
$$

e:

$$
\mathrm{V}\left(\hat{\mathrm{Y}}_{i / k \ell}\right)=E\left[\hat{\mathrm{Y}}_{i / k \ell}-\mathrm{E}\left(\hat{\mathrm{Y}}_{i / k \ell}\right)\right]^{2}
$$


Portanto:

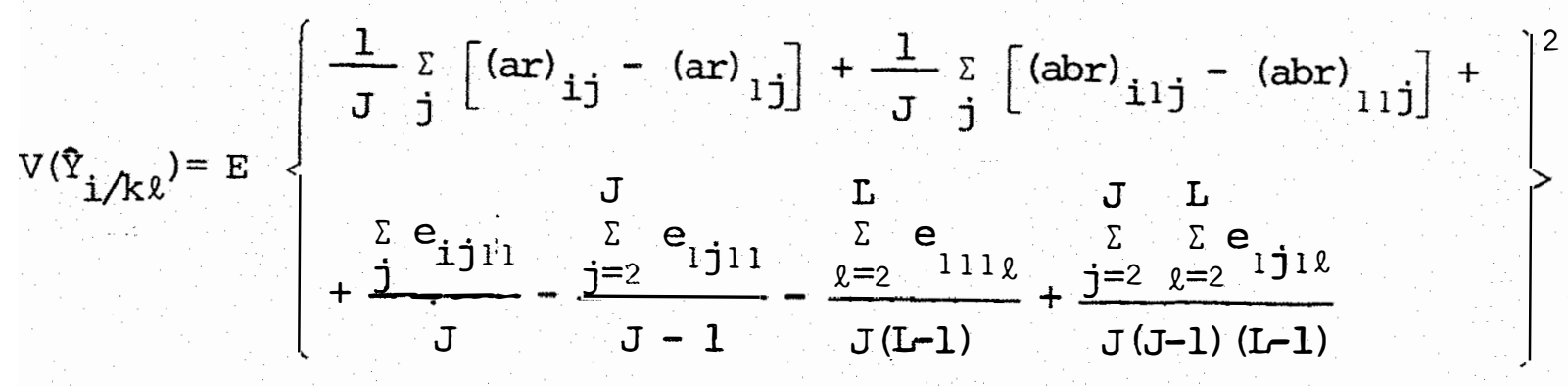

Desenvolvendo virá:

$$
\begin{aligned}
V .\left(\hat{Y}_{i / k \ell}\right) & =\frac{2 J \sigma_{a r}^{2}}{J^{2}}+\frac{2 J \sigma_{a b r}^{2}}{J^{2}}+\frac{J \sigma^{2}}{J^{2}}+\frac{(J-l) \sigma^{2}}{(J-1)^{2}}+ \\
& +\frac{(L-1) \sigma^{2}}{J^{2}(L-1)^{2}}+\frac{(J-1)(L-1) \sigma^{2}}{J^{2}(J-1)^{2}(L-1)^{2}}
\end{aligned}
$$

ou ainda:

$V\left(\hat{Y}_{i / k \ell}\right)=\frac{2}{J K L}\left[K L \sigma_{a r}^{2}+K L \sigma_{a b r}^{2}+K L \sigma^{2}+\frac{I K^{2} L^{2} \sigma^{2}}{2 I K(J-I)(L-I)}\right]$

Assim o estimador dessa variância será:

$$
\hat{V}\left(\hat{Y}_{i / k \ell}\right)=\frac{2 E_{a}}{J K L}+\frac{2(K-I) E_{b}}{J K L}+\frac{2 E_{c}}{J K L}\left[K(L-I)+f\left(I K^{2} L^{2}-2 K\right)\right]
$$


A essa variância estão associados $n_{8}^{\prime}$ graus de liberdade, que pela fórmula de SATTERTHWAITE resulta:

$$
n_{8}^{\prime}=\frac{\left.E_{a}+(K-1) E_{b}+\left[K(L-I)+f\left(I K^{2} L^{2}-2 K\right)\right] E_{c}\right\}^{2}}{\frac{E_{a}^{2}}{n_{a}}+\frac{(K-1)^{2}}{E_{b}}-\frac{\left[K(L-1)+f\left(I K^{2} L^{2}-2 K\right)\right]^{2} E_{c}^{2}}{n_{c}}+\frac{[K}{n_{b}}}
$$

\subsubsection{Exemplo Ilustrativo}

Em complementaçãotaos resultados obtidos em 4.2.15 são apresentados na Tabela II os valores das estima tivas das variâncias das estimativas de contrastes com e sem envolvimento da sub-subparcela perdida, assim como o res pectivo número de graus de liberdade associado a cada caso. Verifica-se pela Tabela II a boa concordância existente en tre as variâncias e o número de graus de liberdade para os dois casos em apreço. 
TABELA II - Resumo dos resultados das estimativas de variâncias de contrastes para os casos com e sem a perda da sub-subparcela, com os respectivos graus de liberdade associados.

\begin{tabular}{ccccc}
\hline & \multicolumn{4}{c}{ VARIÂNCIAS } \\
\cline { 2 - 5 } CONTRASTES & SEM A PERDA DA UNIDADE & COM A PERDA DA UNIDADE \\
\cline { 2 - 5 } & $0(\hat{Y})$ & $\mathrm{n}$ & $\theta(\hat{\mathrm{Y}})$ & $\mathrm{n}$ \\
\hline$\hat{\mathrm{Y}}_{\mathrm{i}}$ & 7,2094 & 9 & 7,2725 & 9 \\
$\hat{\mathrm{Y}}_{\mathrm{k}}$ & 8,1808 & 24 & 8,2045 & 24 \\
$\hat{\mathrm{Y}}_{\ell}$ & 3,4485 & 71 & 3,4721 & 71 \\
$\hat{\mathrm{Y}}_{\mathrm{k} / \mathrm{i}}$ & 32,7233 & 24 & 33,6681 & 25 \\
$\hat{\mathrm{Y}}_{\mathrm{i} / \mathrm{k}}$ & 29,0246 & 33 & 29,9698 & 35 \\
$\hat{\mathrm{Y}}_{\ell / \mathrm{i}}$ & 13,7940 & 71 & 14,7388 & 71 \\
$\hat{\mathrm{Y}}_{\mathrm{i} / \ell}$ & 16,4050 & 39 & 17,3502 & 42 \\
$\hat{\mathrm{Y}}_{\ell / k}$ & 10,3458 & 71 & 10,8415 & 71 \\
$\hat{\mathrm{Y}}_{\mathrm{k} / \ell}$ & 15,0777 & 66 & 15,5738 & 68 \\
$\hat{\mathrm{Y}}_{\ell / i k}$ & 41,3819 & 71 & 51,0188 & 71 \\
$\hat{\mathrm{Y}}_{\mathrm{k} / \mathrm{i} \ell}$ & 59,9337 & 66 & 69,9481 & 76 \\
$\hat{\mathrm{Y}}_{\mathrm{i} / \mathrm{k} \ell}$ & 56,6133 & 88 & 66,2498 & 99 \\
\hline
\end{tabular}


132 .

5. CONCLUSOES

Do presente trabalho é possivel concluir:

a) A estimativa de uma ou mais sub-subparcelas perdidas pode ser obtida, independentemente, em cada núcleo $A_{i} B_{k} \cdot$

b) o estimador $y=\frac{J\left(A_{i} B_{k} R_{j}\right)+L\left(A_{i} B_{k} C_{l}\right)-A_{i} B_{k}}{(J-I)(L-1)} \quad \bar{e}$ não tendencioso uma vez que não introduz erro adicional a - nível de sub-subparcela, que é o nível de ocorrência da per da da observação.

c) Independentemente das correções "U S", que devem ser utilizadas para as somas de quadrados, todas as expressões da esperança dos quadrados médios estão afetadas de um 
fator $g=\left[I+\frac{1}{I K(J-1)(L-1)}\right]$, associado à variância re sidual $\left(\sigma^{2}\right)$ a nível de sub-subparcela. Uma única exceção é feita à E[Q.M.Resíduo (c)] que está corretamente estimada.

d) Observa-se uma identidade nas expressões obtidas para a esperança da correção e da esperança do quadrado mé dio do resíduo (a), isto é:

$$
\mathrm{E}[\mathrm{Q} \cdot \mathrm{M} \cdot \operatorname{Residuo}(\mathrm{a})]=\mathrm{E}(\mathrm{C})=\mathrm{KL} \sigma_{\mathrm{ar}}^{2}+\mathrm{L} \sigma_{\mathrm{abr}}^{2}+\mathrm{g} \cdot \sigma^{2} .
$$

e) Para a aplicação do teste $\mathrm{F}$ aos componentes de va riância, a nível de parcelas e de subparcelas são testados diretamente com os quadrados médios dos resíduos (a) e (b) respectivamente. A nível de sub-subparcelas faz-se necessāria a adoção das correções nas somas de quadrados, fato es te que dificulta sobremaneira a análise de variância. Suge re-se a utilização do produto g.[Q.M.Resíduo (c)] na estruturação do teste $\mathrm{F}$ para os componentes associados a esse ní vel, como uma forma prática de contornar esse tipo de problema.

f) Os estimadores das variâncias de contrastes envolvendo a unidade perdida, em 12 dos casos possiveis foram: 
f.1) Entre duas médias de tratamentos (A):

$\theta\left(\hat{Y}_{i}\right)=\frac{2}{J K L}\left[E_{a}+f(I-2) E_{c}\right]$

f.2) Entre duas médias de tratamentos (B):

$\hat{\nabla}\left(Q_{k}\right)=\frac{2}{I J L}\left[E_{b}+f(K-2) E_{c}\right]$

f.3) Entre duas médias de tratamentos (C):

$\hat{V}\left(\hat{Y}_{\ell}\right)=\frac{2 E_{C}}{I J K}(I+f \cdot L)$

f.4) Entre duas médias de tratamentos (B) dentro de um mesmo tratamento $\left(A_{i}\right)$ :

$\hat{V}\left(\hat{\mathrm{Y}}_{k / i}\right)=\frac{2}{J L}\left[E_{b}+f(I K-2) E_{c}\right]$

f.5) Entre duas médias de tratamentos (A) dentro de um mesmo tratamento $\left(B_{k}\right)$ :

$\hat{V}\left(\hat{Y}_{i / k}\right)=\frac{2}{J K L}\left[E_{a}+(K-1) E_{b}+f\left(I K^{2}-2 K\right) E_{C}\right]$ 
f.6) Entre duas médias de tratamentos (C) dentro de um mesmo tratamento $\left(A_{i}\right)$ :

$\hat{V}\left(\hat{Y}_{\ell / i}\right)=\frac{2 E_{C}}{J K}(I+f \cdot I L)$

f.7) Entre duas médias de tratamentos (A) dentro de um mesmo tratamento $\left(C_{\ell}\right)$ :

$\hat{V}\left(\hat{Y}_{i / \ell}\right)=\frac{2 E_{a}}{J K L}+\frac{2 E_{c}}{J K L}\left[(L-1)+f\left(I L^{2}-2\right)\right]$

f.8) Entre duas médias de tratamentos (C) dentro de um mesmo tratamento $\left(B_{k}\right)$ :

$\theta\left(\hat{\mathrm{Y}}_{\ell / K}\right)=\frac{2 E_{C}}{I J}(I+\mathrm{f} \cdot \mathrm{KL})$

f.9) Entre duas médias de tratamentos (B) dentro de um mesmo tratamento $\left(C_{\ell}\right)$ :

$\hat{V}\left(\hat{Y}_{k / \ell}\right)=\frac{2 E_{b}}{I J L}+\frac{2 E_{c}}{I J L}\left[(L-1)+f\left(K L^{2}-2\right)\right]$

f.10) Entre duas médias de tratamentos (C) dentro de mesmos niveis de tratamentos $\left(A_{i}\right)$ e $\left(B_{k}\right)$ :

$\theta\left(\hat{Y}_{\ell / i k}\right)=\frac{2 E_{c}}{J}(1+f \cdot I K L)$ 
f.11) Entre duas médias de tratamentos (B) dentro de mesmos niveis de tratamentos $\left(A_{i}\right)$ e $\left(C_{\ell}\right)$ :

$$
\hat{V}\left(\hat{Y}_{k / i \ell}\right)=\frac{2 E_{b}}{J L}+\frac{2 E_{C}}{J L}\left[(L-1)+f\left(I K L^{2}-2\right)\right]
$$

f.12) Entre duas médias de tratamentos (A) dentro de mesmos niveis de tratamentos $\left(B_{k}\right)$ e $\left(C_{\ell}\right)$ :

$$
\hat{V}\left(\hat{Y}_{i / K \ell}\right)=\frac{2 E_{a}}{J K L}+\frac{2(K-1) E_{b}}{J K L}+\frac{2 E_{c}}{J K L}\left[K(L-1)+f\left(I K^{2} L^{2}-2 K\right)\right]
$$

onde:

$\mathrm{E}_{\mathrm{a}}$ é o quadrado médio do resíduo (a);

$E_{b}$ é o quadrado médio do resíduo (b);

$\mathrm{E}_{\mathrm{C}}$ é o quadrado médio do resíduo (c);

$$
f=\frac{1}{2 \operatorname{IK}(J-1)(L-1)}
$$

e ainda:

$$
\begin{aligned}
& I=\text { número de tratamentos primários (A); } \\
& K=\text { número de tratamentos secundārios (B); } \\
& \mathrm{L}=\text { número de tratamentos terciārios (C), e: } \\
& \mathrm{J}=\text { nümero de blocos. }
\end{aligned}
$$


g) Nos casos em que não existir perda de unidade expe rimental o valor de $f$ é zero e, consequentemente, as expressões apresentadas correspondem às publicadas por COCHRAN e COX (1957), LITTLE e HILLS (1977) e outros, para a estimativa da variância de contrastes de médias de tratamentos sem perda de informações. 
ALLAN, F.E. e J. WHISHART, 1930. Method of Estimative the Yield of a Missing Plot in Field Experimental Work. Journal of Agricultural Science. Cambridge, 20: 399-406.

ANDERSON, R.L., 1946. Missing Plot Techniques. Biometrics. Washington, 2: 41-47.

CAMPOS, H., 1964. Estudo sobre a Análise de Experimentos com Parcelas Perdidas. Piracicaba, FSALO/USP, $84 \mathrm{p}$. (Te se de Doutoradol

COCHRAN, W.G. e G. COX, 1957. Experimental Designs. 2a ed. New York, John Wiley \& Sons. 611 p.

HADDAD, M. de L., 1978. Estudo sobre Parcela Perdida em Delineamento de Parcelas Subdivididas. Piracicaba, ESALQ/ USP, 53 p. (Dissertação de Mestrado) 
JOHN, J.A. e M.H. QUENOUILLE, 1977. Experiments: Design and Analysis. London, Charles Griffin \& Company Ltda. 296 p.

LITTLE, T.M. e F.J. HILLS, 1977. Aqricultural Experimentation. New York, John Wiley \& Sons. 350 p.

PIMENTEL GOMES, F., 1976. Curso de Estatistica Experimental. 6 ą ed., Piracicaba, ESALQ/USP. 430 p.

PINHO, S.z., 1973. Observaçöes Perdidas em Delineamentos em Parcelas Subdivididas e Parcelas Sub-subdivididas. Botucatu, UNESP, 173 p. (Tese de Doutorado)

PINHO, S.Z., 1975. Estudo dos Componentes de Variância nos Experimentos em Parcelas Subdivididas com Uma Observação Perdida. Piracicaba, ESALọ/USP, 45 p. (Dissertação de mestrado)

SANCHES, S.F., 1978. Análise de um Experimento em Quadrado Latino com Perda de Parcelas, Linha, Coluna ou Tratamento. Piracicaba, ESALQ/USP, 100 p. (Dissertação de Mestrado)

SANCHES, S.F., 1980. Análise de Experimentos em Parcelas Subdivididas, em blocos casualizados, com Perda de Uma ou Mais Subparcelas ou parcelas. Piracicaba, ESALQ/USP, 119 p. (Tese de Doutorado) 
STEEL, R.G.D. e J.H. TORRIE, 1960. Principles and Procedures of Statistics. New York, McGraw-Hill Book Company Inc. $481 \mathrm{p}$.

TRUITT, J.T. e H.F. SMITH, 1956. Adjustment by Covariance and Consequent Tests of Significance in Split-plot Experiments. Biometrics. Washington, 12: 23-39.

YATES, F., 1933. The Analysis of Replicated Experiments when the Field Results are Incomplete. Empire Jounal of Experimental Agriculture. Oxford, l: 129-142. 
141.

A P ENDICE 
142.

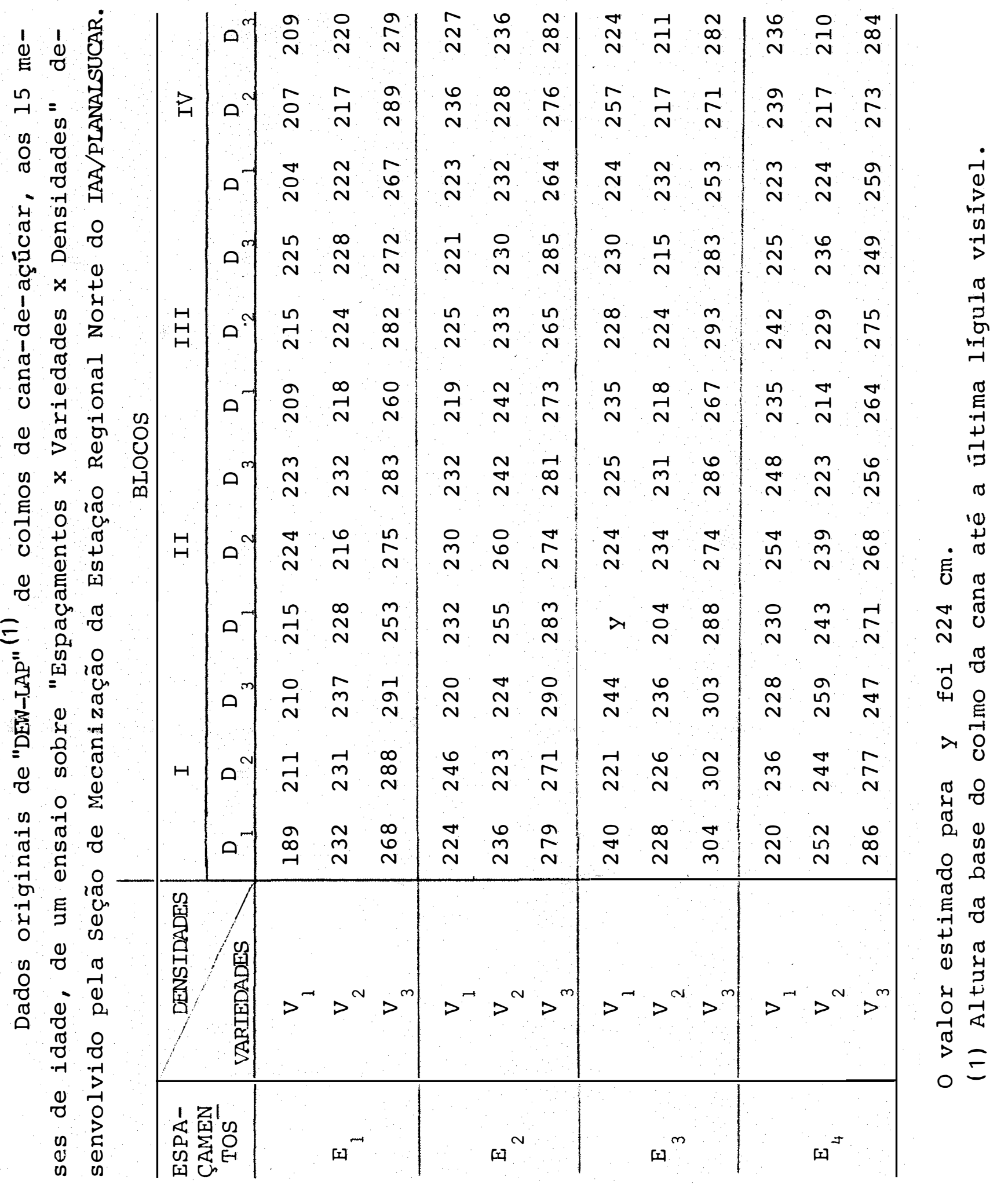

